\title{
Sequential Pd(II)-Pd(0) Catalysis for the Rapid Synthesis of Coumarins.
}

\section{Supporting Information: Table of Contents.}

General procedure for catalytic hydroarylation.

S-2

General procedure for the Suzuki coupling reactions. $\quad$ S-2

General procedure for the hydroarylation/Suzuki coupling. $\quad$ S-2

Spectral characterization of 2a $\quad$ S-3

Spectral characterization of $\mathbf{2 b} \quad$ S-4

Spectral characterization of 2c $\quad$ S-5

Spectral characterization of $\mathbf{2 d} \quad$ S-7

Spectral characterization of $\mathbf{2 e} \quad$ S-8

Spectral characterization of $2 \mathbf{f} \quad$ S-9

Spectral characterization of $\mathbf{2 g} \quad$ S-10

Spectral characterization of $\mathbf{2 h} \quad$ S-11

Spectral characterization of 3a $\quad$ S-12

Spectral characterization of $\mathbf{3 b} \quad$ S-13

Spectral characterization of $\mathbf{3 c} \quad$ S-14

Spectral characterization of 3d $\quad$ S-16

$\begin{array}{ll}\text { Spectral characterization of } \mathbf{3 e} & \text { S-17 }\end{array}$

Spectral characterization of $\mathbf{3 f} \quad$ S-18

$\begin{array}{lr}\text { Spectral characterization of } \mathbf{3 g} & \text { S-19 }\end{array}$

Spectral characterization of $\mathbf{3 h} \quad$ S-20

Spectral characterization of $\mathbf{3 i} \quad$ S-22

Spectral characterization of $\mathbf{3 j} \quad$ S-23

Spectral characterization of $\mathbf{3 k} \quad$ S-24

Spectral characterization of $\mathbf{3 l} \quad$ S-26

$\begin{array}{ll}\text { Spectral characterization of } \mathbf{3 m} & \text { S-27 }\end{array}$

Spectral characterization of $\mathbf{3 n} \quad$ S-28

Spectral characterization of $\mathbf{3 o} \quad$ S-30

Spectral characterization of $\mathbf{3 p} \quad$ S-31

Spectral characterization of $\mathbf{3 q} \quad \mathrm{S}-33$

Spectral characterization of $\mathbf{3 r} \quad \mathrm{S}-34$

Spectral characterization of $\mathbf{3 s} \quad$ S-36

$\begin{array}{ll}\text { Spectral characterization of } \mathbf{3 t} & \text { S-37 }\end{array}$

Spectral characterization of $\mathbf{3 u} \quad$ S-38

$\begin{array}{lr}\text { Spectral characterization of } \mathbf{3 v} & \text { S-39 }\end{array}$

Spectral characterization of $\mathbf{3 w} \quad$ S-41

Spectral characterization of $\mathbf{3} \mathbf{x} \quad \mathrm{S}-42$

Spectral characterization of $\mathbf{3 y} \quad$ S-43 


\section{Experimental.}

Materials. All reagents were used as received. Aryl alkynoates (1) were readily prepared in $60-90 \%$ yield according to the literature method. ${ }^{1}$ NMR spectra were referenced to residual protio solvent signals. Structural assignments are based on ${ }^{1} \mathrm{H},{ }^{13} \mathrm{C}$, DEPT-135, and HMQC spectroscopies.

\section{General procedure for catalytic hydroarylation.}

The aryl alkynoate $1(0.28 \mathrm{mmol}), \operatorname{Pd}(\mathrm{OAc})_{2}(0.014 \mathrm{mmol})$, and 3:1 TFA/DCM (1 mL) were mixed in a reaction tube and stirred at room temperature for 1-6 h. Stirring continued until complete disappearance of the starting material was observed by ${ }^{1} \mathrm{H}$ NMR spectroscopy. The solvent was removed under vacuum and the product was purified by column chromatography on silica gel using $\mathrm{CH}_{2} \mathrm{Cl}_{2}$ as the eluent.

\section{General procedure for the Suzuki coupling reactions.}

Under a $\mathrm{N}_{2}$ atmosphere, THF (1 mL) was added to bromophenylcoumarin $2(0.28 \mathrm{mmol})$, $\mathrm{Pd}(\mathrm{OAc})_{2}(0.0028 \mathrm{mmol})$, arylboronic acid $(0.42 \mathrm{mmol}), \mathrm{KF}(0.84 \mathrm{mmol})$, and 2(dicyclohexylphosphino)biphenyl $(0.056 \mathrm{mmol})$. The reaction mixture was stirred at room temperature for 16-40 h. The solvent was removed under vacuum and the crude mixture was purified by column chromatography on silica gel using $\mathrm{CH}_{2} \mathrm{Cl}_{2}$ as the eluent.

\section{General procedure for the sequential hydroarylation/Suzuki coupling.}

The aryl alkynoate $(0.14 \mathrm{mmol}), \mathrm{Pd}(\mathrm{OAc})_{2}(0.007 \mathrm{mmol})$, and $3: 1 \mathrm{TFA} / \mathrm{DCM}(0.5 \mathrm{~mL})$ were mixed in a reaction tube and stirred at room temperature for 1-6 hrs. The solvent was removed under vacuum and arylboronic acid $(0.21 \mathrm{mmol}), \mathrm{KF}(0.42 \mathrm{mmol})$ and 2(dicyclohexylphosphino)biphenyl $(0.014 \mathrm{mmol})$ were added under an inert atmosphere $\left(\mathrm{N}_{2}\right.$ or $\left.\mathrm{Ar}\right)$. THF $(1 \mathrm{~mL})$ was added and the reaction mixture was heated at $50{ }^{\circ} \mathrm{C}$ for $16 \mathrm{~h}$. The solvent was removed under vacuum and the crude mixture was purified by column chromatography on silica gel using $\mathrm{CH}_{2} \mathrm{Cl}_{2}$ as the eluent. 


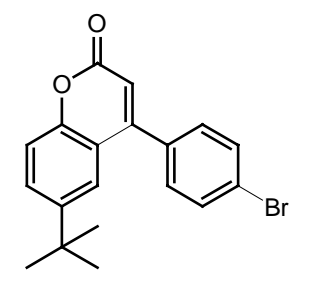

4-(4-Bromophenyl)-6-tert-butyl-2H-chromen-2-one (2a). ${ }^{1} \mathrm{H}$ NMR $\left(\mathrm{CDCl}_{3}\right): \delta 7.67$ (d, $2 \mathrm{H}, P h \mathrm{Br}, J=8.8 \mathrm{~Hz}) ; 7.60\left(\mathrm{dd}, 1 \mathrm{H},{ }^{t} \mathrm{Bu} P h, J=8.8\right.$ and $\left.2.3 \mathrm{~Hz}\right) ; 7.41\left(\mathrm{~d}, 1 \mathrm{H},{ }^{t} \mathrm{Bu} P h, J=\right.$ $2.3 \mathrm{~Hz}$ ); 7.35 (d, 2H, $P h \mathrm{Br}, J=8.8 \mathrm{~Hz}) ; 7.33\left(\mathrm{~d}, 1 \mathrm{H},{ }^{t} \mathrm{Bu} P h, J=8.8 \mathrm{~Hz}\right) ; 6.33(\mathrm{~s}, 1 \mathrm{H}$, CHCO); 1.26 (s, 9H, $\left.{ }^{t} \mathrm{Bu}\right) .{ }^{13} \mathrm{C}\left\{{ }^{1} \mathrm{H}\right\}$ NMR: $\delta 160.6(\mathrm{CO}), \mathrm{Ph}$ ring and $\mathrm{C}=\mathrm{C}(154.6,152.1$, 147.3, 134.1, 132.1, 129.9, 129.7, 124.1, 122.7, 117.7, 116.9, 115.1), ${ }^{t} \mathrm{Bu}(34.5,31.2)$. $v_{\max }(\mathrm{NaCl}) / \mathrm{cm}^{-1}: 3055,1722,1616,1570,1489,1256,1184,1128,1013$. HR-MS: $\mathrm{C}_{19} \mathrm{H}_{18} \mathrm{BrO}_{2}$ Calcd. 357.0490 (M+1); found: 357.0498 .

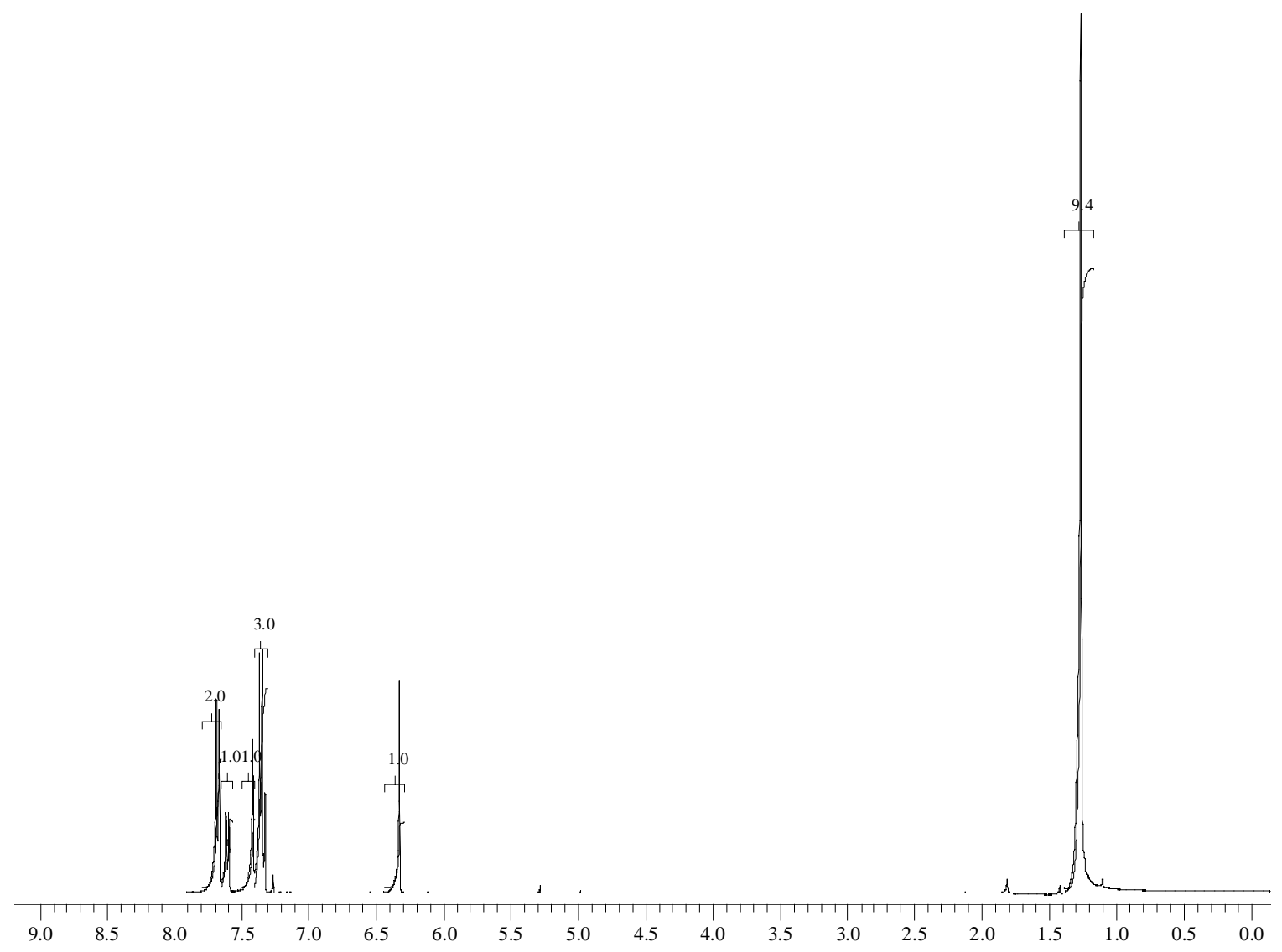



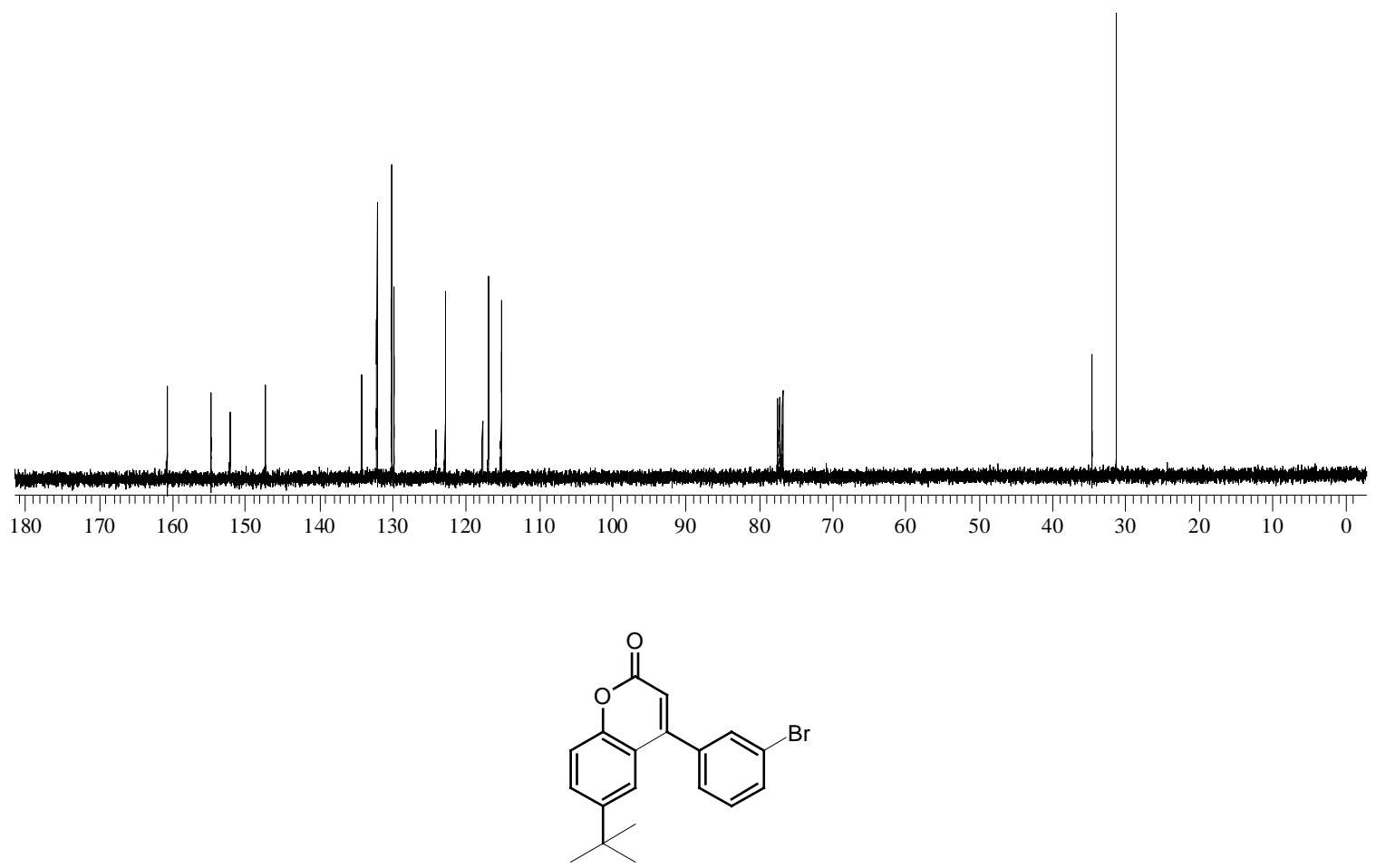

4-(3-Bromophenyl)-6-tert-butyl-2H-chromen-2-one (2b). ${ }^{1} \mathrm{H} \mathrm{NMR}\left(\mathrm{CDCl}_{3}\right): \delta 7.68(\mathrm{dt}$, $1 \mathrm{H}, P h \mathrm{Br}, J=7.0$ and $2.0 \mathrm{~Hz}) ; 7.64(\mathrm{~m}, 1 \mathrm{H}, P h \mathrm{Br}) ; 7.62\left(\mathrm{dd}, 1 \mathrm{H},{ }^{t} \mathrm{Bu} P h, J=8.8\right.$ and 2.3 $\mathrm{Hz}) ; 7.43(\mathrm{t}, 1 \mathrm{H}, P h \mathrm{Br}, J=7.6 \mathrm{~Hz}) ; 7.39-7.42\left(\mathrm{~m}, 2 \mathrm{H},{ }^{t} \mathrm{Bu} P h\right.$ and $\left.P h \mathrm{Br}\right) ; 7.36(\mathrm{~d}, 1 \mathrm{H}$, $\left.{ }^{t} \mathrm{Bu} P h, J=8.8 \mathrm{~Hz}\right) ; 6.36(\mathrm{~s}, 1 \mathrm{H}, \mathrm{CHCO}) ; 1.28\left(\mathrm{~s}, 9 \mathrm{H},{ }^{t} \mathrm{Bu}\right) .{ }^{13} \mathrm{C}\left\{{ }^{1} \mathrm{H}\right\} \mathrm{NMR}: \delta 160.6(\mathrm{CO})$, $\mathrm{Ph}$ ring and $\mathrm{C}=\mathrm{C}(154.1,152.1,147.3,132.6,131.3,130.3,129.8,127.0,122.9,122.7$, 117.7, 116.9, 115.3), ${ }^{t} \mathrm{Bu}(34.6,31.2) . v_{\max }(\mathrm{NaCl}) / \mathrm{cm}^{-1}: 3055,1724,1616,1568,1558$, 1489, 1473, 1369, 1186, 1130. HR-MS: $\mathrm{C}_{19} \mathrm{H}_{18} \mathrm{BrO}_{2}$ Calcd. 357.0490 (M+1); found: 357.0488 . 


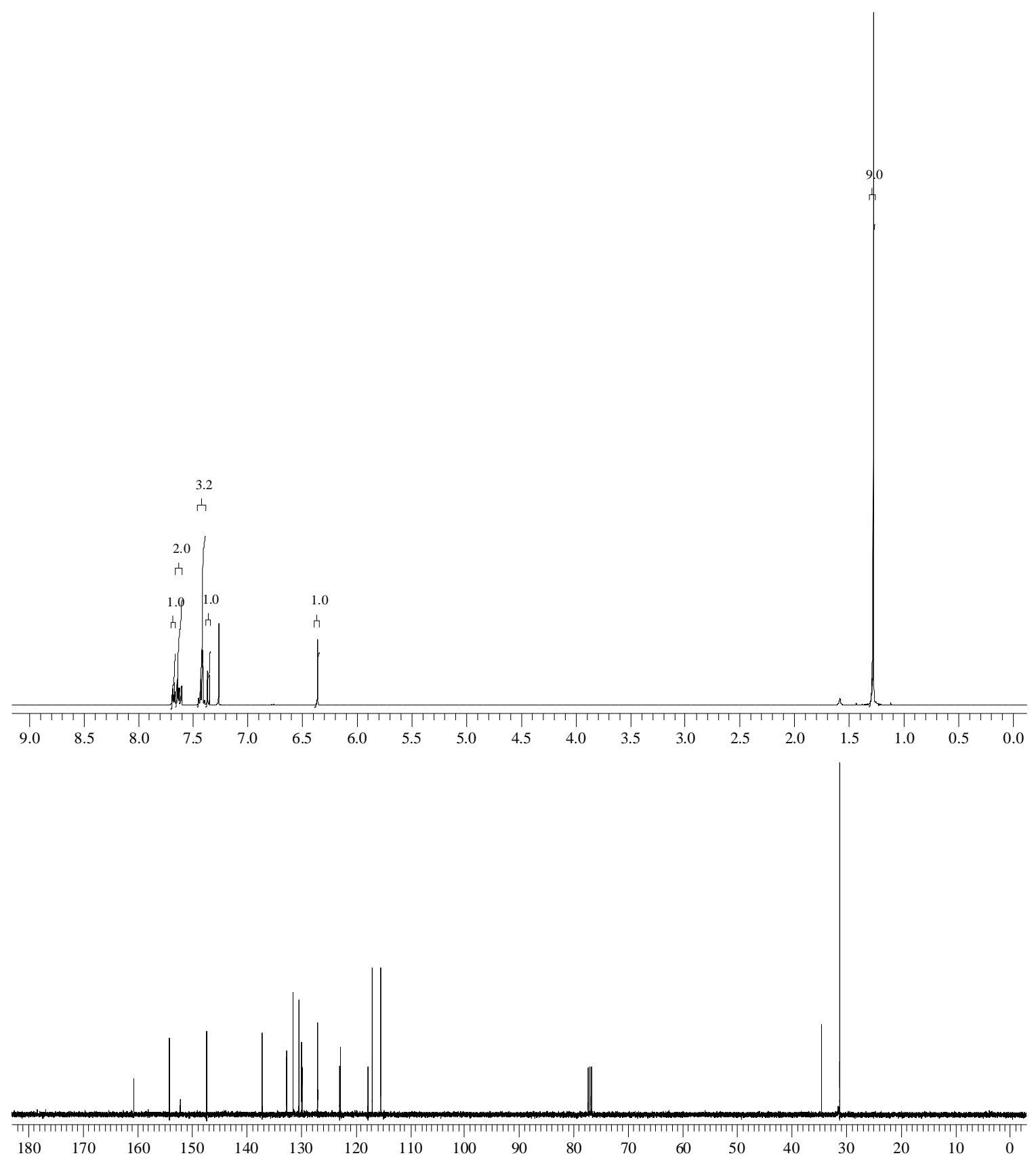

4-(2-Bromophenyl)-6-tert-butyl-2H-chromen-2-one (2c). ${ }^{1} \mathrm{H} \mathrm{NMR}\left(\mathrm{CDCl}_{3}\right): \delta 7.75(\mathrm{~d}$, $1 \mathrm{H}, P h \mathrm{Br}, J=7.9 \mathrm{~Hz}) ; 7.59\left(\mathrm{dd}, 1 \mathrm{H},{ }^{t} \mathrm{Bu} P h, J=8.5\right.$ and $\left.2.3 \mathrm{~Hz}\right) ; 7.48(\mathrm{t}, 1 \mathrm{H}, P h \mathrm{Br}, J=$ $7.6 \mathrm{~Hz}) ; 7.40$ (d, 1H, PhBr, $J=7.6 \mathrm{~Hz}) ; 7.30-7.35$ (m, 2H, PhBr, $1 \mathrm{H} ;{ }^{t} \mathrm{Bu} P h, 1 \mathrm{H} ;$ ); 7.04 
(d, $\left.1 \mathrm{H},{ }^{t} \mathrm{Bu} P h, J=2.3 \mathrm{~Hz}\right) ; 6.33$ (s, $\left.1 \mathrm{H}, \mathrm{CHCO}\right) ; 1.26$ (s, 9H, $\left.{ }^{t} \mathrm{Bu}\right) \cdot{ }^{13} \mathrm{C}\left\{{ }^{1} \mathrm{H}\right\}$ NMR: $\delta$ $160.9(\mathrm{CO}), \mathrm{Ph}$ ring and $\mathrm{C}=\mathrm{C}(155.1,151.7,147.2,136.2,133.2,130.7,130.2,129.5$, 127.7, 123.2, 121.9, 117.8, 116.6, 116.1), ${ }^{t} \mathrm{Bu}(34.4,31.2) . v_{\max }(\mathrm{NaCl}) / \mathrm{cm}^{-1}: 3055,1722$, 1615, 1570, 1472, 1369, 1184, 1132, 1030. HR-MS: $\mathrm{C}_{18} \mathrm{H}_{18} \mathrm{O}_{2} \mathrm{Br}$ Calcd. $357.0490(\mathrm{M}+1)$; found: 357.0482 .

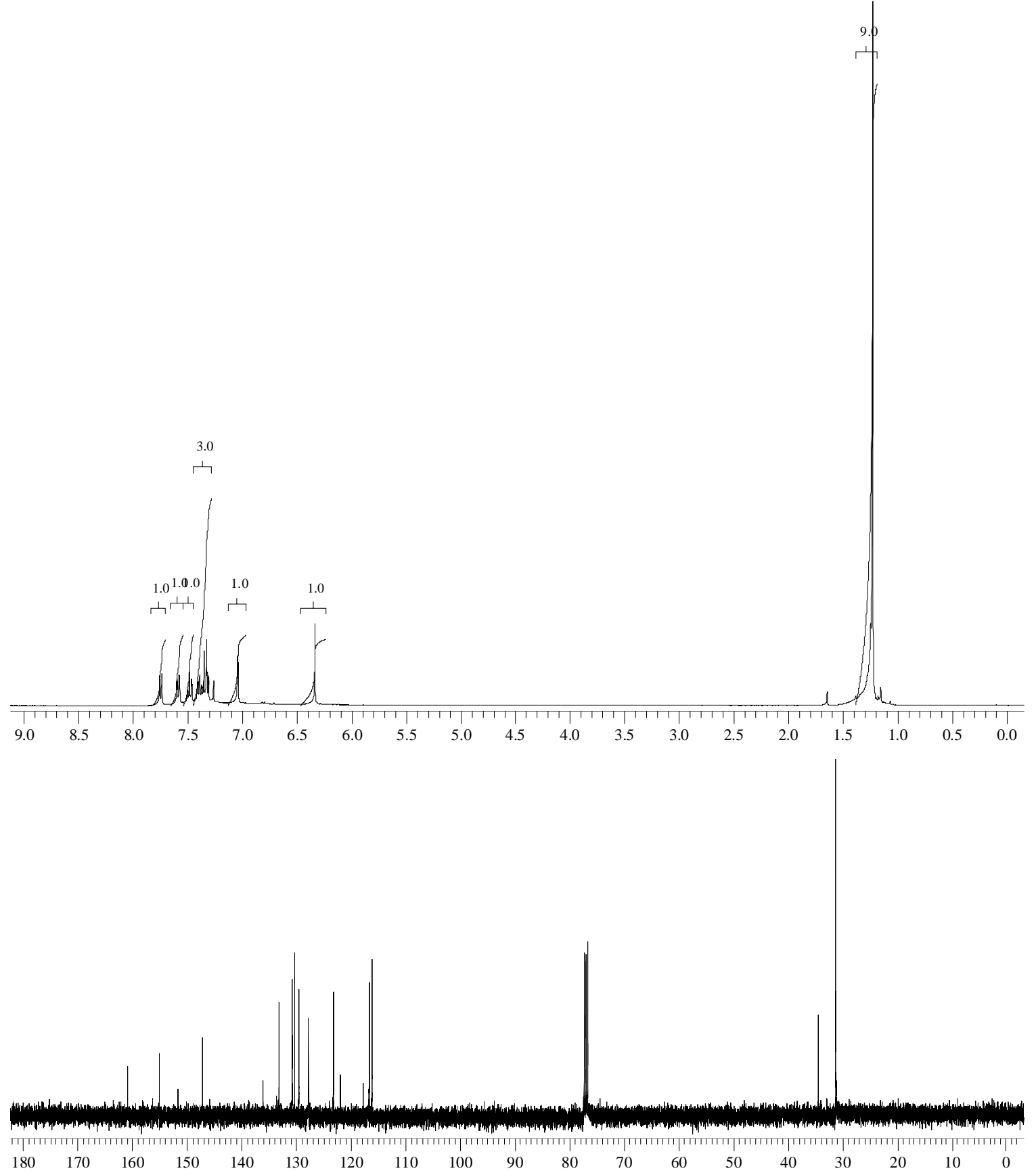




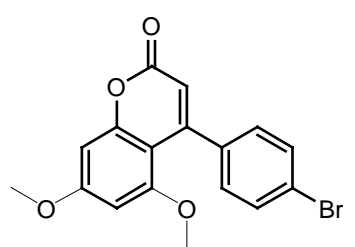

4-(4-Bromophenyl)-5,7-dimethoxy-2H-chromen-2-one (2d). ${ }^{1} \mathrm{H} \mathrm{NMR}\left(\mathrm{CDCl}_{3}\right): \delta 7.49$ $\left(\mathrm{d}, 2 \mathrm{H}, \mathrm{PhBr}, J=8.2 \mathrm{~Hz}\right.$ ); 7.13 (d, 2H, $\mathrm{PhBr}, J=8.2 \mathrm{~Hz}$ ); 6.49 (d, $1 \mathrm{H}, P h \mathrm{OCH}_{3}, J=2.0$ $\mathrm{Hz}) ; 6.22\left(\mathrm{~d}, 1 \mathrm{H}, \mathrm{PhOCH}_{3}, J=2.4 \mathrm{~Hz}\right) ; 5.94$ (s, 1H, CHCO); 3.85 (s, 3H, $\left.\mathrm{PhOCH}_{3}\right) ; 3.45$ (s, $\left.3 \mathrm{H}, \mathrm{PhOCH} \mathrm{H}_{3}\right){ }^{13} \mathrm{C}\left\{{ }^{1} \mathrm{H}\right\}$ NMR: $\delta 160.5(\mathrm{CO})$, Ph ring and $\mathrm{C}=\mathrm{C}(163.5,158.0,157.1$, 154.3, 138.6, 130.5, 128.8, $122.0112 .6,103.0,95.6,93.5), \mathrm{PhOCH}_{3}(55.7,55.3) . v_{\max }$ $(\mathrm{NaCl}) / \mathrm{cm}^{-1}:$ 3057, 1718, 1614, 1599, 1497, 1465, 1354, 1207, 1159, 1113, 1053, 1012. HR-MS: $\mathrm{C}_{17} \mathrm{H}_{14} \mathrm{O}_{4} \mathrm{Br}$ Calcd. 361.0075 (M+1); found: 361.0084 .

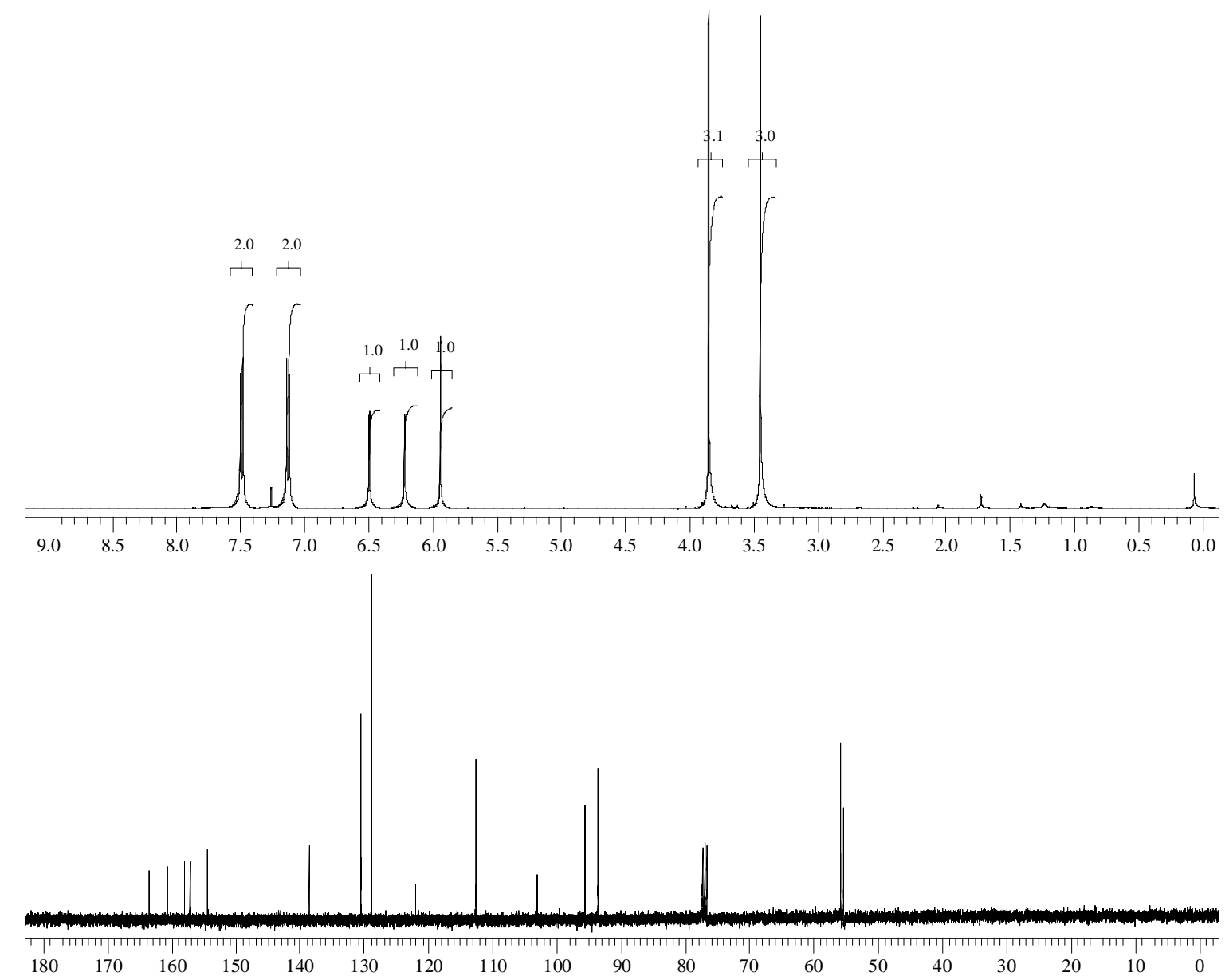




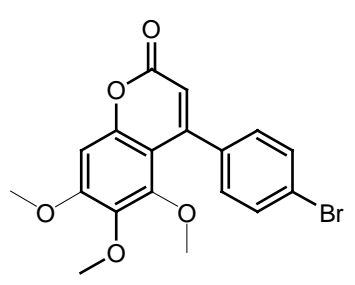

4-(4-Bromophenyl)-5,6,7-trimethoxy-2H-chromen-2-one (2e). ${ }^{1} \mathrm{H}$ NMR $\left(\mathrm{CDCl}_{3}\right)$ : $\delta$ 7.55 (d, 2H, $P h \mathrm{Br}, J=8.5 \mathrm{~Hz}) ; 7.21(\mathrm{~d}, 2 \mathrm{H}, P h \mathrm{Br}, J=8.5 \mathrm{~Hz}) ; 6.72\left(\mathrm{~s}, 1 \mathrm{H}, P h \mathrm{OCH}_{3}\right)$; 6.04 (s, 1H, CHCO); 3.94 (s, 3H, $\mathrm{PhOCH}_{3}$ ); 3.39 (s, 3H, $\left.\mathrm{PhOCH}_{3}\right)$; 3.30 (s, 3H, $\mathrm{PhOCH}_{3}$ ). ${ }^{13} \mathrm{C}\left\{{ }^{1} \mathrm{H}\right\}$ NMR: $\delta 160.3(\mathrm{CO}), \mathrm{Ph}$ ring and $\mathrm{C}=\mathrm{C}(157.0,154.0,151.6,150.8,139.3,137.8$, 130.6, 128.9, 122.1, 114.0, 106.7, 96.2), $\mathrm{PhOCH}_{3}(61.02,60.96,56.2) . v_{\max }(\mathrm{NaCl}) / \mathrm{cm}^{-1}$ : 3058, 1722, 1605, 1549, 1489, 1458, 1410, 1364, 1200, 1132, 1101, 1036. HR-MS: $\mathrm{C}_{18} \mathrm{H}_{16} \mathrm{O}_{5} \mathrm{Br}$ Calcd. $391.0181(\mathrm{M}+1)$; found: 391.0176.

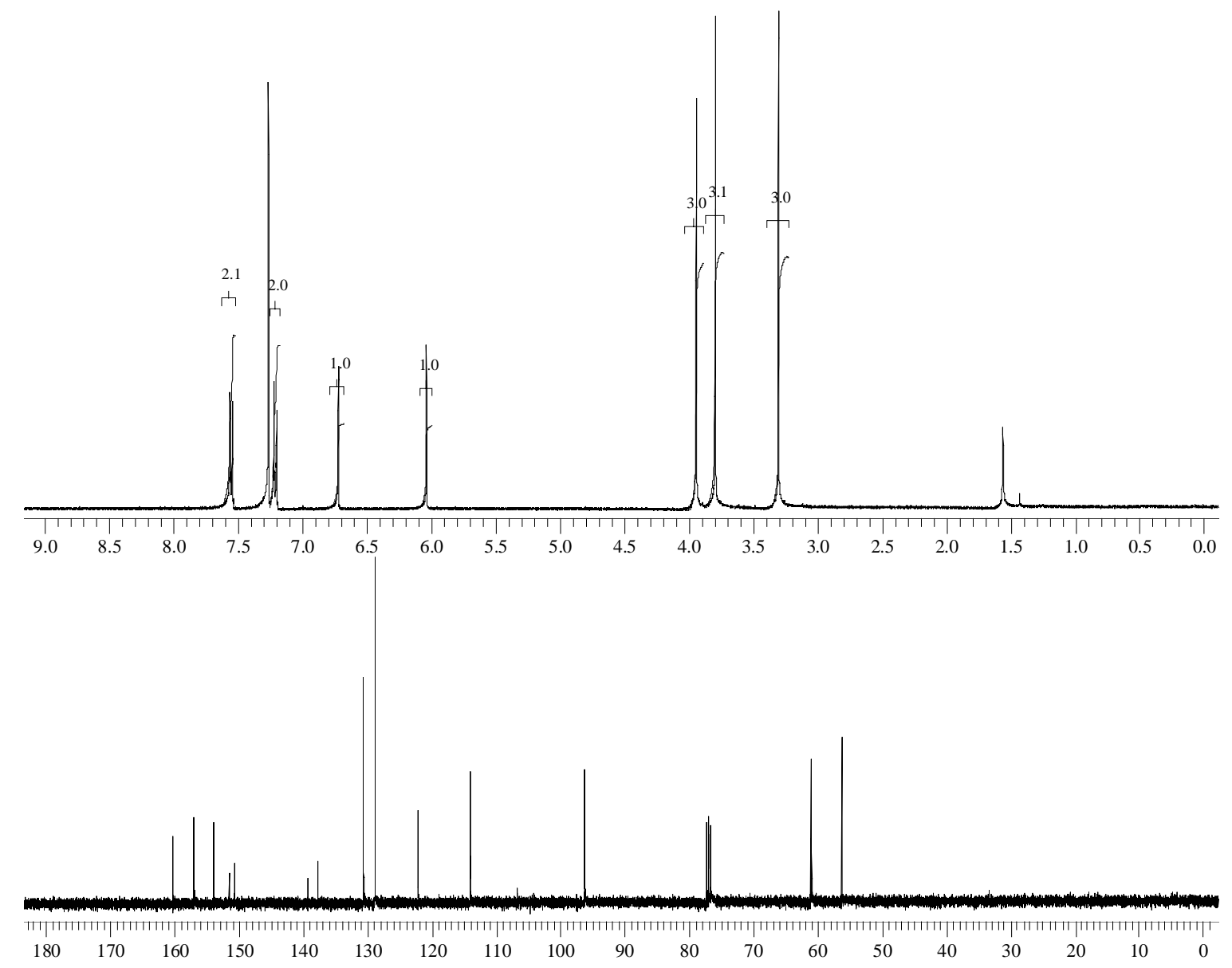




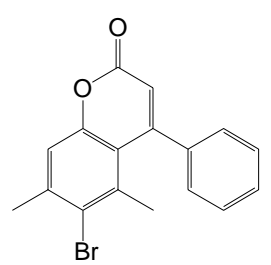

6-Bromo-5,7-dimethyl-4-phenyl-2H-chromen-2-one (2f). ${ }^{2} \mathrm{H}$ NMR $\left(\mathrm{CDCl}_{3}\right): \delta$ 7.45$7.47(\mathrm{~m}, 3 \mathrm{H}, P h \mathrm{C}=\mathrm{CH}) ; 7.27-7.30(\mathrm{~m}, 2 \mathrm{H}, P h \mathrm{C}=\mathrm{CH}) ; 7.20\left(\mathrm{~s}, 1 \mathrm{H}, P h \mathrm{CH}_{3}\right) ; 7.05(\mathrm{dd}, 2 \mathrm{H}$, PhPhO, $J=6.7$ and $1.5 \mathrm{~Hz}) ; 6.26$ (s, $1 \mathrm{H}, \mathrm{CHCO}) ; 2.51$ (s, 3H, $\mathrm{PhCH}_{3}$ ); 1.95 (s, 3H, $\left.\mathrm{PhCH}_{3}\right) .{ }^{13} \mathrm{C}\left\{{ }^{1} \mathrm{H}\right\}$ NMR: $\delta 159.9(\mathrm{CO}), \mathrm{Ph}$ ring and $\mathrm{C}=\mathrm{C}(156.1,153.6,142.7,139.5$, 136.8, 129.1, 128.9, 127.1, 125.8, 117.41, 117.39, 116.9), $\mathrm{PhCH}_{3}(24.8,24.7)$.
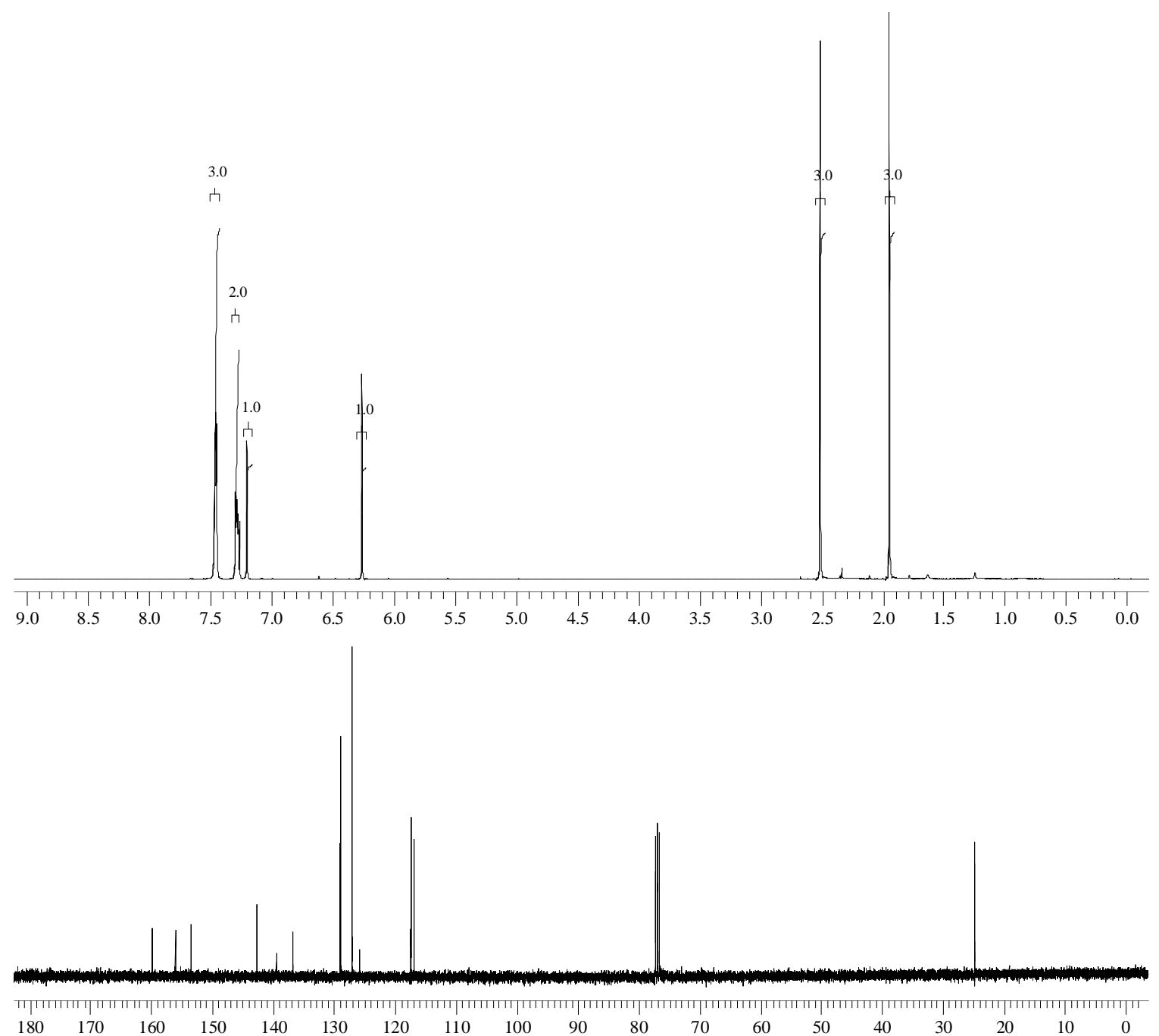
<smiles>CCc1cc(=O)oc2cc(C)c(Br)c(C)c12</smiles>

6-Bromo-5,7-dimethyl-4-pentyl-2H-chromen-2-one (2g). ${ }^{1} \mathrm{H}$ NMR $\left(\mathrm{CDCl}_{3}\right): \delta 7.11$ (s, $1 \mathrm{H}, \mathrm{Ph}) ; 6.24$ (s, 1H, CHCO); 2.89 (t, 2H, CH=CCH $\left.\mathrm{CH}_{2}=7.9 \mathrm{~Hz}\right) ; 2.79$ (s, 3H, $\left.\mathrm{PhCH}_{3}\right)$; 2.47 (s, 3H, $\left.\mathrm{PhCH}_{3}\right) ; 1.58-1.65\left(\mathrm{~m}, 2 \mathrm{H}, \mathrm{CH}=\mathrm{CCH}_{2} \mathrm{CH}_{2}\right) ; 1.31-1.44$ (m, 4H, $\left.\mathrm{CH}_{2} \mathrm{CH}_{2} \mathrm{CH}_{3}\right)$; $0.91\left(\mathrm{t}, 3 \mathrm{H}, \mathrm{CH}_{2} \mathrm{CH}_{3}, J=7.0 \mathrm{~Hz}\right) .{ }^{13} \mathrm{C}\left\{{ }^{1} \mathrm{H}\right\} \mathrm{NMR}: \delta 160.2(\mathrm{CO}), \mathrm{Ph}$ ring and $\mathrm{C}=\mathrm{C}(157.7$, 153.4, 142.1, 135.6, 125.9, 118.2, 117.4, 115.7), $36.7\left(\mathrm{CCH}_{2}\right), 31.5\left(\mathrm{CCH}_{2} \mathrm{CH}_{2}\right), 29.4$ $\left(\mathrm{CH}_{2} \mathrm{CH}_{2} \mathrm{CH}_{3}\right), 25.0\left(\mathrm{CH}_{2} \mathrm{CH}_{3}\right), 23.6\left(\mathrm{PhCH}_{3}\right), 22.4\left(\mathrm{PhCH}_{3}\right), 13.9\left(\mathrm{CH}_{2} \mathrm{CH}_{3}\right) . \quad v_{\max }$ $(\mathrm{NaCl}) / \mathrm{cm}^{-1}: 3058,1724,1599,1535,1448,1427,1380,1356,1230,1178,1105,1041$. HR-MS: $\mathrm{C}_{16} \mathrm{H}_{20} \mathrm{O}_{2} \mathrm{Br}$ Calcd. $323.0647(\mathrm{M}+\mathrm{H})$; found: 323.0647.
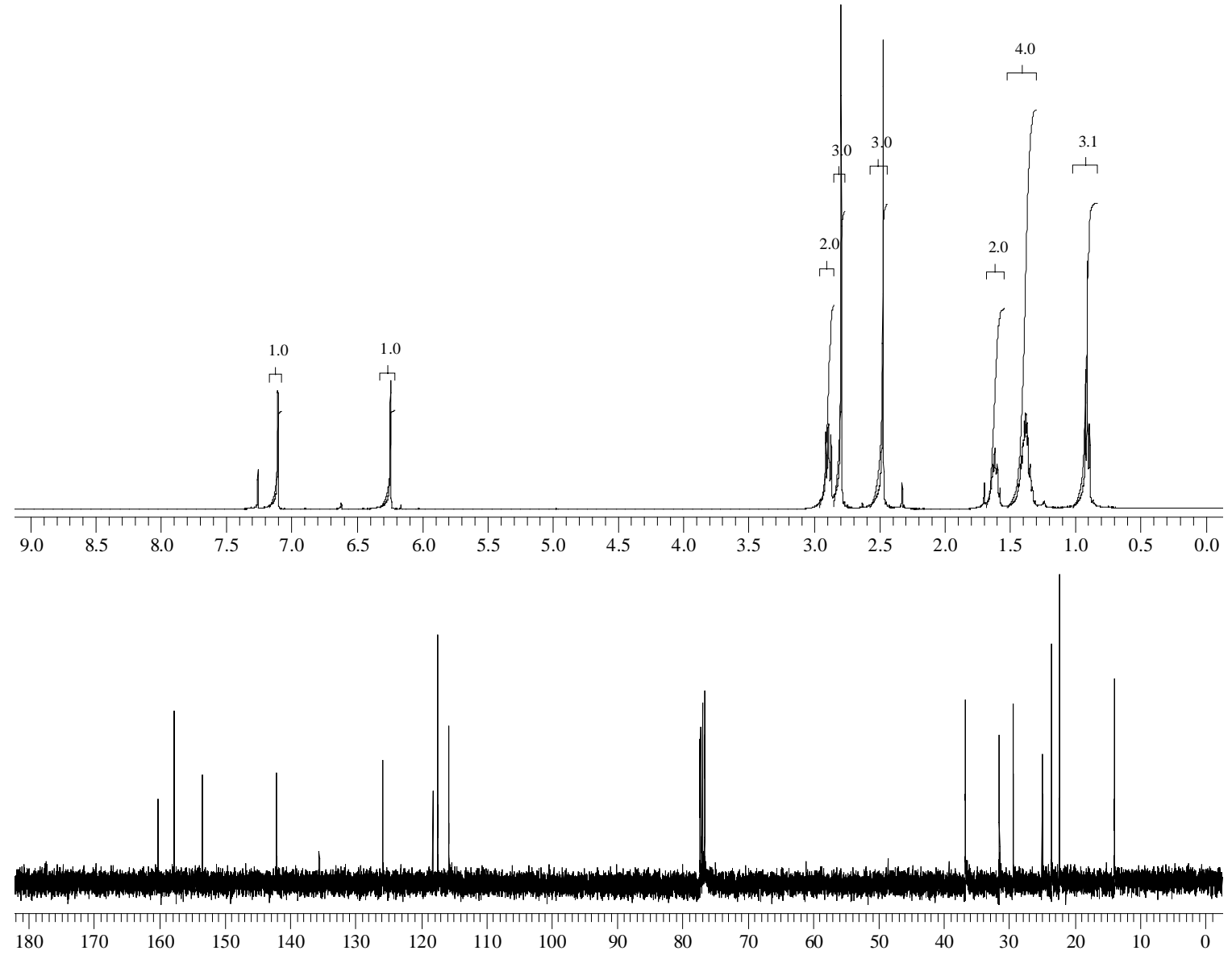


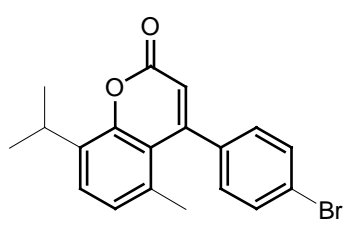

4-(4-Bromophenyl)-8-isopropyl-5-methyl-2H-chromen-2-one (2h). ${ }^{1} \mathrm{H}$ NMR $\left(\mathrm{CDCl}_{3}\right)$ : $\delta 7.59$ (d, 2H, $P h \mathrm{Br}, J=8.2 \mathrm{~Hz}) ; 7.37$ (d, 1H, $\mathrm{PhO}, J=7.6 \mathrm{~Hz}) ; 7.18$ (d, 2H, $P h \mathrm{Br}, J=$ $8.2 \mathrm{~Hz}$ ); 6.99 (d, 1H, PhO, J = 7.6 Hz); 6.22 (s, 1H, CHCO); 3.62-3.69 (m, 1H, $\mathrm{CHCH}_{3}$ ); 1.82 (s, 3H, $\left.\mathrm{PhCH}_{3}\right) ; 1.30$ (d, 6H, $\left.\mathrm{CHCH}_{3}, J=6.7 \mathrm{~Hz}\right) .{ }^{13} \mathrm{C}\left\{{ }^{1} \mathrm{H}\right\}$ NMR: $\delta 160.0(\mathrm{CO}), \mathrm{Ph}$ ring and $\mathrm{C}=\mathrm{C}(155.9,152.1,138.7,134.9,134.3,131.8,129.0,128.7,128.1,123.0,117.3$, 117.1), $\mathrm{CHCH}_{3}(26.5,22.7), 23.7\left(\mathrm{PhCH}_{3}\right) . v_{\max }(\mathrm{NaCl}) / \mathrm{cm}^{-1}: 3055,1720,1578,1489$, 1447, 1416, 1196, 1051, 1015. HR-MS: $\mathrm{C}_{19} \mathrm{H}_{18} \mathrm{O}_{2} \mathrm{Br}$ Calcd. $357.0490(\mathrm{M}+1)$; found: 357.0502 .

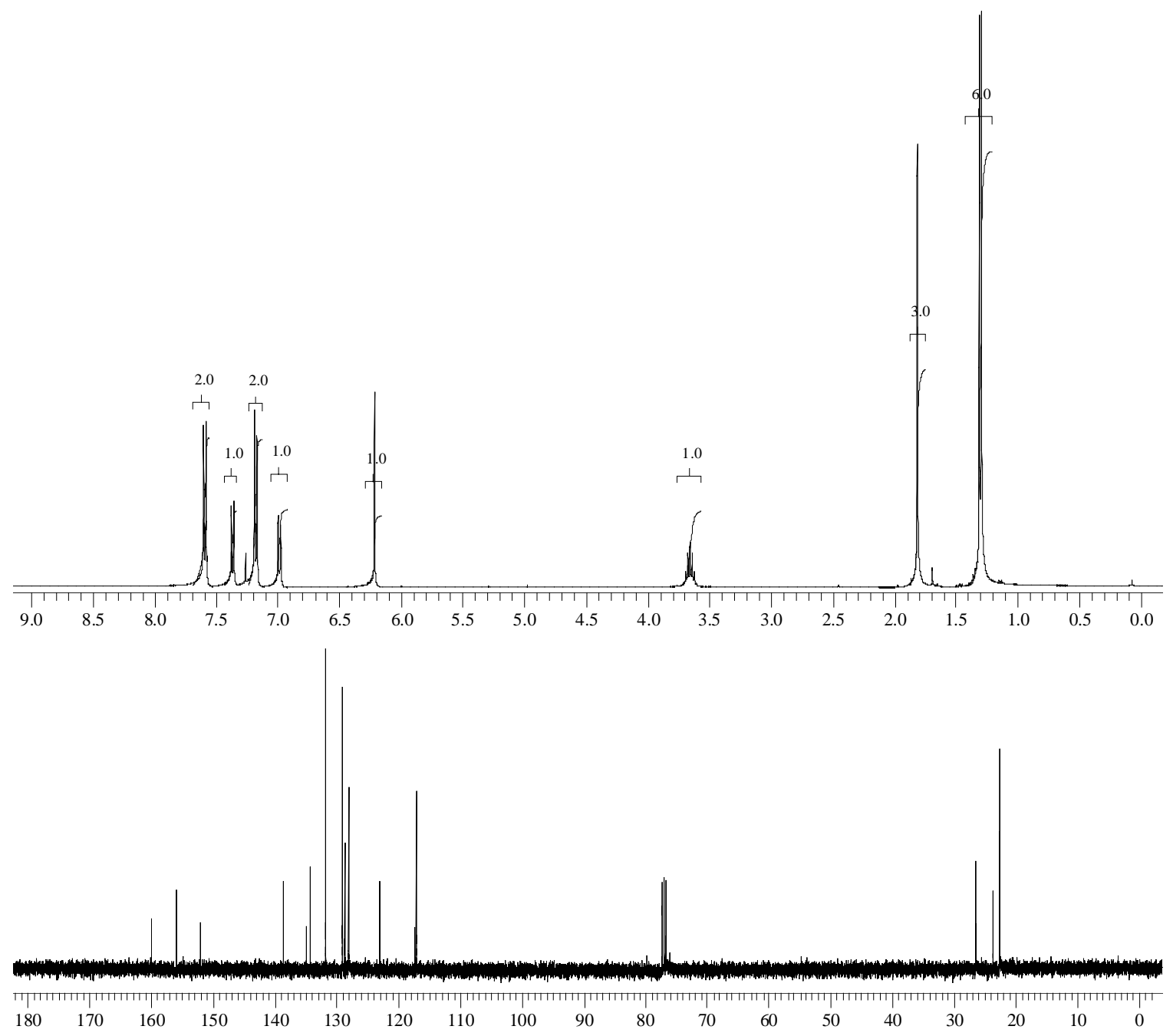




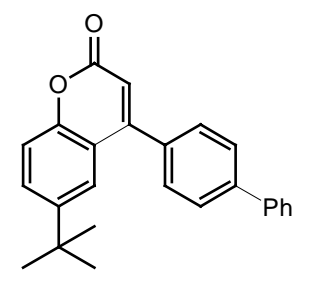

6-tert-Butyl-4-(4-biphenyl)-2H-chromen-2-one (3a). ${ }^{1} \mathrm{H} \mathrm{NMR}\left(\mathrm{CDCl}_{3}\right): \delta 7.77(\mathrm{~d}, 2 \mathrm{H}$, $\mathrm{PhPhC}, J=8.2 \mathrm{~Hz}) ; 7.69$ (d, 2H, $P h \mathrm{PhC}, J=7.3 \mathrm{~Hz}) ; 7.62\left(\mathrm{dd}, 1 \mathrm{H},{ }^{t} \mathrm{Bu} P h, J=8.8\right.$ and $2.3 \mathrm{~Hz}) ; 7.58\left(\mathrm{~s}, 1 \mathrm{H},{ }^{t} \mathrm{Bu} P h\right) ; 7.57(\mathrm{~d}, 2 \mathrm{H}, \mathrm{Ph} P h \mathrm{C}, J=8.2 \mathrm{~Hz}) ; 7.51$ (t, 2H,PhPhC, $J=$ $7.6 \mathrm{~Hz}) ; 7.42$ (t, $1 \mathrm{H}, P h \mathrm{PhC}, J=7.6 \mathrm{~Hz}) ; 7.37$ (d, $\left.1 \mathrm{H},{ }^{t} \mathrm{Bu} P h, J=8.8 \mathrm{~Hz}\right) ; 6.42(\mathrm{~s}, 1 \mathrm{H}$, $\mathrm{CHCO}) ; 1.29\left(\mathrm{~s}, 9 \mathrm{H},{ }^{t} \mathrm{Bu}\right) .{ }^{13} \mathrm{C}\left\{{ }^{1} \mathrm{H}\right\} \mathrm{NMR}: \delta 161.1(\mathrm{CO}), \mathrm{Ph}$ ring and $\mathrm{C}=\mathrm{C}(155.6,152.2$, 147.2 , 142.5, 139.9, 134.1, 129.5, 128.95, 128.94, 127.9, 127.4, 127.1, 123.1, 118.1, 116.9, 114.9), ${ }^{t} \mathrm{Bu}(34.6,31.3) . v_{\max }(\mathrm{NaCl}) / \mathrm{cm}^{-1}: 3055,1720,1614,1570,1489,1369$, 1256, 1186, 1130. HR-MS: $\mathrm{C}_{25} \mathrm{H}_{23} \mathrm{O}_{2}$ Calcd. 355.1698 (M+1); found: 355.1709.

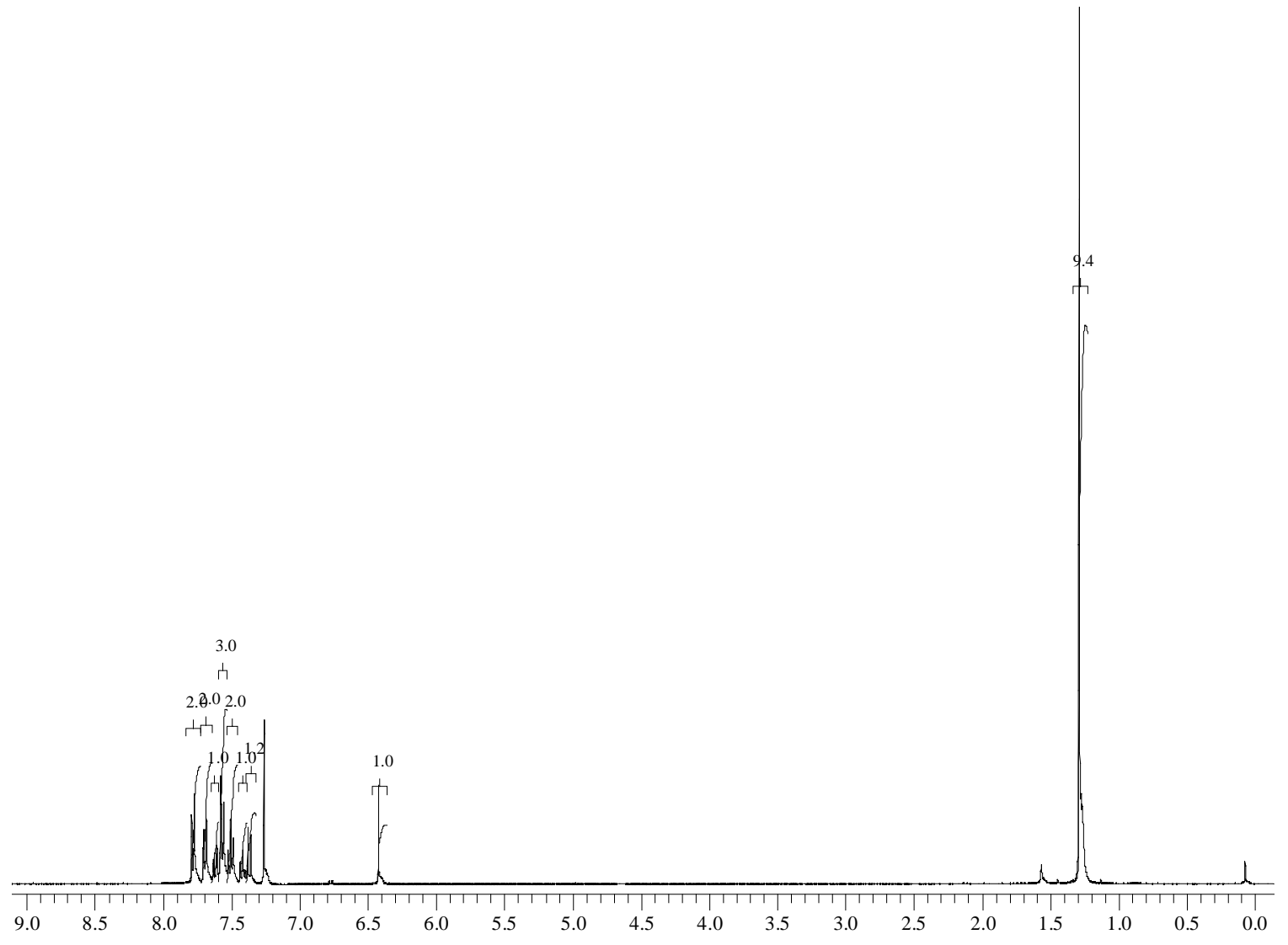



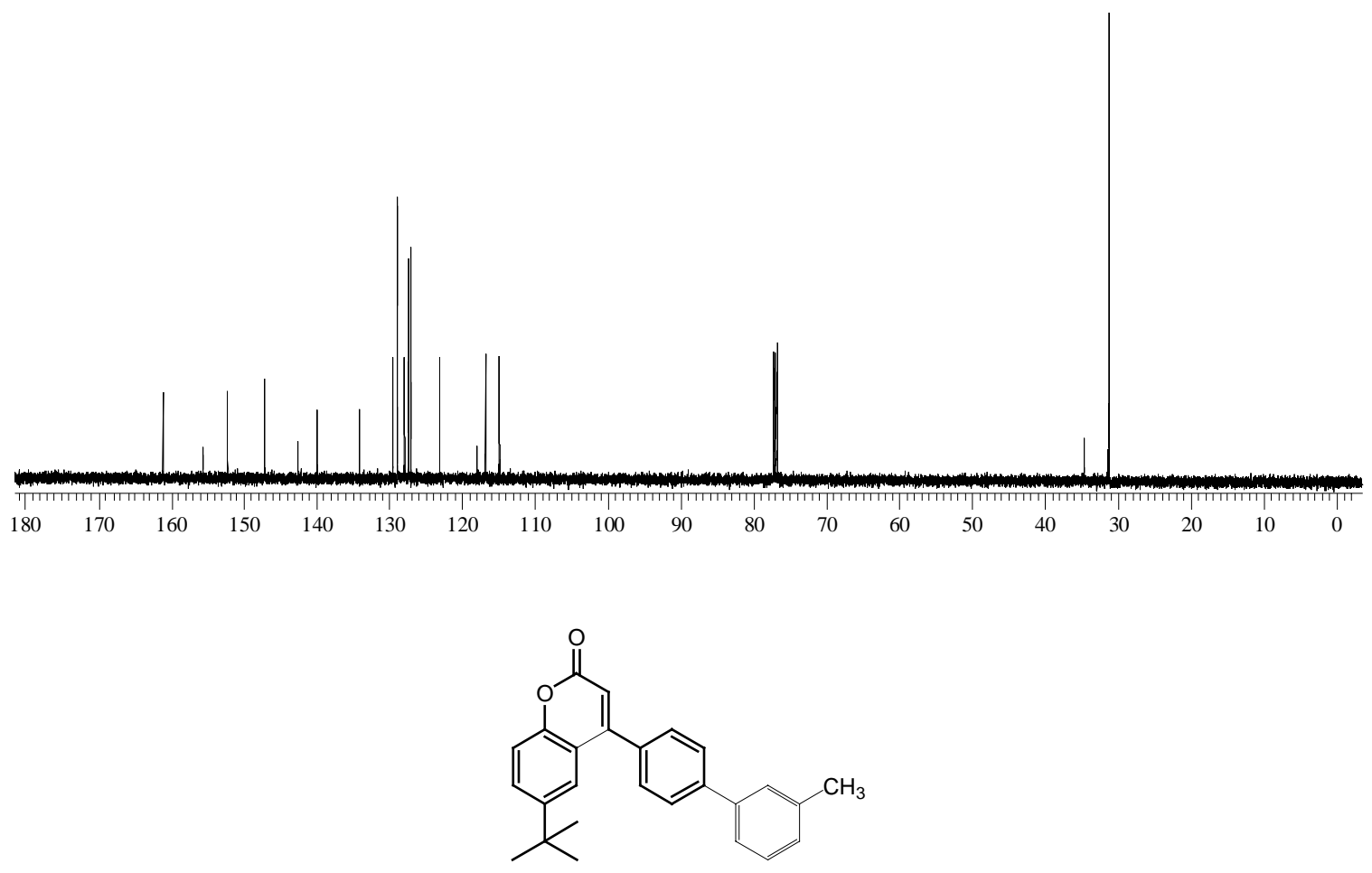

6-tert-Butyl-4-(4-m-tolylphenyl)-2H-chromen-2-one (3b). ${ }^{1} \mathrm{H} \mathrm{NMR}\left(\mathrm{CDCl}_{3}\right): \delta 7.77(\mathrm{~d}$, $\left.2 \mathrm{H}, P h \mathrm{PhCH}_{3}, J=8.5 \mathrm{~Hz}\right) ; 7.62\left(\mathrm{dd}, 1 \mathrm{H},{ }^{t} \mathrm{Bu} P h, J=8.8\right.$ and $\left.2.3 \mathrm{~Hz}\right) ; 7.58\left(\mathrm{~d}, 1 \mathrm{H},{ }^{t} \mathrm{Bu} P h\right.$, $J=2.3 \mathrm{~Hz}) ; 7.56\left(\mathrm{~d}, 2 \mathrm{H}, P h \mathrm{PhCH}_{3}, J=8.5 \mathrm{~Hz}\right) ; 7.51$ (s, $\left.1 \mathrm{H}, P h \mathrm{CH}_{3}\right) ; 7.49$ (d, br, $1 \mathrm{H}$, $\left.P h \mathrm{CH}_{3}, J=7.6 \mathrm{~Hz}\right) ; 7.39\left(\mathrm{t}, 1 \mathrm{H}, P h \mathrm{CH}_{3}, J=7.3 \mathrm{~Hz}\right) ; 7.37\left(\mathrm{~d}, 1 \mathrm{H},{ }^{t} \mathrm{Bu} P h, J=8.8 \mathrm{~Hz}\right)$; 7.24 (d, 1H, $\left.P h \mathrm{CH}_{3}, J=7.3 \mathrm{~Hz}\right) ; 6.42$ (s, 1H, CHCO); 2.46 (s, 3H, PhCH $) ; 1.29$ (s, 9H, $\left.{ }^{t} \mathrm{Bu}\right) .{ }^{13} \mathrm{C}\left\{{ }^{1} \mathrm{H}\right\} \mathrm{NMR}: \delta 161.1(\mathrm{CO}), \mathrm{Ph}$ ring and $\mathrm{C}=\mathrm{C}(155.6,152.2,147.2,142.7,139.9$, 138.6, 134.1, 129.53, 129.52, 128.91, 128.87, 128.7, 127.9, 124.2, 123.2, 118.1, 116.9, 114.9), ${ }^{t} \mathrm{Bu}(34.6,31.3), 21.5\left(\mathrm{PhCH}_{3}\right) . v_{\max }(\mathrm{NaCl}) / \mathrm{cm}^{-1}: 1062,1720,1614,1570,1486$, 1371, 1186, 1130. HR-MS: $\mathrm{C}_{26} \mathrm{H}_{25} \mathrm{O}_{2}$ Calcd. 369.1855 (M+1); found: 369.1846. 


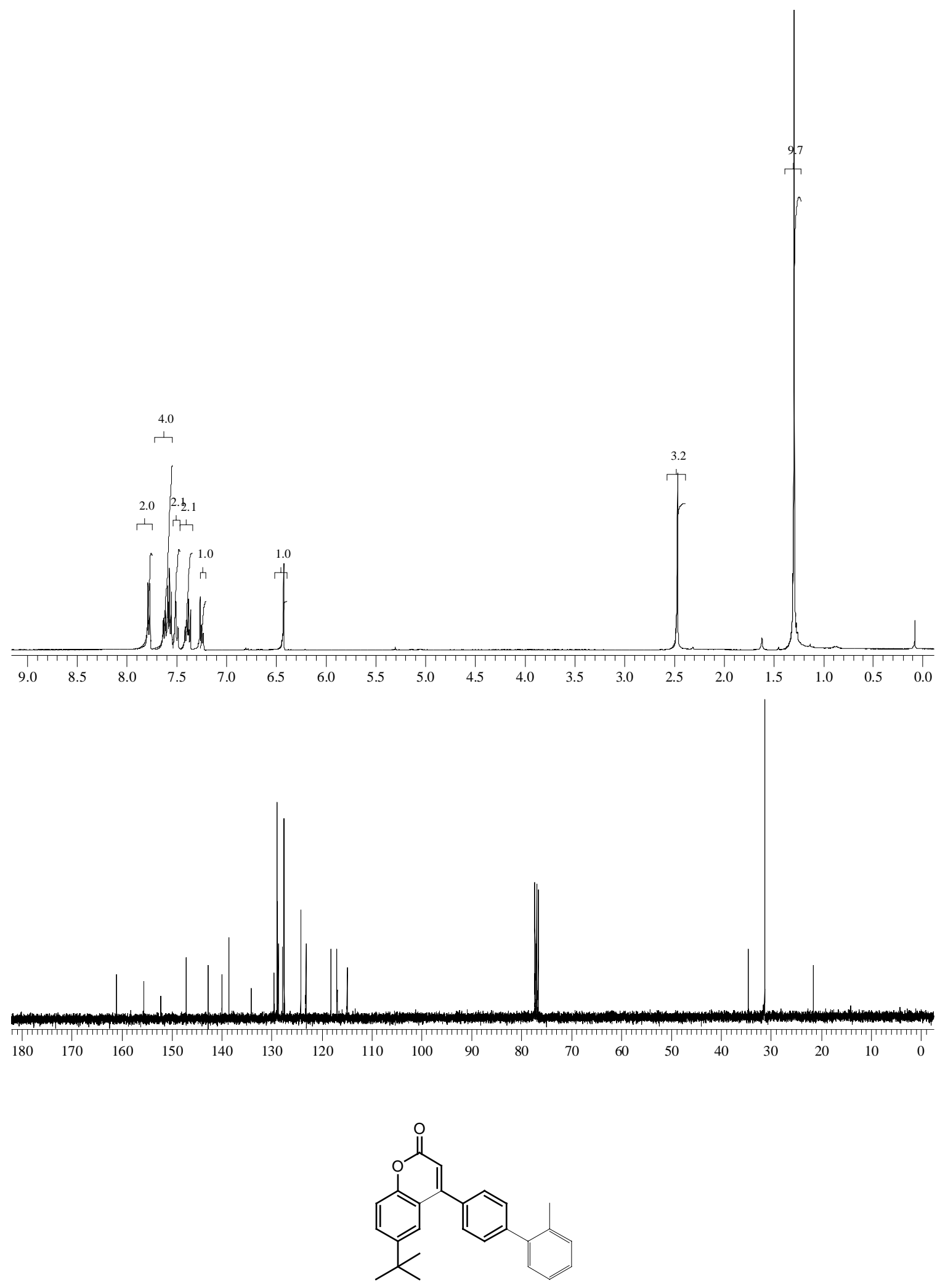

6-tert-Butyl-4-(4-o-tolylphenyl)-2H-chromen-2-one (3c). ${ }^{1} \mathrm{H} \mathrm{NMR}\left(\mathrm{CDCl}_{3}\right): \delta 7.62$ (dd, $1 \mathrm{H},{ }^{t} \mathrm{Bu} P h, J=8.8$ and $\left.2.3 \mathrm{~Hz}\right) ; 7.58\left(\mathrm{~d}, 1 \mathrm{H},{ }^{t} \mathrm{Bu} P h, J=2.3 \mathrm{~Hz}\right) ; 7.50-7.55(\mathrm{~m}, 4 \mathrm{H}$, 
$\left.P h P h \mathrm{CH}_{3}\right) ; 7.37\left(\mathrm{~d}, 1 \mathrm{H},{ }^{t} \mathrm{Bu} P h, J=8.8 \mathrm{~Hz}\right) ; 7.30-7.34\left(\mathrm{~m}, 4 \mathrm{H}, P h P h \mathrm{CH}_{3}\right) ; 6.44(\mathrm{~s}, 1 \mathrm{H}$, $\mathrm{CHCO}$ ); 2.36 (s, 3H, $\mathrm{PhCH}_{3}$ ); 1.29 (s, 9H, ${ }^{t} \mathrm{Bu}$ ). ${ }^{13} \mathrm{C}\left\{{ }^{1} \mathrm{H}\right\}$ NMR: $\delta 161.1$ (CO), Ph ring and $\mathrm{C}=\mathrm{C}(155.7,152.2,147.2,143.4,140.7,135.2,133.7,130.5,129.7,129.6,129.55$, 128.3, 127.7, 125.9, 123.1, 118.1, 116.9, 114.9), ${ }^{t} \mathrm{Bu}(34.6,31.3), 20.5\left(\mathrm{PhCH}_{3}\right) . v_{\max }$ $(\mathrm{NaCl}) / \mathrm{cm}^{-1}: 3058,1720,1616,1570,1485,1369,1186,1130$. HR-MS: $\mathrm{C}_{26} \mathrm{H}_{25} \mathrm{O}_{2}$ Calcd. $369.1855(\mathrm{M}+1)$; found: 369.1867 .

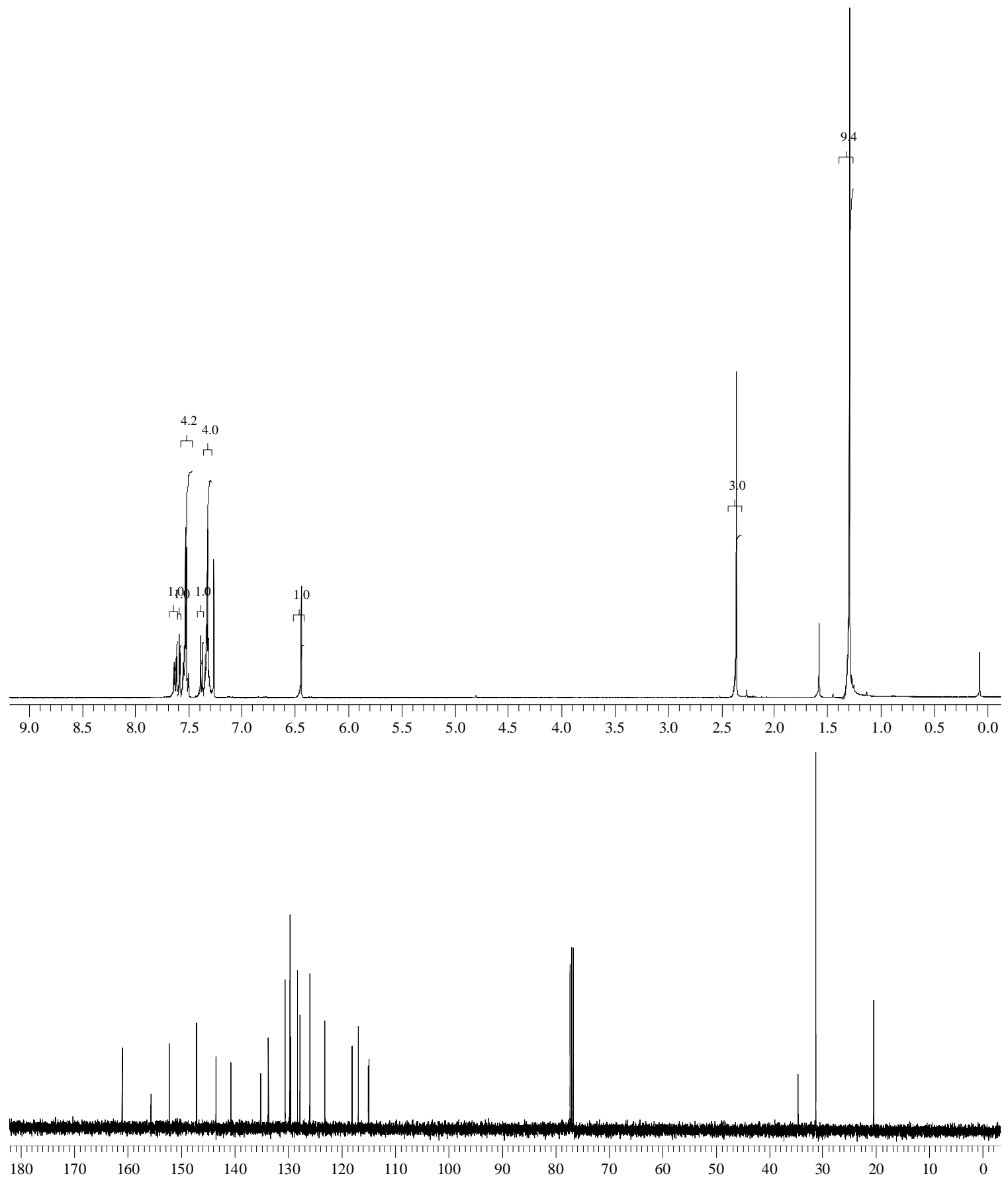




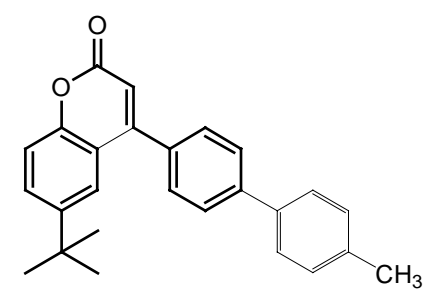

6-tert-Butyl-4-(4-p-tolylphenyl)-2H-chromen-2-one (3d). ${ }^{1} \mathrm{H} \mathrm{NMR}\left(\mathrm{CDCl}_{3}\right): \delta 7.77(\mathrm{~d}$, $\left.2 \mathrm{H}, P h \mathrm{PhCH}_{3}, J=8.2 \mathrm{~Hz}\right) ; 7.62\left(\mathrm{dd}, 1 \mathrm{H},{ }^{t} \mathrm{Bu} P h, J=8.8\right.$ and $\left.2.3 \mathrm{~Hz}\right) ; 7.60\left(\mathrm{~d}, 1 \mathrm{H},{ }^{t} \mathrm{Bu} P h\right.$, $J=2.3 \mathrm{~Hz}) ; 7.59$ (d, 2H, $\left.P h \mathrm{CH}_{3}, J=8.5 \mathrm{~Hz}\right) ; 7.56$ (d, 2H, $\left.P h \mathrm{PhCH}_{3}, J=8.2 \mathrm{~Hz}\right) ; 7.37$ (d, $1 \mathrm{H},{ }^{t} \mathrm{Bu} P h, J=8.8 \mathrm{~Hz}$ ); 7.31 (d, $2 \mathrm{H}, P h \mathrm{CH}_{3}, J=8.5 \mathrm{~Hz}$ ); 6.42 (s, 1H, CHCO); 2.43 (s, $\left.3 \mathrm{H}, \mathrm{PhCH}_{3}\right) ; 1.29\left(\mathrm{~s}, 9 \mathrm{H},{ }^{t} \mathrm{Bu}\right) .{ }^{13} \mathrm{C}\left\{{ }^{1} \mathrm{H}\right\} \mathrm{NMR}: \delta 161.1(\mathrm{CO}), \mathrm{Ph}$ ring and $\mathrm{C}=\mathrm{C}(155.6$, 152.2, 147.2, 142.5, 137.8, 137.0, 133.8, 129.7, 129.5, 128.9, 127.2, 126.9, 123.2, 118.1, 116.9, 114.8), ${ }^{t} \mathrm{Bu}(34.6,31.3), 21.1\left(\mathrm{PhCH}_{3}\right) \cdot v_{\max }(\mathrm{NaCl}) / \mathrm{cm}^{-1}: 3055,1720,1614,1570$, 1499, 1489, 1371, 1186, 1130. HR-MS: $\mathrm{C}_{26} \mathrm{H}_{25} \mathrm{O}_{2}$ Calcd. $369.1855(\mathrm{M}+1)$; found: 369.1851 .

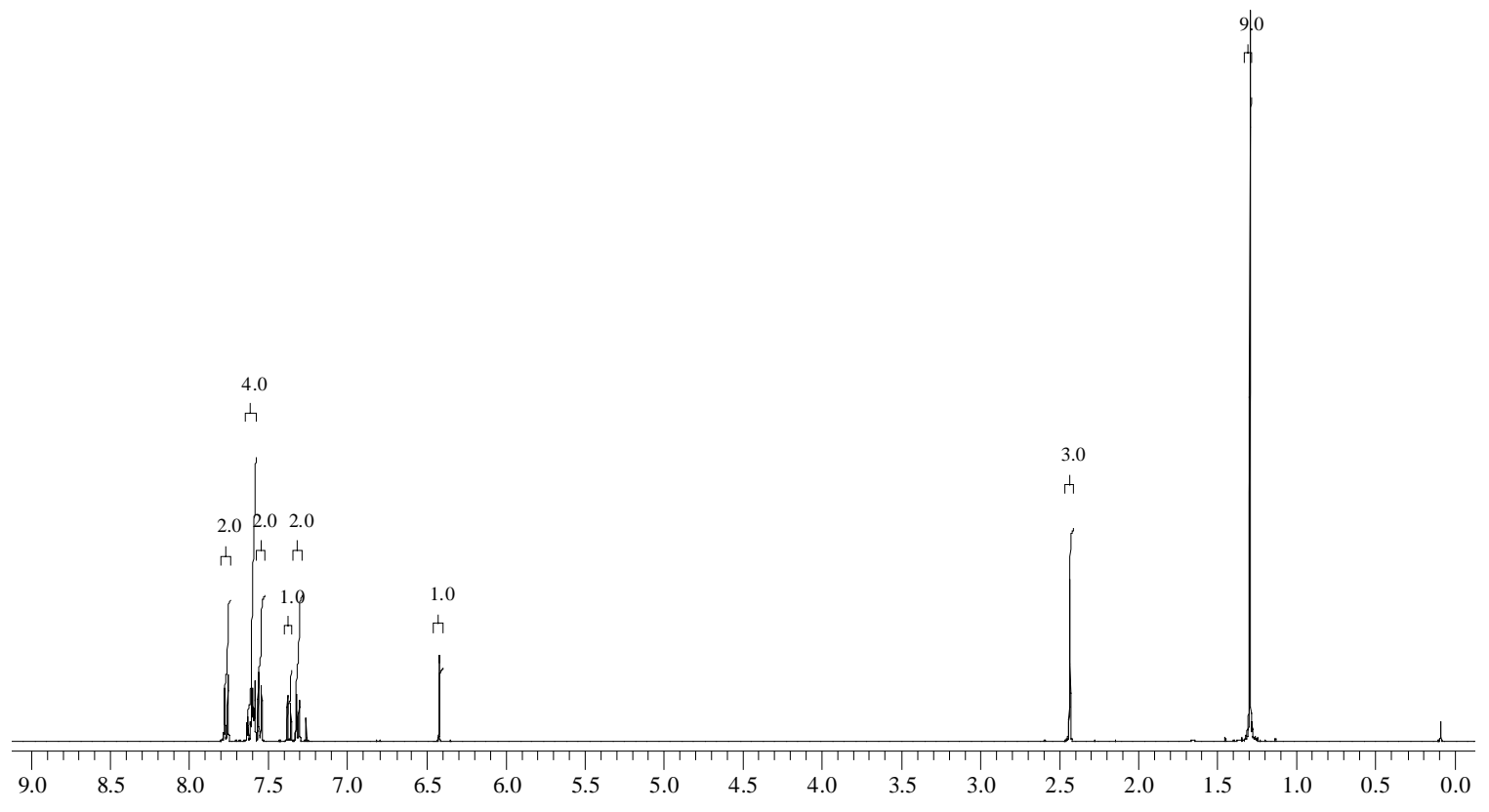




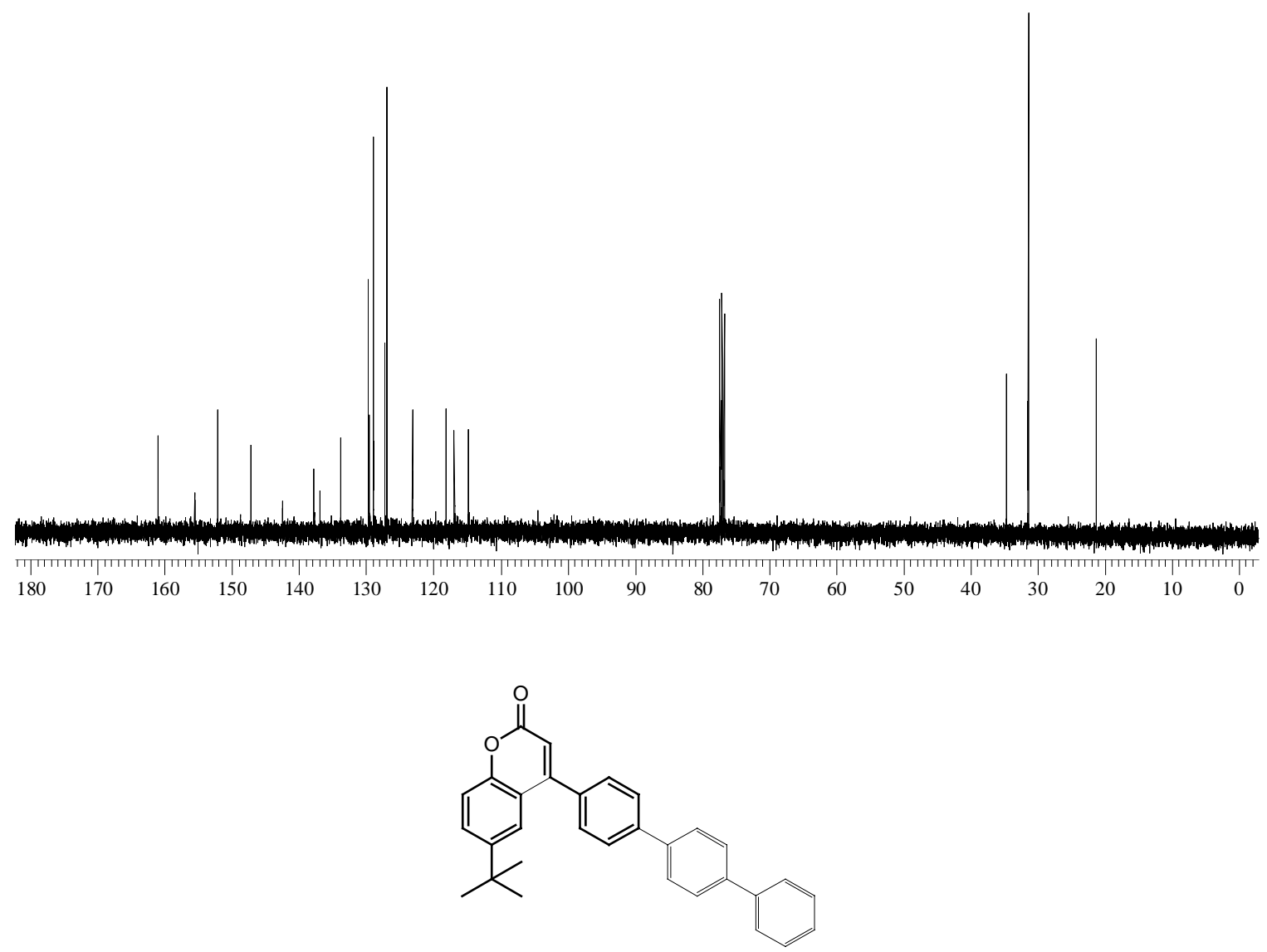

6-tert-Butyl-4-(4-terphenyl)-2H-chromen-2-one (3e). ${ }^{1} \mathrm{H}$ NMR $\left(\mathrm{CDCl}_{3}\right): \delta 7.83(\mathrm{~d}, 2 \mathrm{H}$, $\mathrm{PhPhPhC}, J=8.5 \mathrm{~Hz}$ ); 7.78 (d, 2H, PhPhC, $J=8.2 \mathrm{~Hz}$ ); 7.74 (d, 2H, $\mathrm{PhPhPhC}, J=8.5$ $\mathrm{Hz}) ; 7.67(\mathrm{~d}, 2 \mathrm{H}, P h \mathrm{PhPhC}, J=7.3 \mathrm{~Hz}) ; 7.62\left(\mathrm{dd}, 1 \mathrm{H},{ }^{t} \mathrm{Bu} P h, J=8.8\right.$ and $2.3 \mathrm{~Hz}$ ); 7.60 (s, $\left.1 \mathrm{H},{ }^{t} \mathrm{Bu} P h\right) ; 7.59$ (d, 2H, PhPhC, $\left.J=8.2 \mathrm{~Hz}\right) ; 7.49$ (t, 2H, $\left.P h \mathrm{PhPhC}, J=7.3 \mathrm{~Hz}\right) ; 7.39$ (t, $1 \mathrm{H}, P h \mathrm{PhPhC}, J=7.3 \mathrm{~Hz}$ ); $7.38\left(\mathrm{~d}, 1 \mathrm{H},{ }^{t} \mathrm{Bu} P h, J=8.8 \mathrm{~Hz}\right) ; 6.43$ (s, 1H, CHCO); 1.30 (s, $\left.9 \mathrm{H},{ }^{t} \mathrm{Bu}\right) .{ }^{13} \mathrm{C}\left\{{ }^{1} \mathrm{H}\right\}$ NMR: $\delta 161.1(\mathrm{CO}), \mathrm{Ph}$ ring and $\mathrm{C}=\mathrm{C}(155.5,152.2,147.2,142.0$, $140.8,140.4$, 138.7, 134.2, 129.6, 129.0, 128.8, 127.7, 127.52, 127.47, 127.3, 127.0, 123.1, 118.1, 116.9, 114.9), ${ }^{t} \mathrm{Bu}(34.6,31.3) . v_{\max }(\mathrm{NaCl}) / \mathrm{cm}^{-1}: 3055,1722,1614,1570$, 1485, 1371, 1184, 1130. HR-MS: $\mathrm{C}_{31} \mathrm{H}_{27} \mathrm{O}_{2}$ Calcd. 431.2011 (M+1); found: 431.2015. 


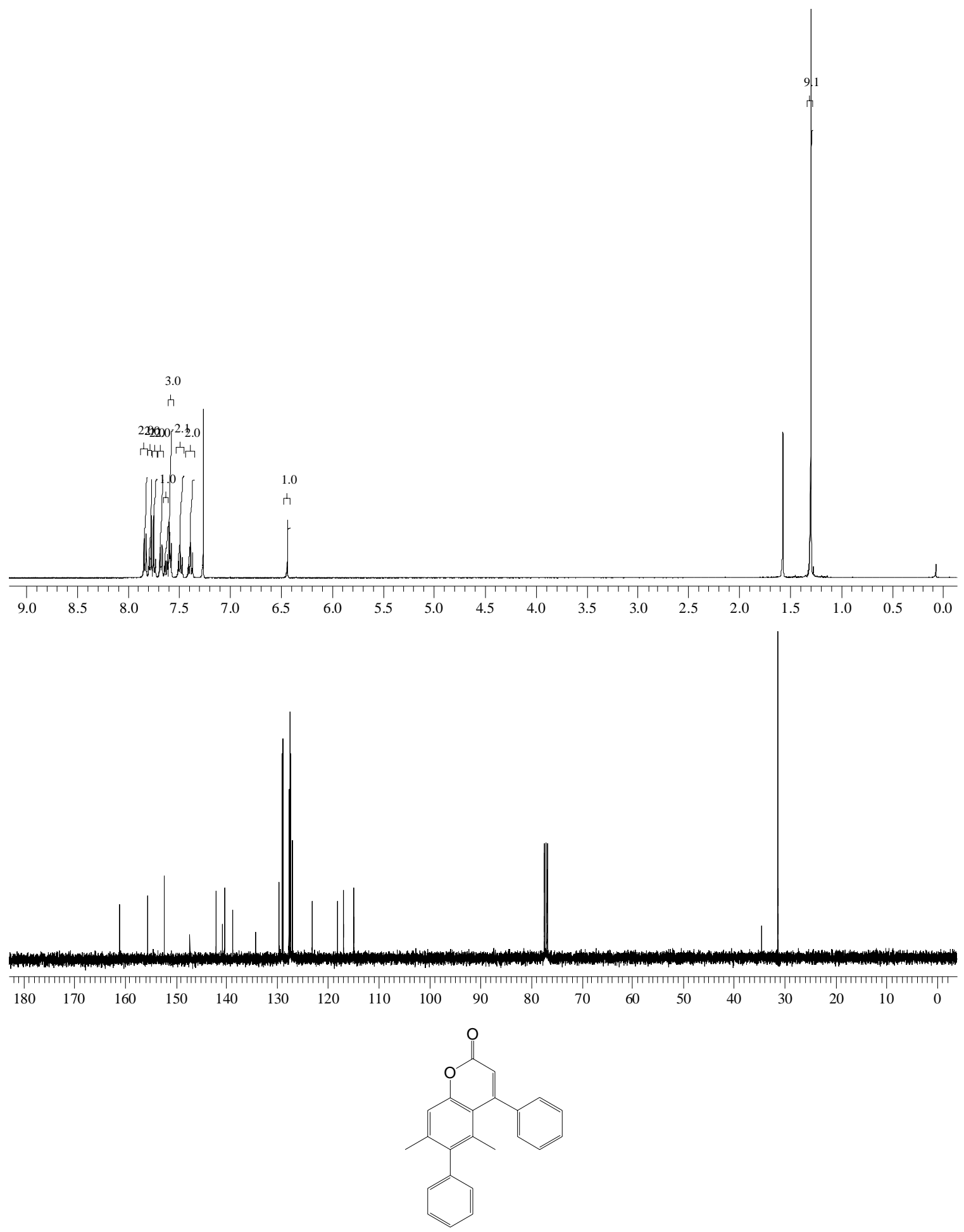

5,7-Dimethyl-4,6-diphenyl-2H-chromen-2-one (3f). ${ }^{1} \mathrm{H} \mathrm{NMR}\left(\mathrm{CDCl}_{3}\right): \delta$ 7.38-7.44 (m, $6 \mathrm{H}, P h \mathrm{C}=\mathrm{CH}, P h \mathrm{PhO}) ; 7.29-7.34(\mathrm{~m}, 3 \mathrm{H}, P h \mathrm{C}=\mathrm{CH}, P h \mathrm{PhO}) ; 7.21\left(\mathrm{~s}, 1 \mathrm{H}, P h \mathrm{CH}_{3}\right) ; 7.05$ (dd, 2H, PhPhO, $J=6.7$ and 1.5 Hz); 6.24 (s, 1H, CHCO); 2.07 (s, 3H, $\left.\mathrm{PhCH}_{3}\right) ; 1.50$ (s, $\left.3 \mathrm{H}, \mathrm{PhCH} H_{3}\right) .{ }^{13} \mathrm{C}\left\{{ }^{1} \mathrm{H}\right\} \mathrm{NMR}: \delta 160.5(\mathrm{CO}), \mathrm{Ph}$ ring and $\mathrm{C}=\mathrm{C}(157.0,154.0,141.3,140.4$, 
140.2, 140.0, 135.2, 129.1, 128.7, 128.6, 127.1, 127.0, 116.8, 116.1, 116.0), $\mathrm{PhCH}_{3}(21.8$, 21.7). $v_{\max }(\mathrm{NaCl}) / \mathrm{cm}^{-1}: 3055,1722,1602,1541,1357,1269,1211,1179,1120,1070$. HR-MS: $\mathrm{C}_{23} \mathrm{H}_{19} \mathrm{O}_{2}$ Calcd. $327.1385(\mathrm{M}+1)$; found: 327.1385.
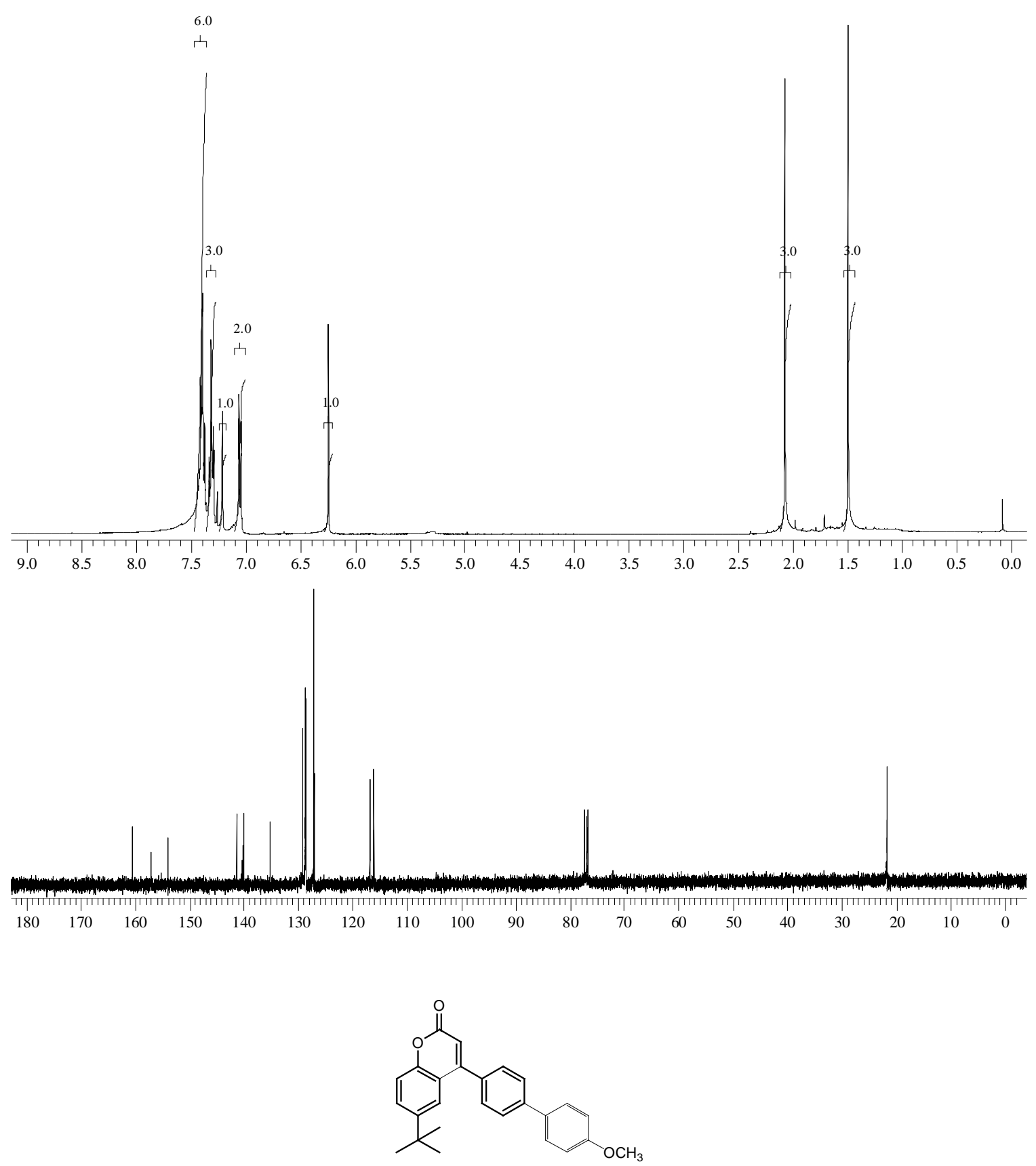

6-tert-Butyl-4-(4-p-methoxybiphenyl)-2H-chromen-2-one (3g). ${ }^{1} \mathrm{H}$ NMR $\left(\mathrm{CDCl}_{3}\right): \delta$ $7.74\left(\mathrm{~d}, 2 \mathrm{H}, P h \mathrm{PhOCH}_{3}, J=8.3 \mathrm{~Hz}\right) ; 7.63\left(\mathrm{~d}, 2 \mathrm{H}, P h \mathrm{OCH}_{3}, J=8.8 \mathrm{~Hz}\right) ; 7.60-7.64$ (m, $\left.2 \mathrm{H},{ }^{t} \mathrm{Bu} P h\right) ; 7.54\left(\mathrm{~d}, 2 \mathrm{H}, P h \mathrm{PhOCH}_{3}, J=8.3 \mathrm{~Hz}\right) ; 7.37$ (d, $\left.1 \mathrm{H},{ }^{t} \mathrm{Bu} P h, J=8.5 \mathrm{~Hz}\right) ; 7.03$ $\left(\mathrm{d}, 2 \mathrm{H}, \mathrm{PhOCH}_{3}, J=8.8 \mathrm{~Hz}\right) ; 6.41$ (s, $\left.1 \mathrm{H}, \mathrm{CHCO}\right) ; 3.88$ (s, 3H, $\left.\mathrm{OCH}_{3}\right) ; 1.29$ (s, 9H, $\left.{ }^{t} \mathrm{Bu}\right)$. 
${ }^{13} \mathrm{C}\left\{{ }^{1} \mathrm{H}\right\}$ NMR: $\delta 161.1(\mathrm{CO})$, Ph ring and $\mathrm{C}=\mathrm{C}(159.6,155.7,152.2,147.2,142.1,133.5$, 132.3, 129.5, 129.0, 128.2, 126.9, 123.2, 118.1, 116.9, 114.8, 114.4), $55.3\left(\mathrm{OCH}_{3}\right),{ }^{t} \mathrm{Bu}$ (34.6, 31.3). $v_{\max }(\mathrm{NaCl}) / \mathrm{cm}^{-1}:$ 3055, 1720, 1609, 1568, 1499, 1371, 1250, 1178, 1040. HR-MS: $\mathrm{C}_{26} \mathrm{H}_{25} \mathrm{O}_{3}$ Calcd. 385.1804 (M+1); found: 385.1800.

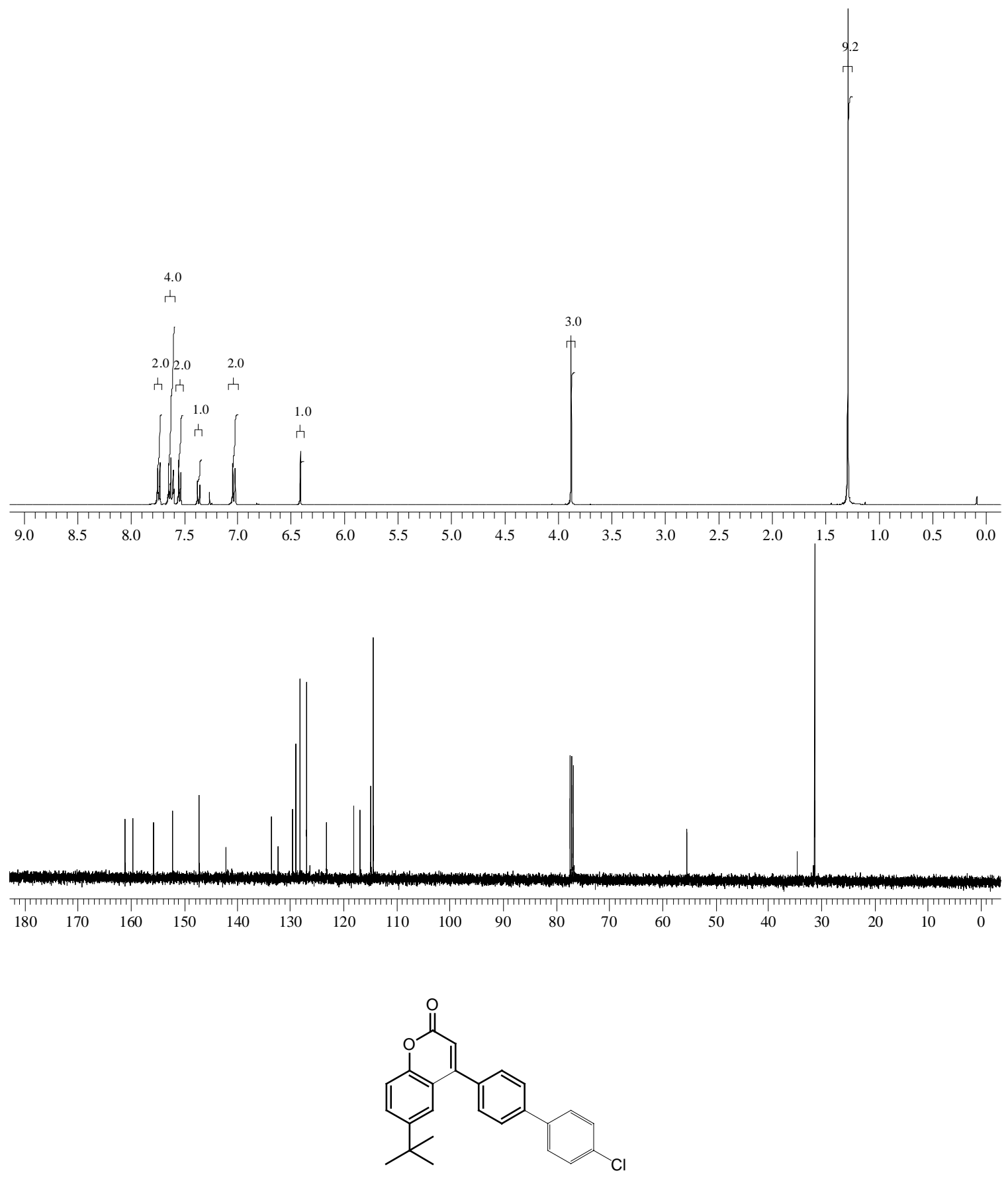

6-tert-Butyl-4-(4-p-chlorobiphenyl)-2H-chromen-2-one (3h). ${ }^{1} \mathrm{H}$ NMR $\left(\mathrm{CDCl}_{3}\right): \delta 7.74$ $(\mathrm{d}, 2 \mathrm{H}, \mathrm{Ph} P h \mathrm{C}, J=8.5 \mathrm{~Hz}) ; 7.62(\mathrm{~d}, 2 \mathrm{H}, \mathrm{PhCl}, J=8.5 \mathrm{~Hz}) ; 7.62\left(\mathrm{dd}, 1 \mathrm{H},{ }^{t} \mathrm{Bu} P h, J=8.8\right.$ 
and $2.3 \mathrm{~Hz}) ; 7.57(\mathrm{~d}, 2 \mathrm{H}, \mathrm{Ph} P h \mathrm{C}, J=8.5 \mathrm{~Hz}) ; 7.56\left(\mathrm{~s}, 1 \mathrm{H},{ }^{t} \mathrm{Bu} P h\right) ; 7.46(\mathrm{~d}, 2 \mathrm{H}, \mathrm{PhCl}, J=$ $8.5 \mathrm{~Hz}) ; 7.37\left(\mathrm{~d}, 1 \mathrm{H},{ }^{t} \mathrm{Bu} P h, J=8.8 \mathrm{~Hz}\right) ; 6.41(\mathrm{~s}, 1 \mathrm{H}, \mathrm{CHCO}) ; 1.29\left(\mathrm{~s}, 9 \mathrm{H},{ }^{t} \mathrm{Bu}\right) .{ }^{13} \mathrm{C}\left\{{ }^{1} \mathrm{H}\right\}$ NMR: $\delta 161.0(\mathrm{CO}), \mathrm{Ph}$ ring and $\mathrm{C}=\mathrm{C}(155.4,152.2,147.3,141.3,138.4,134.5,134.1$, $129.6,129.11,129.07,128.4,127.3,123.0,118.0,116.9,115.0),{ }^{t} \mathrm{Bu}(34.6,31.3) . v_{\max }$ $(\mathrm{NaCl}) / \mathrm{cm}^{-1}: 3055,1720,1616,1568,1558,1485,1371,1184,1130,1095$. HR-MS: $\mathrm{C}_{25} \mathrm{H}_{22} \mathrm{ClO}_{2}$ Calcd. 389.1308 (M+1); found: 389.1306.

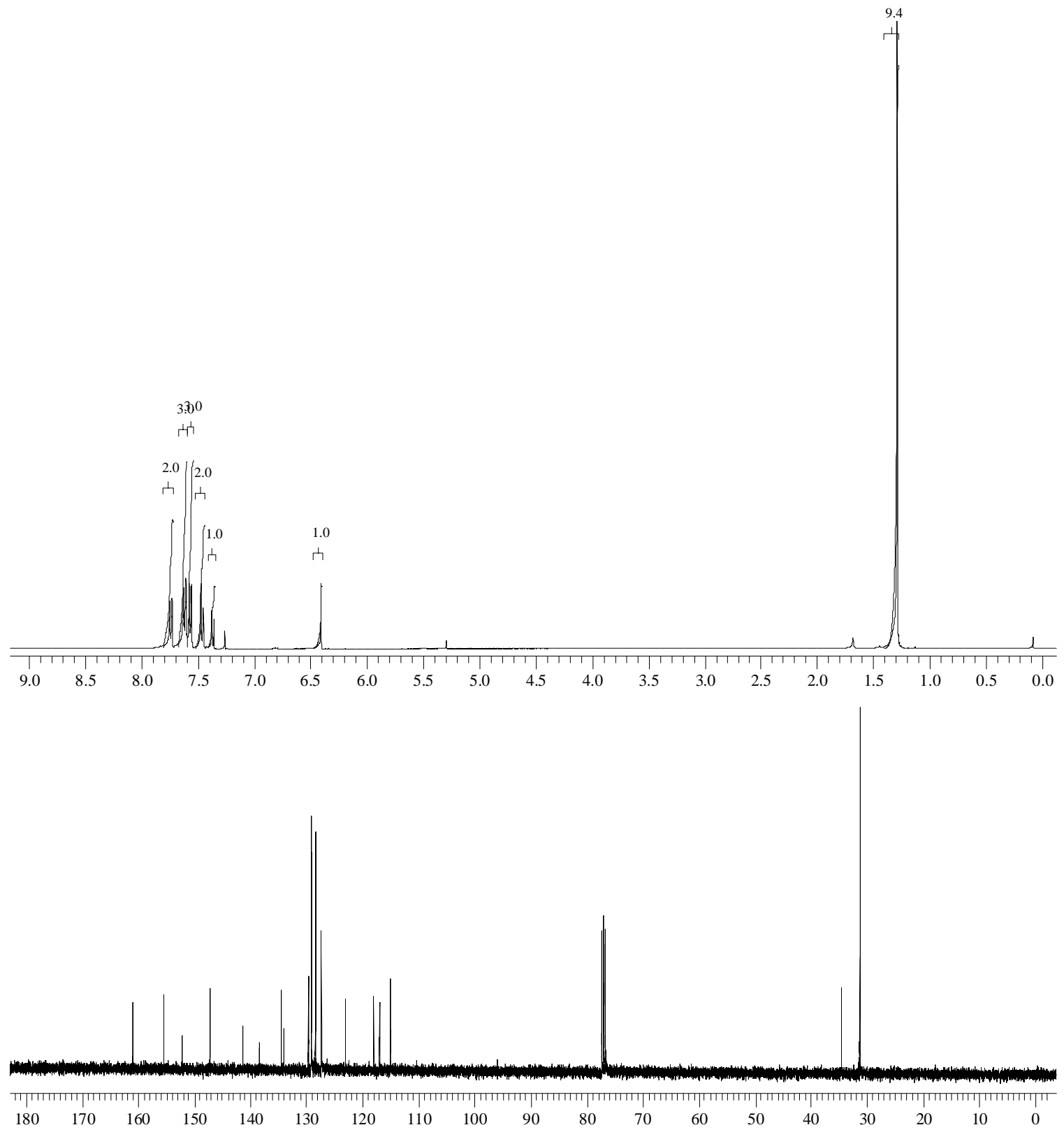




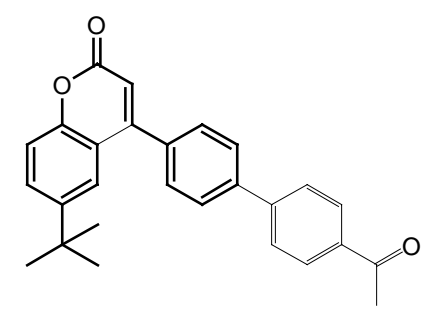

4-(4-p-Acetylbiphenyl)-6-tert-butyl-2H-chromen-2-one (3i). ${ }^{1} \mathrm{H}$ NMR $\left(\mathrm{CDCl}_{3}\right): \delta 8.09$ (d, $2 \mathrm{H}, P h \mathrm{COCH}_{3}, J=8.5 \mathrm{~Hz}$ ); $7.82(\mathrm{~d}, 2 \mathrm{H}, \mathrm{Ph} P h \mathrm{C}, J=8.5 \mathrm{~Hz}) ; .79$ (d, $2 \mathrm{H}, P h \mathrm{COCH}_{3}$, $J=8.5 \mathrm{~Hz}) ; 7.62\left(\mathrm{dd}, 1 \mathrm{H},{ }^{t} \mathrm{Bu} P h, J=8.8\right.$ and $\left.2.3 \mathrm{~Hz}\right) ; 7.60(\mathrm{~d}, 2 \mathrm{H}, \mathrm{Ph} P h \mathrm{C}, J=8.5 \mathrm{~Hz})$; $7.55\left(\mathrm{~d}, 1 \mathrm{H},{ }^{t} \mathrm{Bu} P h, J=2.3 \mathrm{~Hz}\right) ; 7.36\left(\mathrm{~d}, 1 \mathrm{H},{ }^{t} \mathrm{Bu} P h, J=8.8 \mathrm{~Hz}\right) ; 6.41$ (s, $\left.1 \mathrm{H}, \mathrm{CHCO}\right)$; 2.67 (s, 3H, $\left.\mathrm{COCH}_{3}\right), 1.29$ (s, 9H, $\left.{ }^{t} \mathrm{Bu}\right) .{ }^{13} \mathrm{C}\left\{{ }^{1} \mathrm{H}\right\}$ NMR: $\delta 197.6\left(\mathrm{COCH}_{3}\right), 160.9\left(\mathrm{CO}_{2}\right)$, $\mathrm{Ph}$ ring and $\mathrm{C}=\mathrm{C}(155.3,152.2,147.3,144.4,141.2,136.3,135.2,129.6,129.1,129.0$, 127.6, 127.3, 123.0, 118.0, 116.9, 115.1), ${ }^{t} \mathrm{Bu}(34.6,31.3), 26.7\left(\mathrm{COCH}_{3}\right) . v_{\max }$ $(\mathrm{NaCl}) / \mathrm{cm}^{-1}: 3058,1722,1684,1605,1570,1369,1360,1258,1184$. HR-MS: $\mathrm{C}_{27} \mathrm{H}_{25} \mathrm{O}_{3}$ Calcd. $397.1804(\mathrm{M}+1)$; found: 397.1799 .

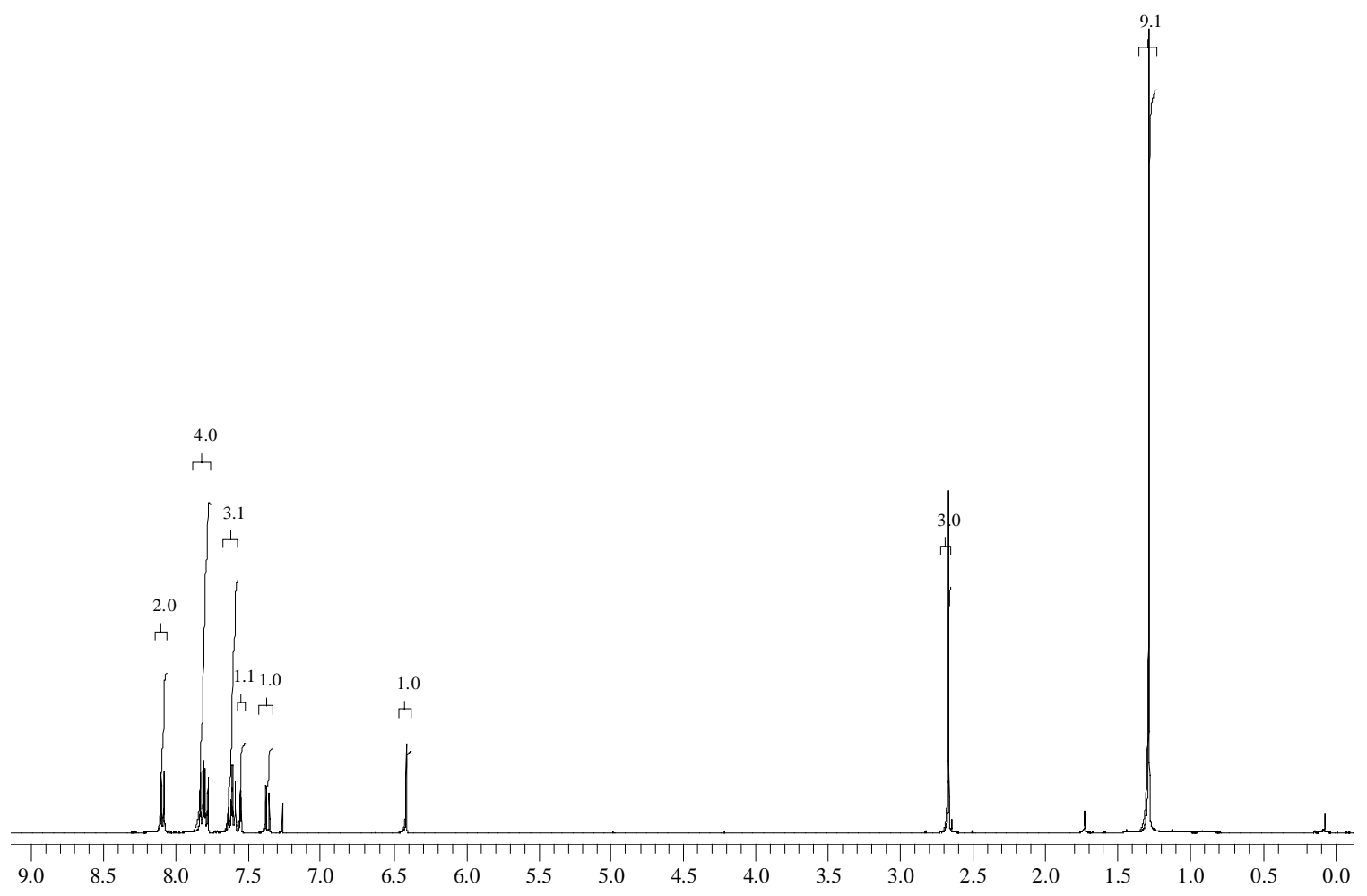



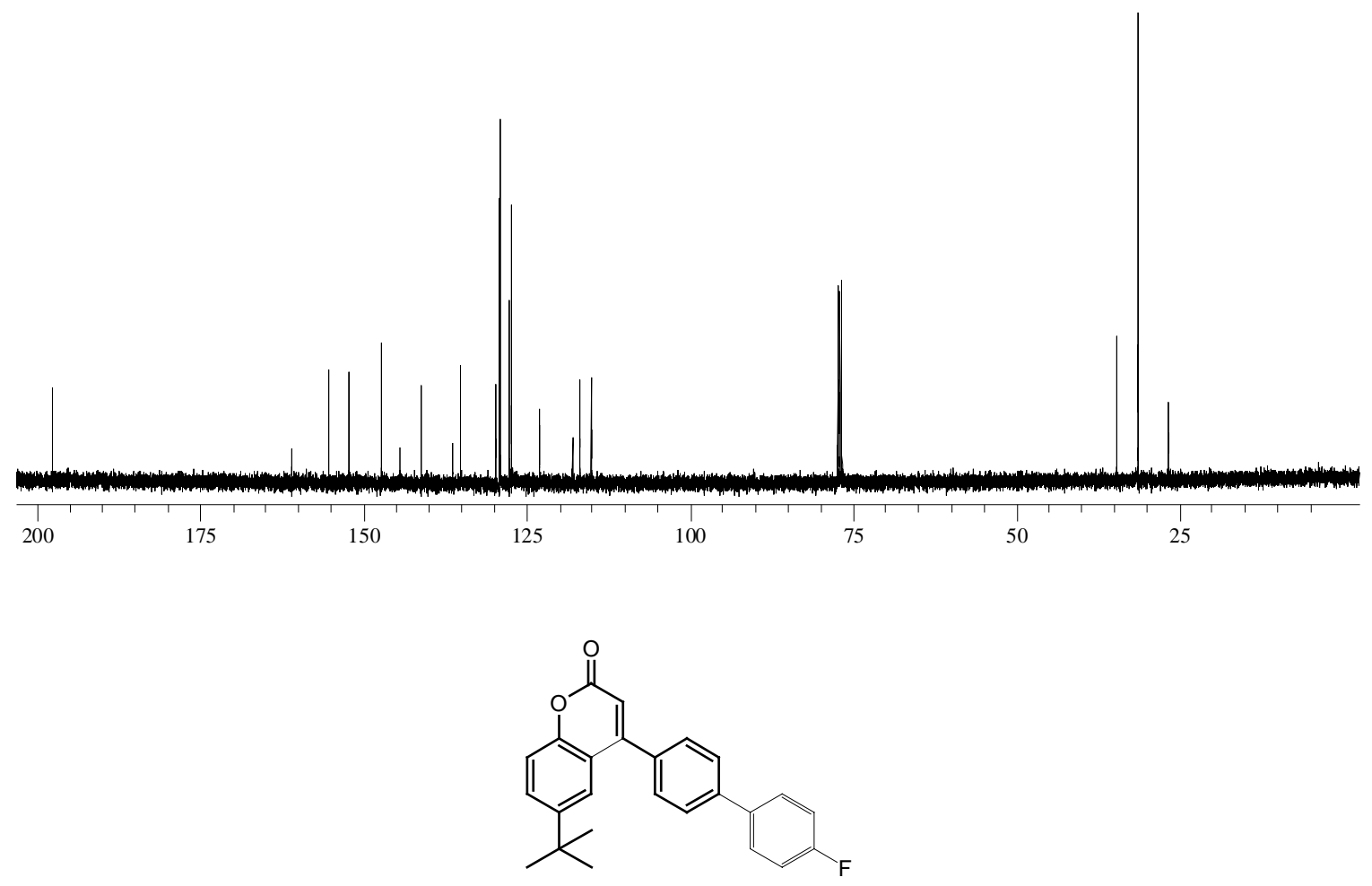

6-tert-Butyl-4-(4-p-fluorobiphenyl)-2H-chromen-2-one (3j). ${ }^{1} \mathrm{H}$ NMR $\left(\mathrm{CDCl}_{3}\right): \delta 7.73$ $(\mathrm{d}, 2 \mathrm{H}, \mathrm{Ph} P h \mathrm{C}, J=8.5 \mathrm{~Hz}) ; 7.65(\mathrm{dd}, 2 \mathrm{H}, \mathrm{PhF}, J=9.1$ and $5.3 \mathrm{~Hz}) ; 7.62\left(\mathrm{dd}, 1 \mathrm{H},{ }^{t} \mathrm{Bu} P h\right.$, $J=8.8$ and $2.3 \mathrm{~Hz}) ; 7.57\left(\mathrm{~d}, 1 \mathrm{H},{ }^{t} \mathrm{Bu} P h, J=2.3 \mathrm{~Hz}\right) ; 7.56(\mathrm{~d}, 2 \mathrm{H}, \mathrm{Ph} P h \mathrm{C}, J=8.5 \mathrm{~Hz})$; $7.37\left(\mathrm{~d}, 1 \mathrm{H},{ }^{t} \mathrm{Bu} P h, J=8.8 \mathrm{~Hz}\right) ; 7.19$ (t, $\left.2 \mathrm{H}, \mathrm{PhF}, J=8.8 \mathrm{~Hz}\right) ; 6.41$ (s, 1H, CHCO); 1.29 $\left(\mathrm{s}, 9 \mathrm{H},{ }^{t} \mathrm{Bu}\right) .{ }^{13} \mathrm{C}\left\{{ }^{1} \mathrm{H}\right\}$ NMR: $\delta 161.1(\mathrm{CO}), \mathrm{Ph}$ ring and $\mathrm{C}=\mathrm{C}(164.0,161.6,155.5,152.2$, 147.2, 141.6, 136.1, 134.2, 129.6, 129.0, 128.8, 127.3, 123.1, 118.1, 116.9, 116.0, 115.8, 115.0), ${ }^{t} \mathrm{Bu}(34.6,31.3) . v_{\max }(\mathrm{NaCl}) / \mathrm{cm}^{-1}: 3055,1720,1614,1605,1570,1499,1371$, 1184, 1159, 1130. HR-MS: $\mathrm{C}_{25} \mathrm{H}_{22} \mathrm{O}_{2}$ F Calcd. $373.1604(\mathrm{M}+1)$; found: 373.1595. 


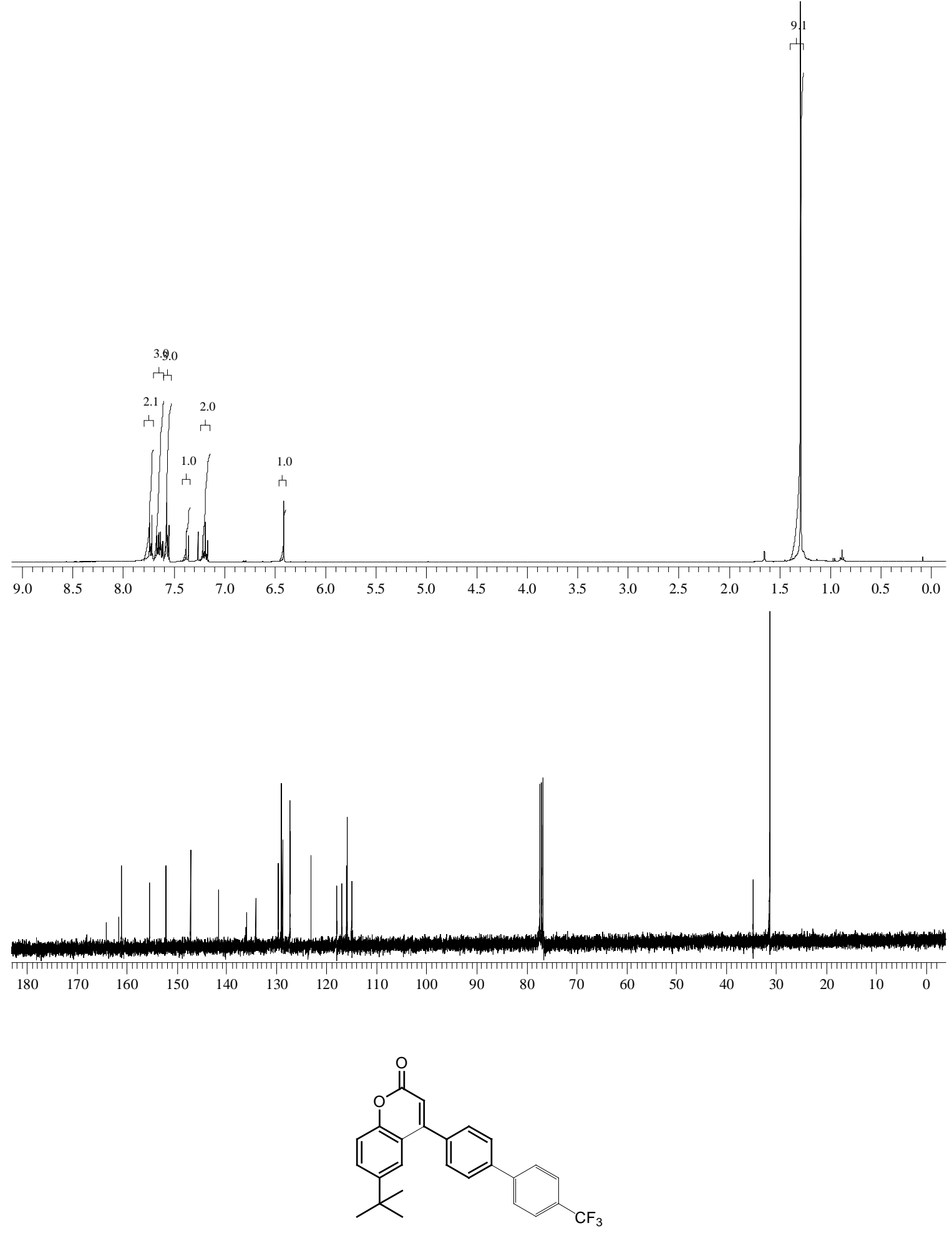

6-tert-Butyl-4-(4-p-trifluoromethylbiphenyl)-2H-chromen-2-one $\quad(3 k) . \quad{ }^{1} \mathrm{H} \quad \mathrm{NMR}$ $\left(\mathrm{CDCl}_{3}\right): \delta 7.80\left(\mathrm{~d}, 2 \mathrm{H}, \mathrm{PhCF}_{3}, J=8.5 \mathrm{~Hz}\right) ; 7.79(\mathrm{~d}, 2 \mathrm{H}, \mathrm{Ph} P h \mathrm{C}, J=8.5 \mathrm{~Hz}) ; 7.76(\mathrm{~d}, 2 \mathrm{H}$, $\left.\mathrm{PhCF}_{3}, J=8.5 \mathrm{~Hz}\right) ; 7.63\left(\mathrm{dd}, 1 \mathrm{H},{ }^{t} \mathrm{Bu} P h, J=8.8\right.$ and $\left.2.3 \mathrm{~Hz}\right) ; 7.60$ (d, 2H, $\mathrm{PhPhC}, J=$ 
$8.5 \mathrm{~Hz}) ; 7.55\left(\mathrm{~d}, 1 \mathrm{H},{ }^{t} \mathrm{Bu} P h, J=2.3 \mathrm{~Hz}\right) ; 7.37\left(\mathrm{~d}, 1 \mathrm{H},{ }^{t} \mathrm{Bu} P h, J=8.8 \mathrm{~Hz}\right) ; 6.42(\mathrm{~s}, 1 \mathrm{H}$, $\mathrm{CHCO}) ; 1.29$ (s, 9H, $\left.{ }^{t} \mathrm{Bu}\right) .{ }^{13} \mathrm{C}\left\{{ }^{1} \mathrm{H}\right\}$ NMR: $\delta 160.9(\mathrm{CO}), \mathrm{Ph}$ ring and $\mathrm{C}=\mathrm{C}(155.2,152.2$, 147.3 , 143.5, 141.0, 135.2, 129.7, 129.2, 127.7, 127.5, 125.96, 125.92, 125.88, 125.85, 123.0, 118.0, 117.0, 115.1), ${ }^{t} \mathrm{Bu}(34.6,31.3) . v_{\max }(\mathrm{NaCl}) / \mathrm{cm}^{-1}: 3059,1718,1703,1616$, 1464, 1396, 1326, 1128, 1070, 1020. HR-MS: $\mathrm{C}_{26} \mathrm{H}_{22} \mathrm{O}_{2} \mathrm{~F}_{3}$ Calcd. 423.1572 (M+1); found: 423.1568 .

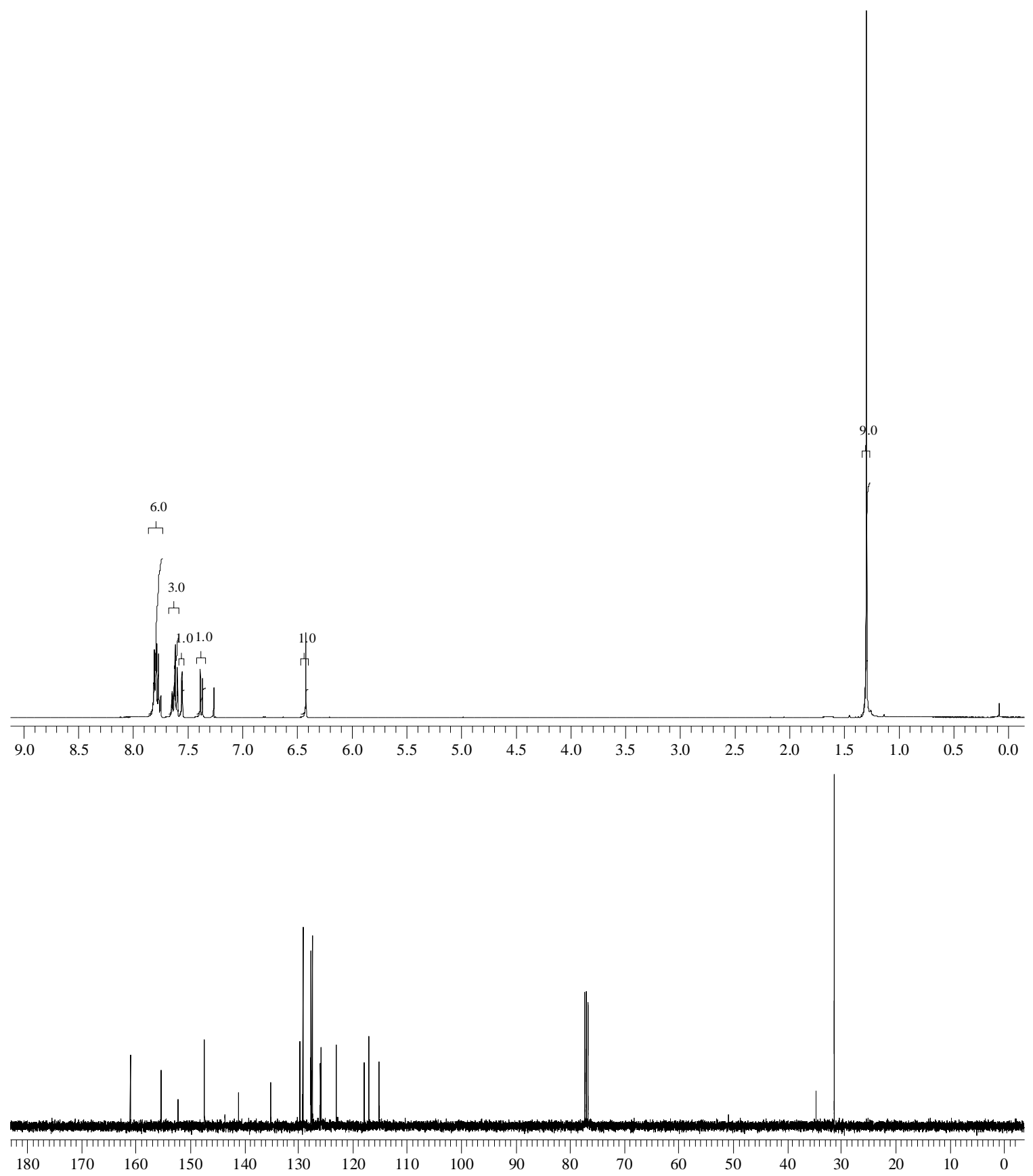




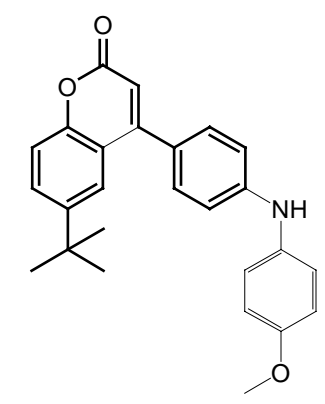

6-tert-Butyl-4-(4-p-methoxyanilinophenyl)-2H-chromen-2-one (31). ${ }^{1} \mathrm{H} \mathrm{NMR}\left(\mathrm{CDCl}_{3}\right)$ : $\delta 7.67\left(\mathrm{~d}, 1 \mathrm{H},{ }^{t} \mathrm{Bu} P h, J=2.3 \mathrm{~Hz}\right) ; 7.58\left(\mathrm{dd}, 1 \mathrm{H},{ }^{t} \mathrm{Bu} P h, J=8.5\right.$ and $\left.2.3 \mathrm{~Hz}\right) ; 7.34(\mathrm{~d}, 2 \mathrm{H}$, $\mathrm{NH} P h \mathrm{C}, J=8.5 \mathrm{~Hz}) ; 7.32\left(\mathrm{~d}, 1 \mathrm{H},{ }^{t} \mathrm{Bu} P h, J=8.5 \mathrm{~Hz}\right) ; 7.18\left(\mathrm{~d}, 2 \mathrm{H}, P h \mathrm{OCH}_{3}, J=8.8 \mathrm{~Hz}\right)$; $6.99(\mathrm{~d}, 2 \mathrm{H}, \mathrm{NHPhC}, J=8.5 \mathrm{~Hz}) ; 6.92\left(\mathrm{~d}, 2 \mathrm{H}, P h \mathrm{OCH}_{3}, J=8.8 \mathrm{~Hz}\right) ; 6.32(\mathrm{~s}, 1 \mathrm{H}$, $\mathrm{CHCO}$ ); 5.89 (s, br, 1H, NH), $3.82\left(\mathrm{~s}, 3 \mathrm{H}, \mathrm{OCH}_{3}\right), 1.29$ (s, 9H, $\left.{ }^{t} \mathrm{Bu}\right) .{ }^{13} \mathrm{C}\left\{{ }^{1} \mathrm{H}\right\}$ NMR: $\delta$ $161.5(\mathrm{CO}), \mathrm{Ph}$ ring and $\mathrm{C}=\mathrm{C}(156.1,155.8,152.2,147.2,146.9,134.0,129.9,129.2$, 125.4, 123.8, 123.3, 118.3, 116.8, 114.7, 114.3, 113.5), $55.5\left(\mathrm{OCH}_{3}\right),{ }^{t} \mathrm{Bu}(34.6,31.3)$. $v_{\max }(\mathrm{NaCl}) / \mathrm{cm}^{-1}: 3424,3055,1717,1705,1606,1510,1369,1186,1036$. HR-MS: $\mathrm{C}_{26} \mathrm{H}_{26} \mathrm{NO}_{3}$ Calcd. 400.1913 (M+1); found: 400.1929.

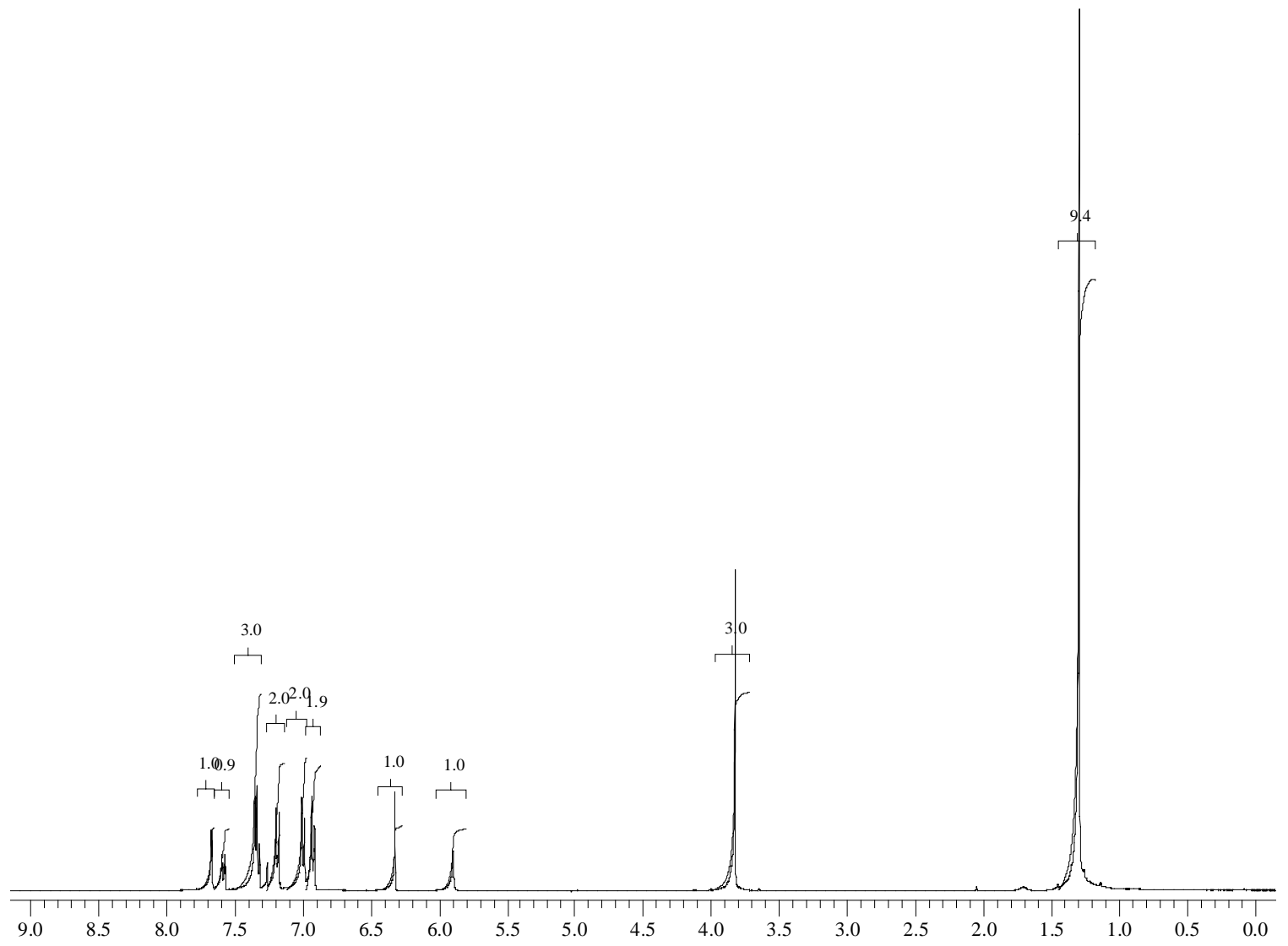



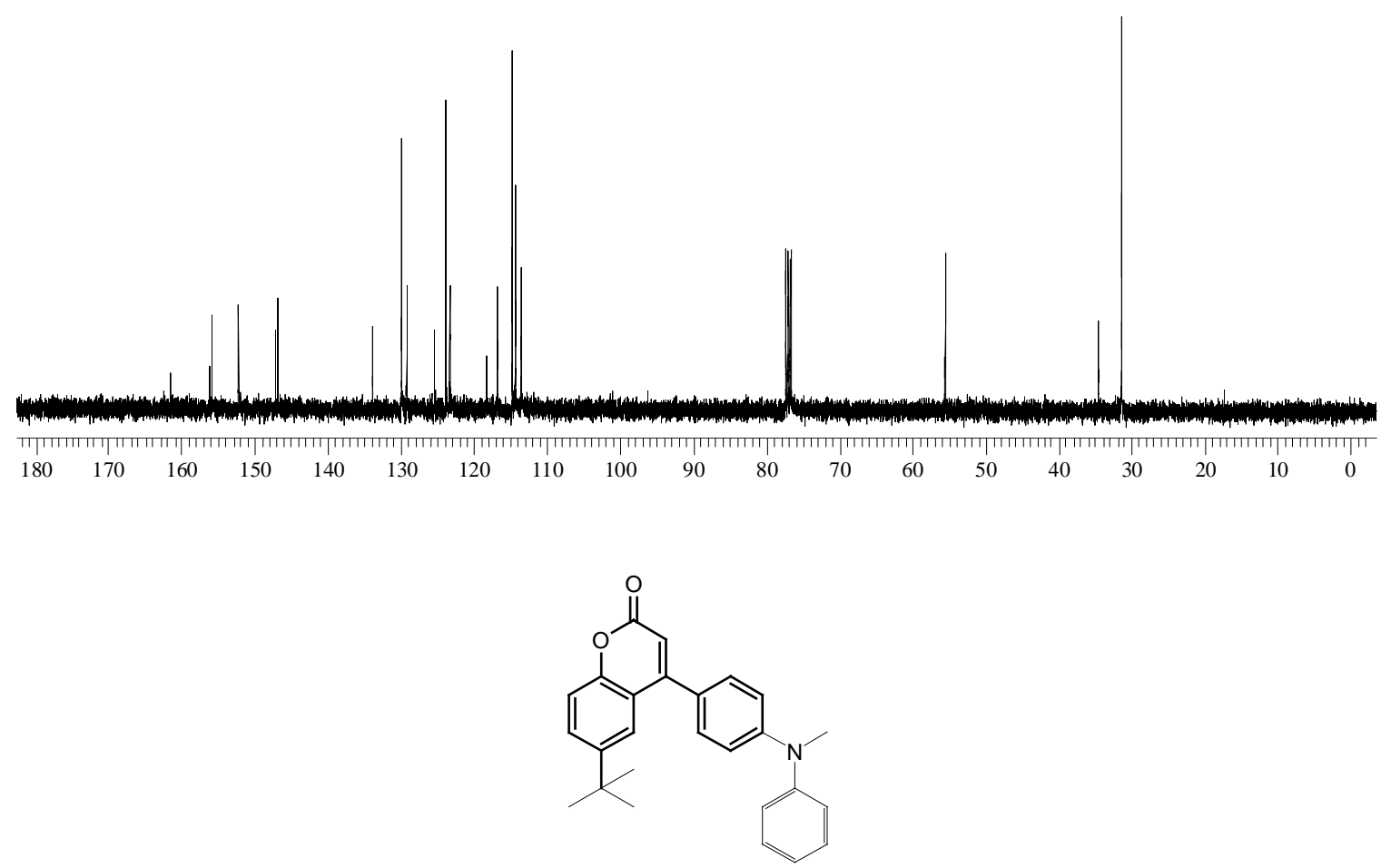

6-tert-Butyl-4-(4- $\mathrm{N}$-methyanilinophenyl)-2H-chromen-2-one (3m). ${ }^{1} \mathrm{H}$ NMR $\left(\mathrm{CDCl}_{3}\right)$ : $\delta 7.68\left(\mathrm{~d}, 1 \mathrm{H},{ }^{t} \mathrm{Bu} P h, J=2.3 \mathrm{~Hz}\right) ; 7.58\left(\mathrm{dd}, 1 \mathrm{H},{ }^{t} \mathrm{Bu} P h, J=8.8\right.$ and $\left.2.3 \mathrm{~Hz}\right) ; 7.41(\mathrm{t}, 2 \mathrm{H}$, $P h \mathrm{NPhC}=\mathrm{CH}, J=7.6 \mathrm{~Hz}) ; 7.36(\mathrm{~d}, 2 \mathrm{H}, P h \mathrm{C}=\mathrm{CH}, J=8.8 \mathrm{~Hz}) ; 7.26(\mathrm{dt}, 2 \mathrm{H}$, $P h \mathrm{NPhC}=\mathrm{CH}, J=7.3$ and $2.6 \mathrm{~Hz}) ; 7.34\left(\mathrm{~d}, 1 \mathrm{H},{ }^{t} \mathrm{Bu} P h, J=8.8 \mathrm{~Hz}\right) ; 7.19(\mathrm{t}, 1 \mathrm{H}$, $P h \mathrm{NPhC}=\mathrm{CH}, J=7.3 \mathrm{~Hz}) ; 6.98(\mathrm{~d}, 2 \mathrm{H}, P h \mathrm{C}=\mathrm{CH}, J=8.8 \mathrm{~Hz}) ; 6.33(\mathrm{~s}, 1 \mathrm{H}, \mathrm{CHCO}) ; 3.42$ $\left(\mathrm{s}, 3 \mathrm{H}, \mathrm{NCH}_{3}\right), 3.82\left(\mathrm{~s}, 3 \mathrm{H}, \mathrm{OCH}_{3}\right), 1.30\left(\mathrm{~s}, 9 \mathrm{H},{ }^{t} \mathrm{Bu}\right) .{ }^{13} \mathrm{C}\left\{{ }^{1} \mathrm{H}\right\} \mathrm{NMR}: \delta 161.5(\mathrm{CO}), \mathrm{Ph}$ ring and $\mathrm{C}=\mathrm{C}(155.8,152.3,150.2,147.8,146.9,129.7,129.6,129.2,125.0,124.9,124.6$, 123.3, 118.3, 116.8, 115.6, 113.5), $40.2\left(\mathrm{NCH}_{3}\right),{ }^{t} \mathrm{Bu}(34.6,31.3) . v_{\max }(\mathrm{NaCl}) / \mathrm{cm}^{-1}: 3058$, 1717, 1705, 1610, 1591, 1614, 1495, 1369, 1354, 1193, 1130. HR-MS: $\mathrm{C}_{26} \mathrm{H}_{26} \mathrm{NO}_{2}$ Calcd. $384.1964(\mathrm{M}+1)$; found: 384.1956. 

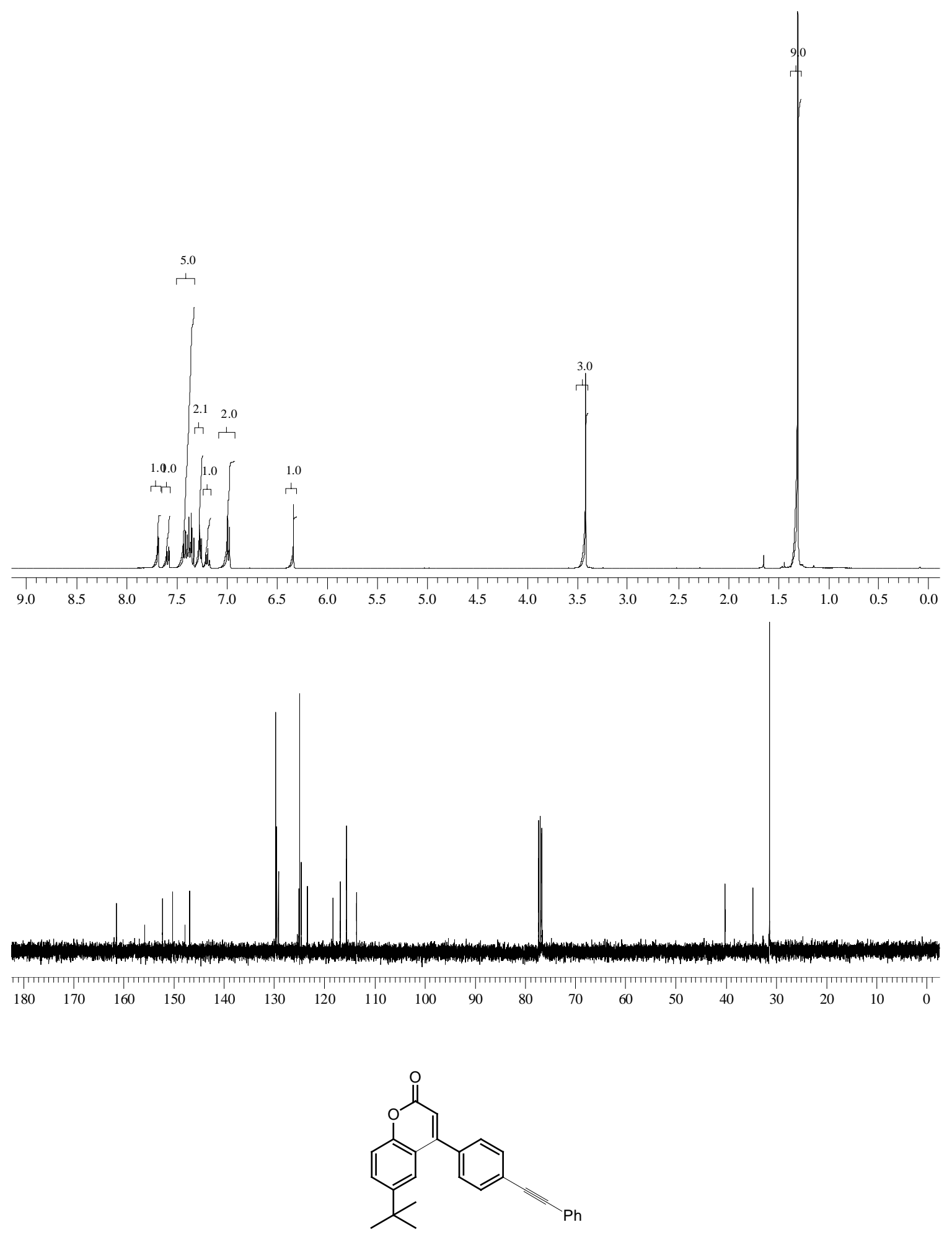

6-tert-Butyl-4-(4-p-phenylacetylenylphenyl)-2H-chromen-2-one $\quad$ (3n). ${ }^{1} \mathrm{H} \quad \mathrm{NMR}$ $\left(\mathrm{CDCl}_{3}\right): \delta 7.71(\mathrm{~d}, 2 \mathrm{H}, P h \mathrm{C}=\mathrm{CH}, J=8.2 \mathrm{~Hz}) ; 7.62\left(\mathrm{dd}, 1 \mathrm{H},{ }^{t} \mathrm{Bu} P h, J=8.8\right.$ and $\left.2.3 \mathrm{~Hz}\right)$; $7.58\left(\mathrm{dd}, 2 \mathrm{H}, \mathrm{PhC} \equiv \mathrm{C}, J=7.6\right.$ and 2.0Hz); $7.49\left(\mathrm{~d}, 1 \mathrm{H},{ }^{t} \mathrm{Bu} P h, J=2.3 \mathrm{~Hz}\right) ; .48(\mathrm{~d}, 2 \mathrm{H}$, 
$P h \mathrm{C}=\mathrm{CH}, J=8.2 \mathrm{~Hz}) ; 7.37-7.40(\mathrm{~m}, 3 \mathrm{H}, \mathrm{PhC} \equiv \mathrm{C}) ; 7.36\left(\mathrm{~d}, 1 \mathrm{H},{ }^{t} \mathrm{Bu} P h, J=8.8 \mathrm{~Hz}\right) ; 6.39$ (s, $1 \mathrm{H}, \mathrm{CHCO}) ; 1.28\left(\mathrm{~s}, 9 \mathrm{H},{ }^{t} \mathrm{Bu}\right) .{ }^{13} \mathrm{C}\left\{{ }^{1} \mathrm{H}\right\}$ NMR: $\delta 160.9(\mathrm{CO})$, $\mathrm{Ph}$ ring and $\mathrm{C}=\mathrm{C}(155.2$, 152.2, 147.3, 135.0, 132.0, 131.7, 129.7, 128.7, 128.5, 128.4, 124.9, 122.9, 122.7, 117.9, 116.9, 115.0), $\mathrm{C} \equiv \mathrm{C}(91.3,88.4),{ }^{t} \mathrm{Bu}(34.6,31.2) . v_{\max }(\mathrm{NaCl}) / \mathrm{cm}^{-1}: 3055,1720,1614$, 1570, 1510, 1371, 1186, 1018. HR-MS: $\mathrm{C}_{27} \mathrm{H}_{23} \mathrm{O}_{2}$ Calcd. $379.1698(\mathrm{M}+1)$; found: 379.1701 .

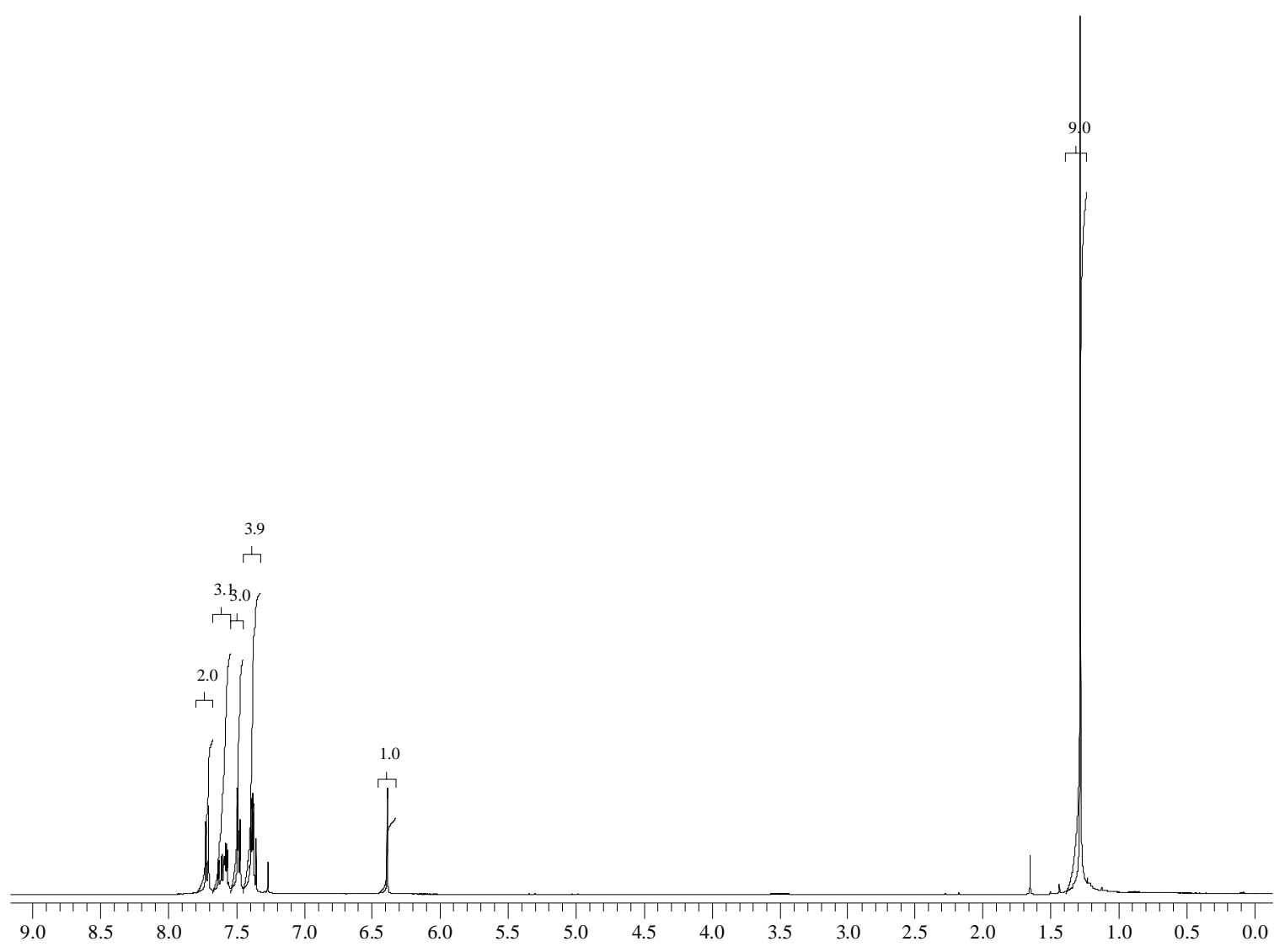




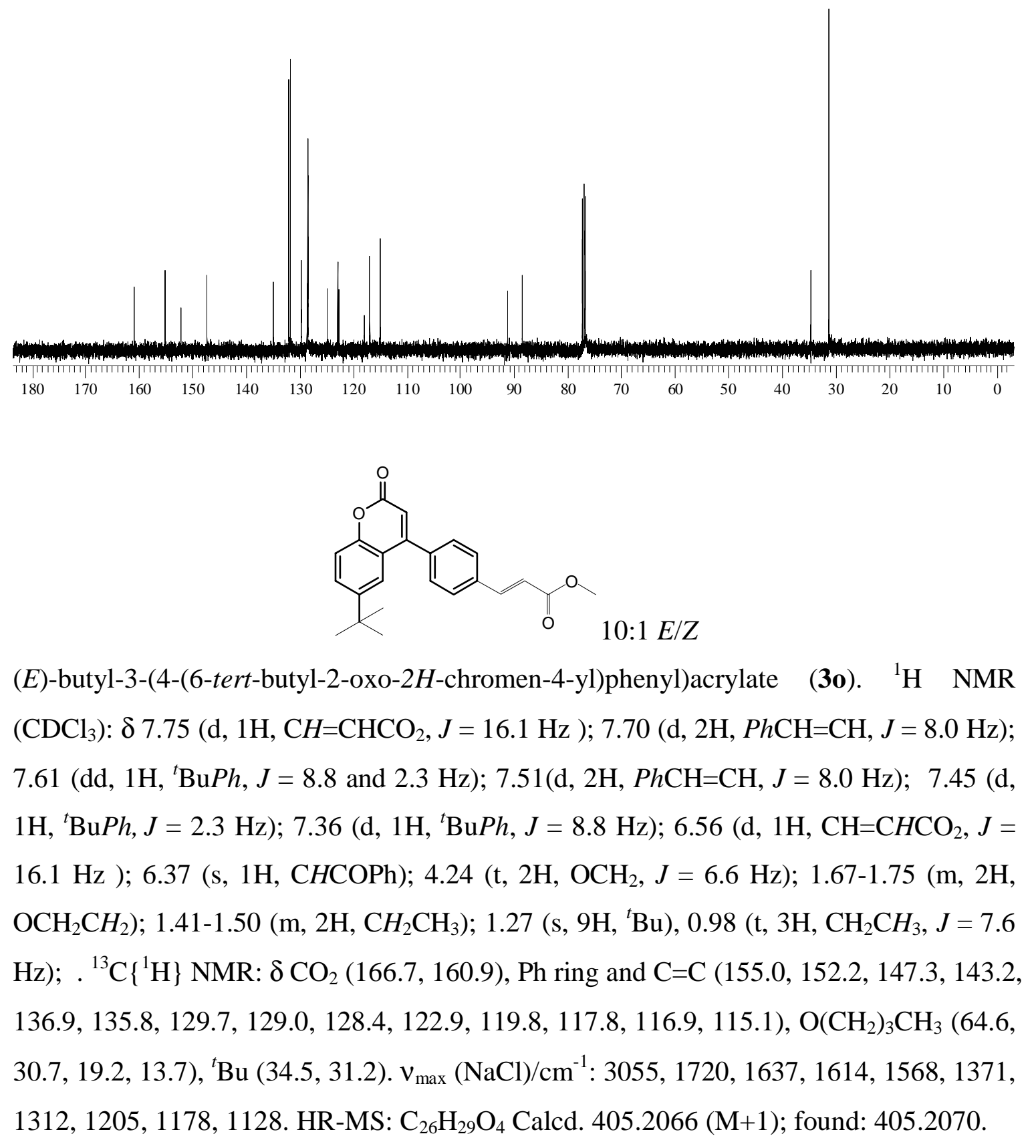




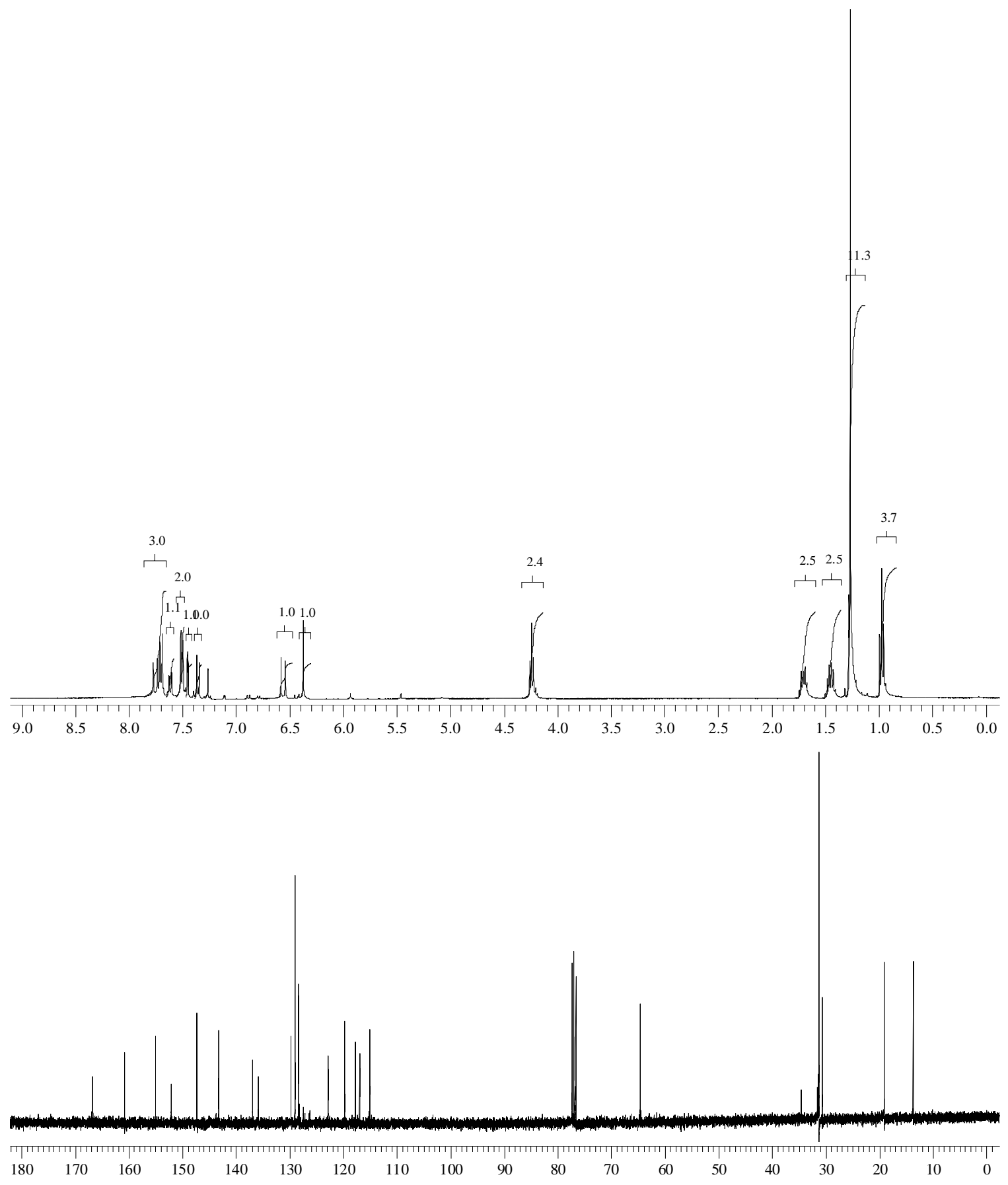

S-31 


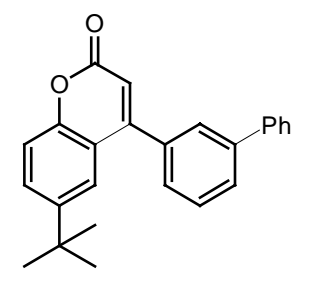

4-(3-Biphenyl)-6-tert-butyl-2H-chromen-2-one (3p). ${ }^{1} \mathrm{H}$ NMR $\left(\mathrm{CDCl}_{3}\right): \delta 7.77(\mathrm{~d}, 1 \mathrm{H}$, $\mathrm{Ph} P h \mathrm{C}, J=7.9 \mathrm{~Hz}$ ); 7.70 (s, 1H, $\mathrm{Ph} P h \mathrm{C}$ ); 7.60-7.65 (m, 4H, ${ }^{t} \mathrm{BuPh}, 1 \mathrm{H} ; \mathrm{Ph} P h \mathrm{C}, 1 \mathrm{H}$; $P h \mathrm{PhC}, 2 \mathrm{H}) ; 7.57\left(\mathrm{~d}, 1 \mathrm{H},{ }^{t} \mathrm{Bu} P h, J=2.0 \mathrm{~Hz}\right) ; 7.48(\mathrm{t}, 2 \mathrm{H}, P h \mathrm{PhC}, J=7.6 \mathrm{~Hz}) ; 7.46(\mathrm{t}$, $1 \mathrm{H}, \mathrm{Ph} P h \mathrm{C}, J=7.6 \mathrm{~Hz}$ ); 7.40 (t, $1 \mathrm{H}, P h \mathrm{PhC}, J=7.6 \mathrm{~Hz}$ ); 7.37 (d, 1H, ${ }^{t} \mathrm{Bu} P h, J=8.8 \mathrm{~Hz}$ ); 6.43 (s, $1 \mathrm{H}, \mathrm{CHCO}) ; 1.28\left(\mathrm{~s}, 9 \mathrm{H},{ }^{t} \mathrm{Bu}\right) \cdot{ }^{13} \mathrm{C}\left\{{ }^{1} \mathrm{H}\right\}$ NMR: $\delta 161.0(\mathrm{CO}), \mathrm{Ph}$ ring and $\mathrm{C}=\mathrm{C}$ $(155.8,152.2,147.2,141.9,140.0,135.8,129.6,129.2,128.9,128.4,127.8,127.2$, 127.14, 127.07, 123.1, 118.1, 116.9, 115.1), ${ }^{t} \mathrm{Bu}(34.6,31.3) . v_{\max }(\mathrm{NaCl}) / \mathrm{cm}^{-1}: 3058$, 1722, 1616, 1566, 1479, 1369, 1184, 1128. HR-MS: $\mathrm{C}_{25} \mathrm{H}_{23} \mathrm{O}_{2}$ Calcd. $355.1698(\mathrm{M}+1)$; found: 355.1695 .

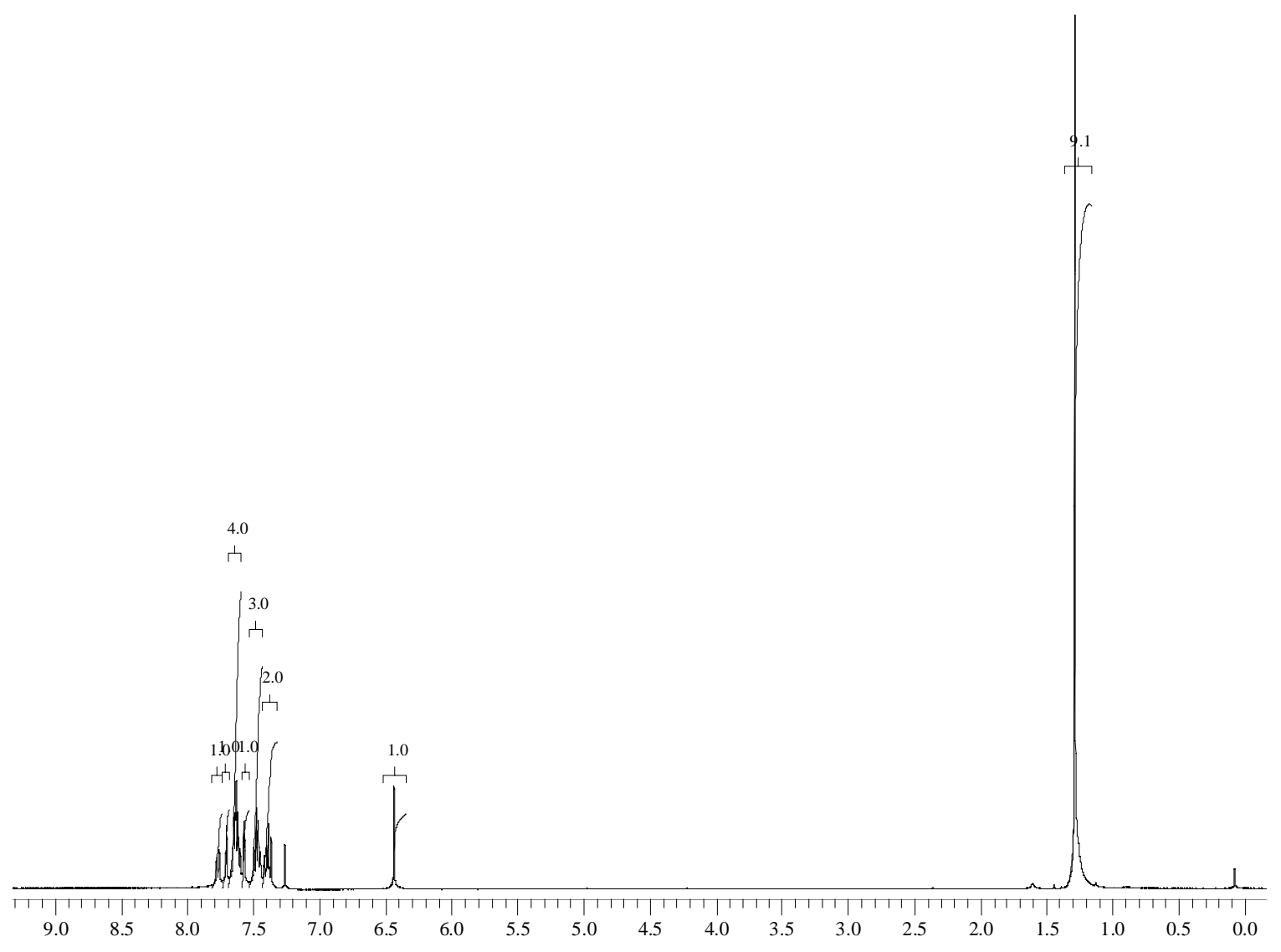



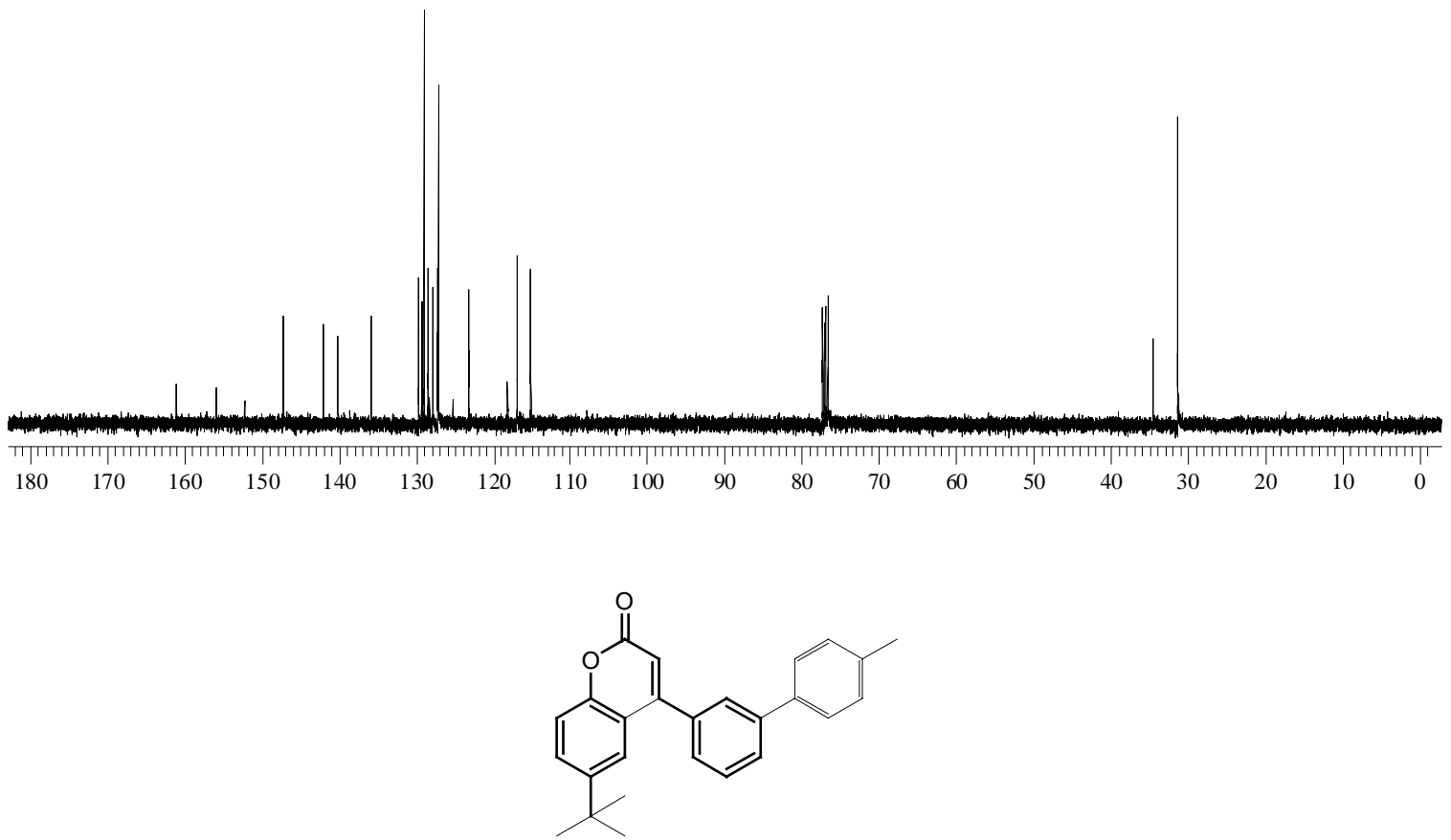

6-tert-Butyl-4-(3-p-tolylphenyl)-2H-chromen-2-one (3q). ${ }^{1} \mathrm{H}$ NMR $\left(\mathrm{CDCl}_{3}\right): \delta 7.75(\mathrm{~d}$, $\left.1 \mathrm{H}, P h \mathrm{PhCH}_{3}, J=7.9 \mathrm{~Hz}\right) ; 7.69\left(\mathrm{~s}, 1 \mathrm{H}, \mathrm{PhPhCH}_{3}\right) ; 7.60-7.64\left(\mathrm{~m}, 2 \mathrm{H},{ }^{t} \mathrm{Bu} P h, 1 \mathrm{H}\right.$; $\left.P h \mathrm{PhCH}_{3}, 1 \mathrm{H}\right) ; 7.58$ (d, $\left.1 \mathrm{H},{ }^{t} \mathrm{Bu} P h, J=1.8 \mathrm{~Hz}\right) ; 7.54$ (d, 2H, $\left.P h \mathrm{CH}_{3}, J=8.0 \mathrm{~Hz}\right) ; 7.43$ (d, $\left.1 \mathrm{H}, P h \mathrm{PhCH}_{3}, J=7.6 \mathrm{~Hz}\right) ; 7.37\left(\mathrm{~d}, 1 \mathrm{H},{ }^{t} \mathrm{Bu} P h, J=8.8 \mathrm{~Hz}\right) ; 7.29\left(\mathrm{~d}, 2 \mathrm{H}, P h \mathrm{CH}_{3}, J=8.0\right.$ $\mathrm{Hz}) ; 6.43$ (s, 1H, CHCO); 2.41 (s, 3H, $\left.\mathrm{PhCH}_{3}\right) ; 1.29$ (s, 9H, $\left.{ }^{t} \mathrm{Bu}\right) .{ }^{13} \mathrm{C}\left\{{ }^{1} \mathrm{H}\right\} \mathrm{NMR}: \delta 161.1$ (CO), $\mathrm{Ph}$ ring and $\mathrm{C}=\mathrm{C}(155.9,152.2,147.2,141.8,137.7,137.1,135.8,129.7,129.5$, 129.2, 128.2, 126.91, 126.87, 123.2, 118.2, 116.9, 115.0), ${ }^{t} \mathrm{Bu}(34.6,31.3), 21.1\left(\mathrm{PhCH}_{3}\right)$. $v_{\max }(\mathrm{NaCl}) / \mathrm{cm}^{-1}: 3058,1720,1616,1568,1481,1369,1184,1130$. HR-MS: $\mathrm{C}_{26} \mathrm{H}_{25} \mathrm{O}_{2}$ Calcd. 369.1855 (M+1); found: 369.1856. 

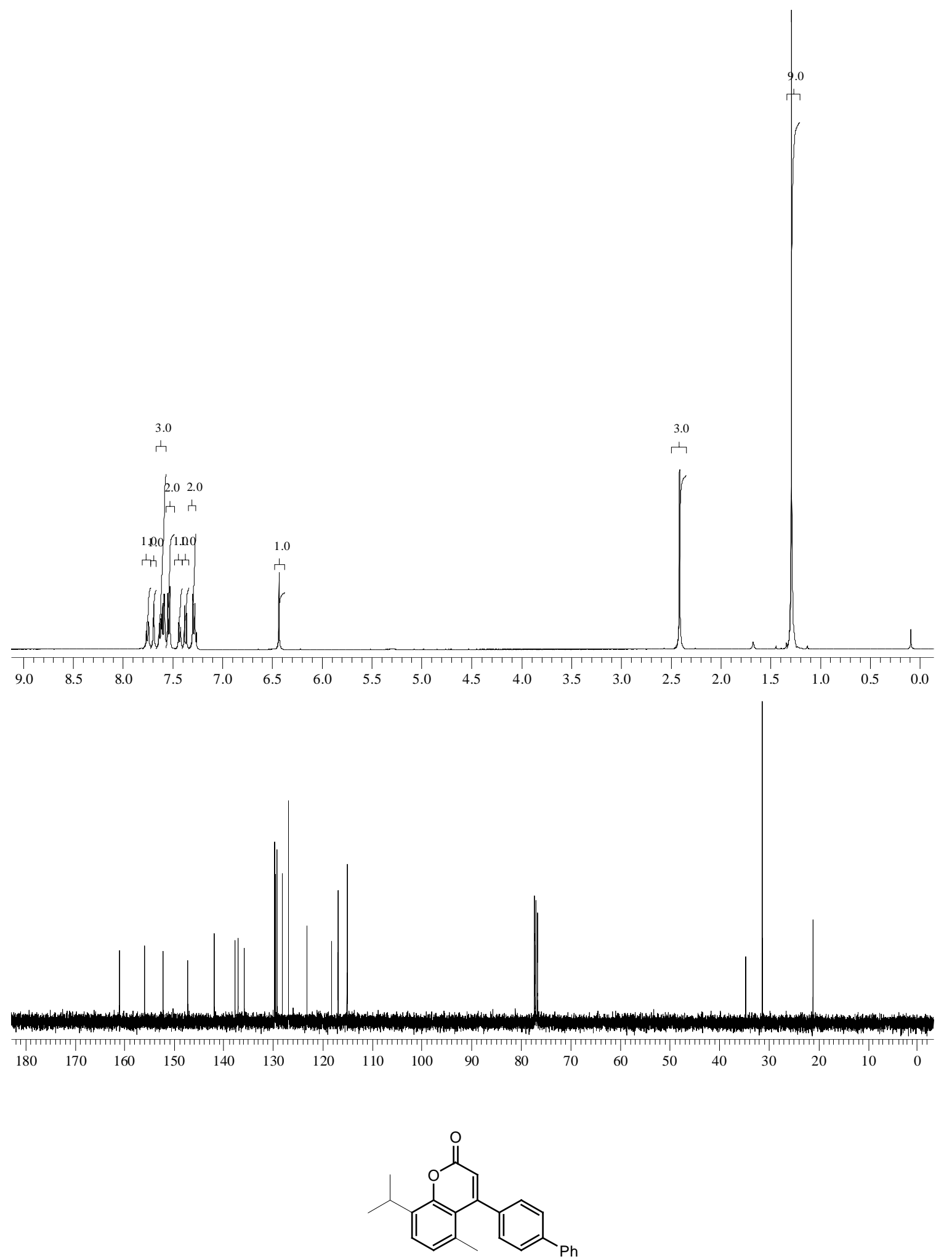

4-(4-Biphenyl)-8-isopropyl-5-methyl-2H-chromen-2-one (3r). ${ }^{1} \mathrm{H}$ NMR $\left(\mathrm{CDCl}_{3}\right)$ : $\delta$ $7.69(\mathrm{~d}, 2 \mathrm{H}, P h \mathrm{PhC}=\mathrm{CH}, J=7.6 \mathrm{~Hz}) ; 7.67(\mathrm{~d}, 2 \mathrm{H}, \mathrm{Ph} P h \mathrm{C}=\mathrm{CH}, J=8.2 \mathrm{~Hz}) ; 7.49(\mathrm{t}, 2 \mathrm{H}$, 
$P h \mathrm{PhC}=\mathrm{CH}, J=7.3 \mathrm{~Hz}) ; 7.36-7.42(\mathrm{~m}, 4 \mathrm{H}, \mathrm{Ph} P h \mathrm{C}=\mathrm{CH}, 2 \mathrm{H} ; P h \mathrm{PhC}=\mathrm{CH}, 1 \mathrm{H} ; \mathrm{PhO}, 1 \mathrm{H})$; 7.01 (d, 1H, PhO, J = $7.9 \mathrm{~Hz}) ; 6.31$ (s, 1H, CHCO); 3.66-3.73 (m, 1H, $\left.\mathrm{CHCH}_{3}\right) ; 1.88$ (s, $\left.3 \mathrm{H}, \mathrm{PhCH}_{3}\right) ; 1.33\left(\mathrm{~d}, 6 \mathrm{H}, \mathrm{CHCH}_{3}, J=6.7 \mathrm{~Hz}\right) .{ }^{13} \mathrm{C}\left\{{ }^{1} \mathrm{H}\right\}$ NMR: $\delta 160.3(\mathrm{CO})$, Ph ring and $\mathrm{C}=\mathrm{C}(156.9,152.1,141.5,140.0,138.7,134.8,134.6,128.9,128.4,128.0,127.8,127.7$, 127.1, 127.0, 117.6, 116.9), $\mathrm{CHCH}_{3}(26.5,22.7), 23.7\left(\mathrm{PhCH}_{3}\right) . v_{\max }(\mathrm{NaCl}) / \mathrm{cm}^{-1}: 3055$, 1720, 1614, 1580, 1487, 1447, 1415, 1385, 1346, 1196, 1051. HR-MS: $\mathrm{C}_{25} \mathrm{H}_{23} \mathrm{O}_{2}$ Calcd. $355.1698(\mathrm{M}+\mathrm{H})$; found: 355.1683 .

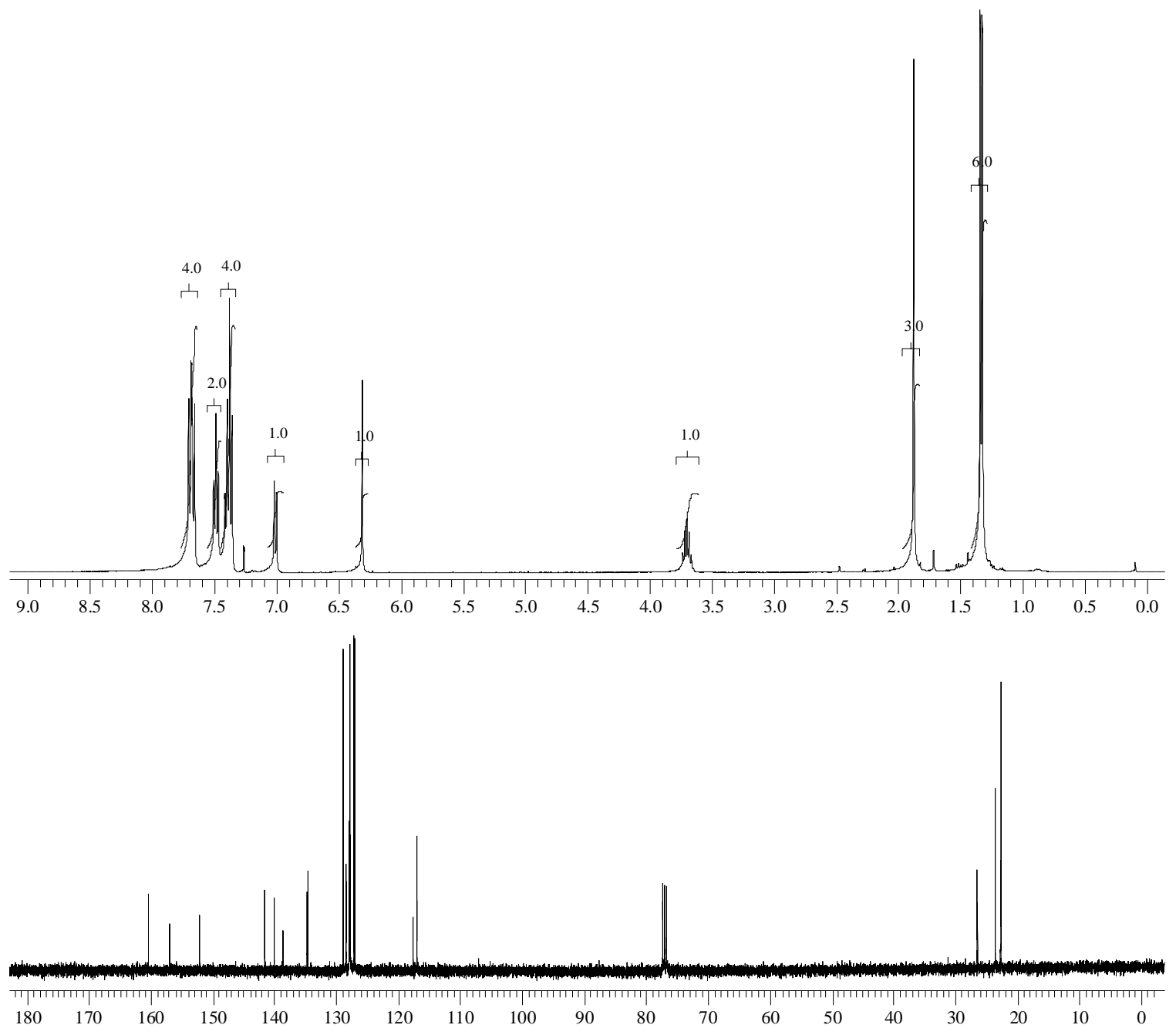




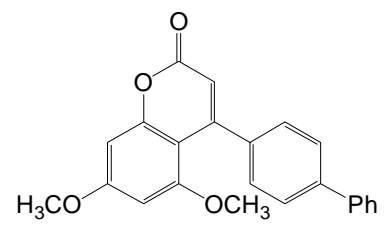

4-(4-Biphenyl)-5,7-dimethoxy-2H-chromen-2-one (3s). ${ }^{1} \mathrm{H}$ NMR $\left(\mathrm{CDCl}_{3}\right): \delta 7.67$ (d, $2 \mathrm{H}, P h \mathrm{PhC}, J=7.3 \mathrm{~Hz}) ; 7.62$ (d, 2H, PhPhC, $J=8.0 \mathrm{~Hz}) ; 7.48$ (t, 2H,PhPhC, $J=7.3$ $\mathrm{Hz}) ; 7.38$ (t, 1H, PhPhC, J = 7.3 Hz); 7.35 (d, 2H, PhPhC, $J=8.0 \mathrm{~Hz}) ; 6.54$ (d, 1H, $\left.P h \mathrm{OCH}_{3}, J=2.4 \mathrm{~Hz}\right) ; 6.25$ (d, 1H, $\left.P h \mathrm{OCH}_{3}, J=2.4 \mathrm{~Hz}\right) ; 6.06$ (s, 1H, CHCO); 3.88 (s, $\left.3 \mathrm{H}, \mathrm{PhOCH}_{3}\right) ; 3.46\left(\mathrm{~s}, 3 \mathrm{H}, \mathrm{PhOCH}_{3}\right) .{ }^{13} \mathrm{C}\left\{{ }^{1} \mathrm{H}\right\} \mathrm{NMR}: \delta 160.8(\mathrm{CO}), \mathrm{Ph}$ ring and $\mathrm{C}=\mathrm{C}$ $(163.3,158.1,157.1,155.3,140.6,138.6,128.8,127.7,127.5,127.0,125.9,112.6,103.4$, 95.7, 93.5), $\mathrm{PhOCH}_{3}(55.7,55.3) . v_{\max }(\mathrm{NaCl}) / \mathrm{cm}^{-1}: 3055,1718,1616,1597,1493,1466$, 1420, 1354, 1224, 1207, 1159, 1113, 1053. HR-MS: $\mathrm{C}_{23} \mathrm{H}_{19} \mathrm{O}_{4}$ Calcd. $359.1283(\mathrm{M}+1)$; found: 359.1303 .

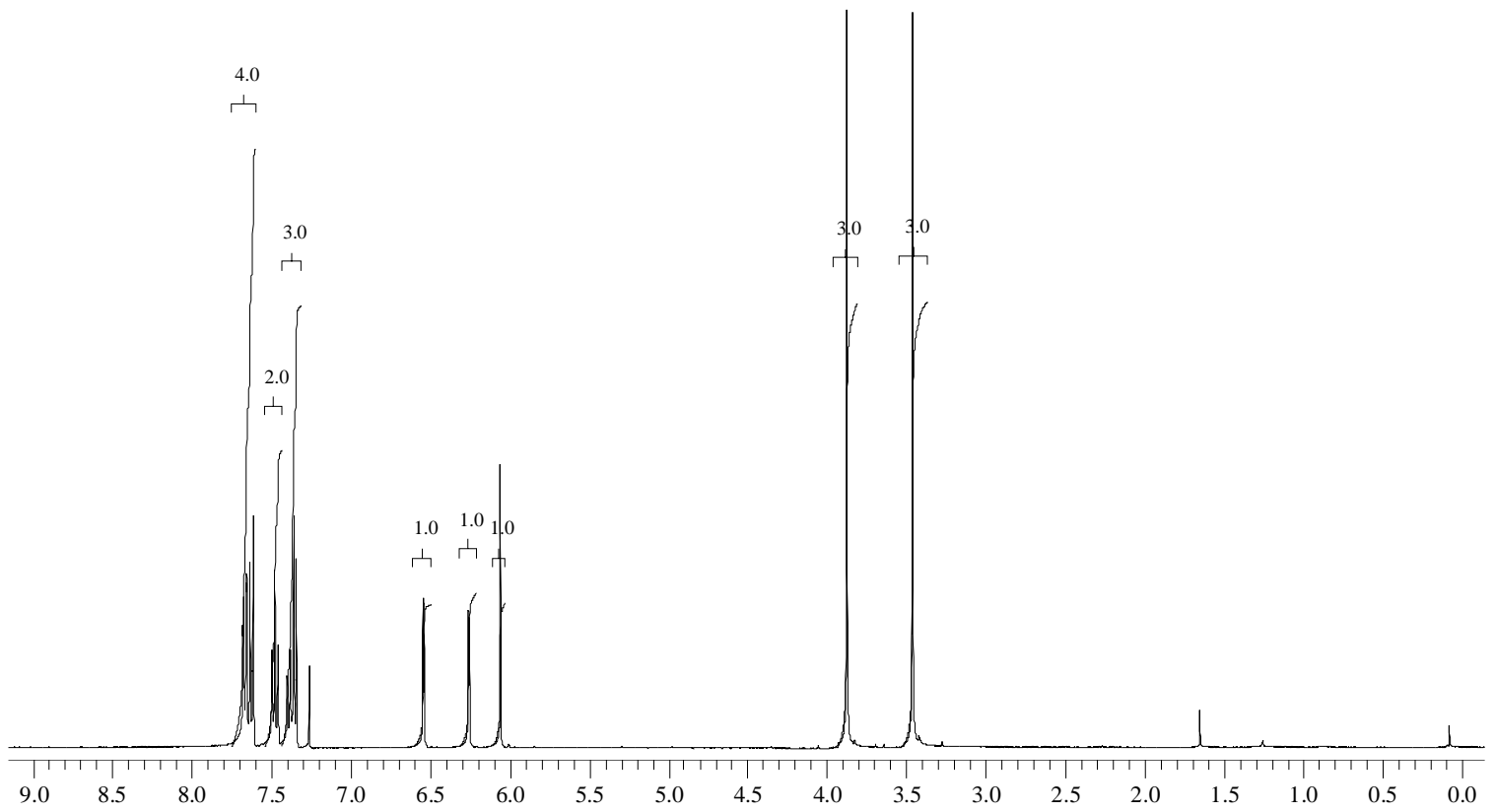



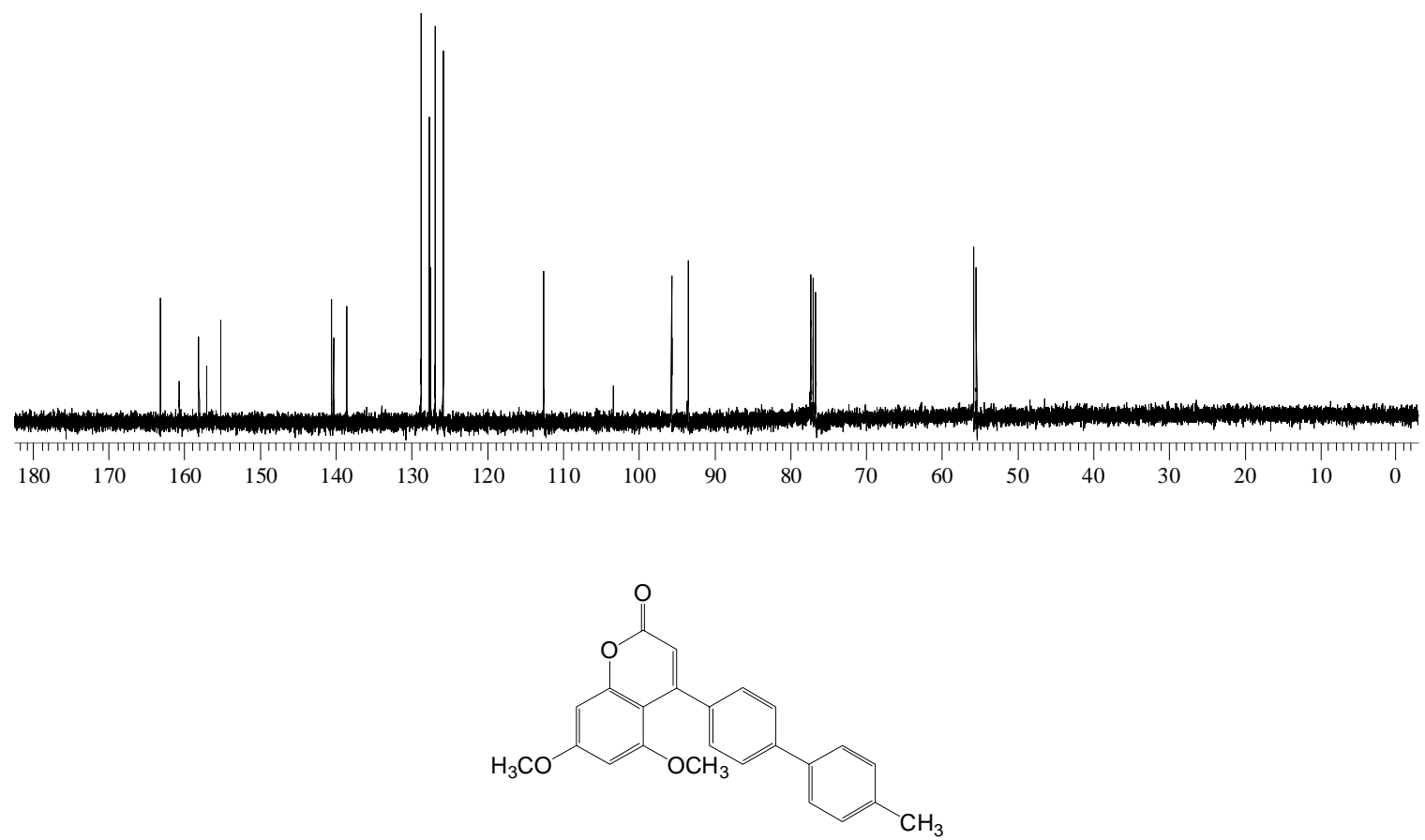

5,7-Dimethoxy-4-(4-p-tolylphenyl)-2H-chromen-2-one (3t). ${ }^{1} \mathrm{H}$ NMR $\left(\mathrm{CDCl}_{3}\right): \delta 7.61$

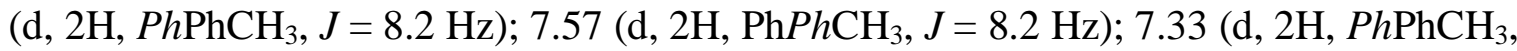
$J=8.2 \mathrm{~Hz}) ; 7.29\left(\mathrm{~d}, 2 \mathrm{H}, \mathrm{Ph} P h \mathrm{CH}_{3}, J=8.2 \mathrm{~Hz}\right) ; 6.54\left(\mathrm{~d}, 1 \mathrm{H}, P h \mathrm{OCH}_{3}, J=2.3 \mathrm{~Hz}\right) ; 6.25$ (d, $1 \mathrm{H}, P h \mathrm{OCH}_{3}, J=2.3 \mathrm{~Hz}$ ); 6.06 (s, $\left.1 \mathrm{H}, \mathrm{CHCO}\right) ; 3.88$ (s, $\left.3 \mathrm{H}, \mathrm{PhOCH}_{3}\right) ; 3.46$ (s, $3 \mathrm{H}$, PhOCH$H_{3}$ ); 2.42 (s, $\left.3 \mathrm{H}, \mathrm{PhCH}_{3}\right) .{ }^{13} \mathrm{C}\left\{{ }^{1} \mathrm{H}\right\}$ NMR: $\delta 160.9(\mathrm{CO})$, $\mathrm{Ph}$ ring and $\mathrm{C}=\mathrm{C}(163.3$, 158.2, 157.2, 155.4, 140.6, 138.4, 137.5, 137.4, 129.6, 127.7, 126.8, 125.7, 112.6, 103.5, 95.8, 93.5), $\mathrm{PhOCH}_{3}(55.8,55.4) . v_{\max }(\mathrm{NaCl}) / \mathrm{cm}^{-1}: 3055,1718,1614,1597,1498,1464$, 1354, 1225, 1208, 1159, 1113, 1053. HR-MS: $\mathrm{C}_{24} \mathrm{H}_{21} \mathrm{O}_{4}$ Calcd. $373.1440(\mathrm{M}+1)$; found: 373.1419 . 

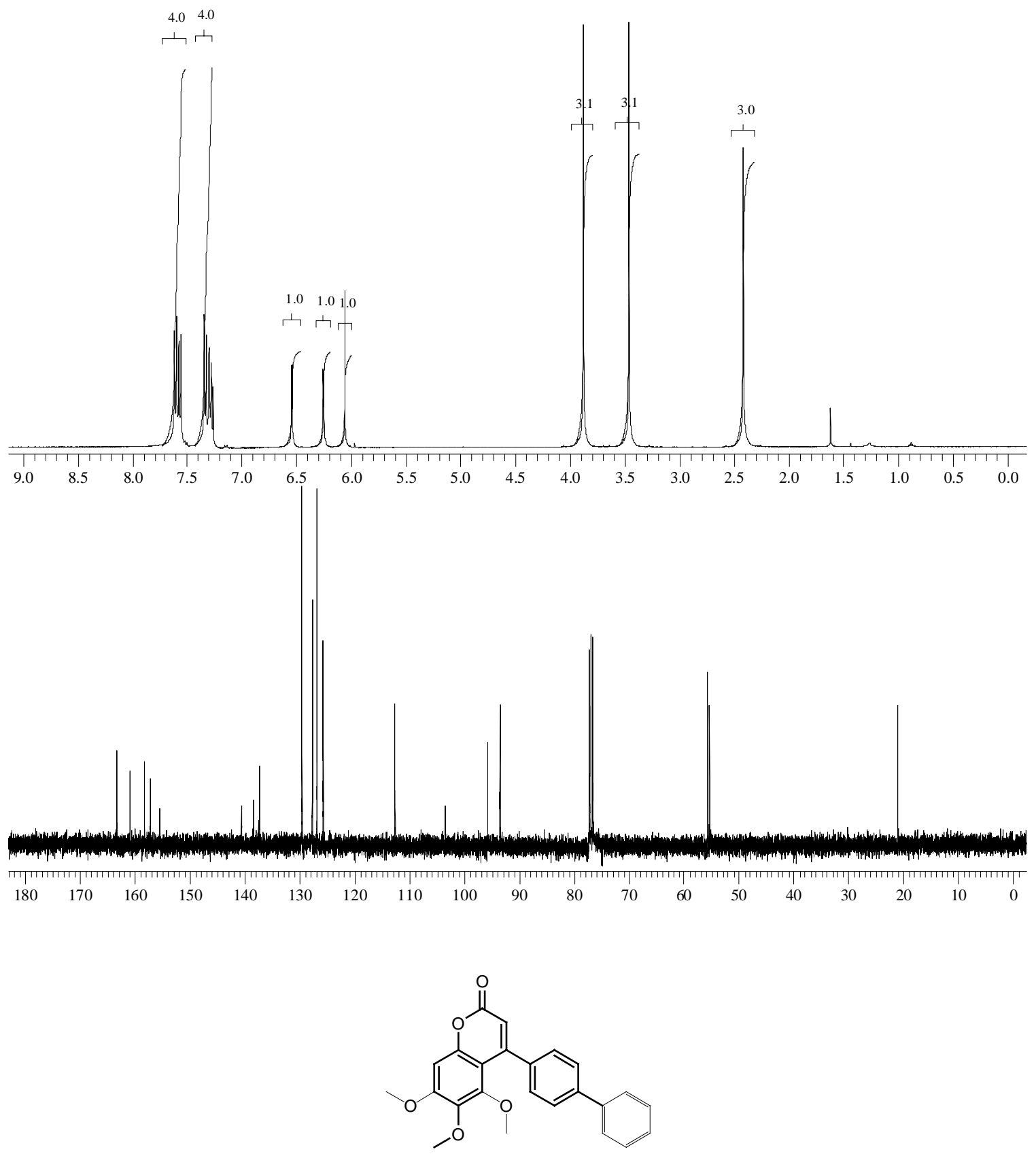

4-(4-Biphenyl)-5,6,7-trimethoxy-2H-chromen-2-one (3u). ${ }^{1} \mathrm{H}$ NMR $\left(\mathrm{CDCl}_{3}\right): \delta 7.67$ (d, $2 \mathrm{H}, P h \mathrm{PhC}, J=7.3 \mathrm{~Hz}) ; 7.66(\mathrm{~d}, 2 \mathrm{H}, \mathrm{Ph} P h \mathrm{C}, J=8.2 \mathrm{~Hz}) ; 7.48(\mathrm{t}, 2 \mathrm{H}, P h \mathrm{PhC}, J=7.3$ $\mathrm{Hz}$ ); 7.41 (d, 2H, $\mathrm{PhPhC}, J=8.2 \mathrm{~Hz}) ; 7.38$ (t, 1H, $P h \mathrm{PhC}, J=7.3 \mathrm{~Hz}$ ); 6.74 (s, 1H, PhO); 6.12 (s, $1 \mathrm{H}, \mathrm{CHCO}$ ); 3.95 (s, $3 \mathrm{H}, \mathrm{PhOCH}_{3}$ ); 3.81 (s, 3H, $\mathrm{PhOCH}_{3}$ ); 3.31 (s, $3 \mathrm{H}, \mathrm{PhOCH}_{3}$ ). ${ }^{13} \mathrm{C}\left\{{ }^{1} \mathrm{H}\right\}$ NMR: $\delta 160.5(\mathrm{CO})$, Ph ring and $\mathrm{C}=\mathrm{C}(156.8,155.0,151.6,151.0,140.7,140.3$, 139.3, 137.8, 128.8, 127.7, 127.5, 126.9, 126.0, 114.0, 107.1, 96.2), $\mathrm{PhOCH}_{3}(61.02$, 
60.96, 56.2). $v_{\max }(\mathrm{NaCl}) / \mathrm{cm}^{-1}: 3055,1720,1603,1549,1489,1458,1408,1364,1200$, 1132, 1101, 1036. HR-MS: $\mathrm{C}_{24} \mathrm{H}_{21} \mathrm{O}_{5}$ Calcd. 389.1389 (M+1); found: 389.1374.
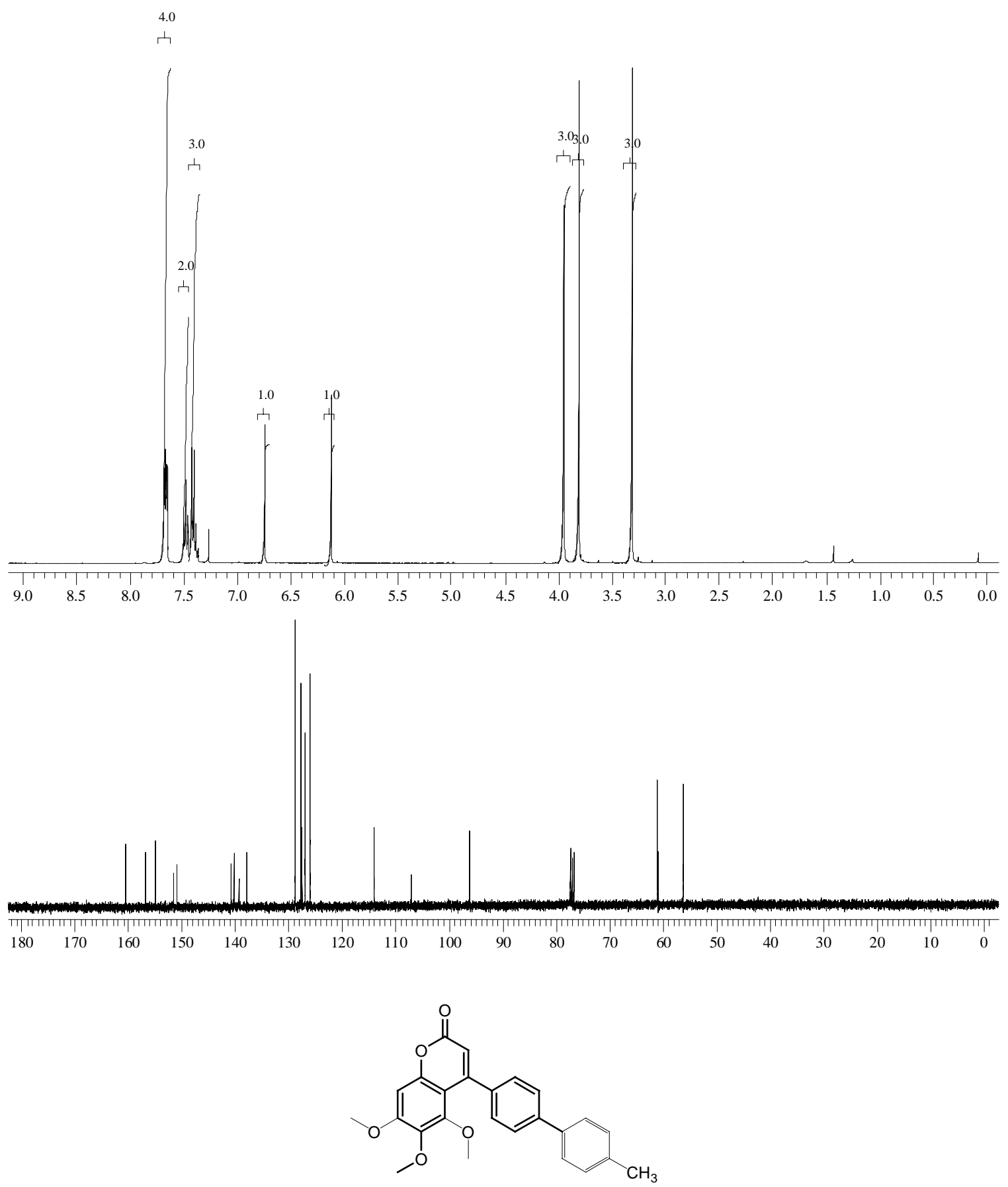

4-(4-p-Tolylphenyl)-5,6,7-trimethoxy-2H-chromen-2-one (3v). ${ }^{1} \mathrm{H}$ NMR $\left(\mathrm{CDCl}_{3}\right): \delta$ $7.64\left(\mathrm{~d}, 2 \mathrm{H}, P h \mathrm{PhCH}_{3}, J=8.0 \mathrm{~Hz}\right) ; 7.57\left(\mathrm{~d}, 2 \mathrm{H}, P h \mathrm{CH}_{3}, J=7.9 \mathrm{~Hz}\right) ; 7.39(\mathrm{~d}, 2 \mathrm{H}$, $\left.P h \mathrm{PhCH}_{3}, J=8.0 \mathrm{~Hz}\right) ; 7.28\left(\mathrm{~d}, 2 \mathrm{H}, P h \mathrm{CH}_{3}, J=7.9 \mathrm{~Hz}\right) ; 6.73(\mathrm{~s}, 1 \mathrm{H}, \mathrm{PhO}) ; 6.11(\mathrm{~s}, 1 \mathrm{H}$, $\mathrm{CHCO}$ ); 3.94 (s, $3 \mathrm{H}, \mathrm{PhOCH}_{3}$ ); 3.81 (s, $3 \mathrm{H}, \mathrm{PhOCH}_{3}$ ); 3.31 (s, $\left.3 \mathrm{H}, \mathrm{PhOCH}_{3}\right) ; 2.41$ (s, 
$\left.3 \mathrm{H}, \mathrm{PhCH} H_{3}\right) .{ }^{13} \mathrm{C}\left\{{ }^{1} \mathrm{H}\right\}$ NMR: $\delta 160.6(\mathrm{CO}), \mathrm{Ph}$ ring and $\mathrm{C}=\mathrm{C}(156.8,155.1,151.7,151.0$, 140.7, 139.3, 137.5, 137.4, 129.5, 127.7, 126.8, 125.8, 114.0, 107.1, 96.2), $\mathrm{PhOCH}_{3}$ (61.02, 60.96, 56.2), $21.1\left(\mathrm{PhCH}_{3}\right) . v_{\max }(\mathrm{NaCl}) / \mathrm{cm}^{-1}: 3061,1718,1605,1491,1460$, 1408, 1364, 1200, 1101，1018. HR-MS: $\mathrm{C}_{25} \mathrm{H}_{23} \mathrm{O}_{5}$ Calcd. $403.1545(\mathrm{M}+1)$; found: 403.1536.

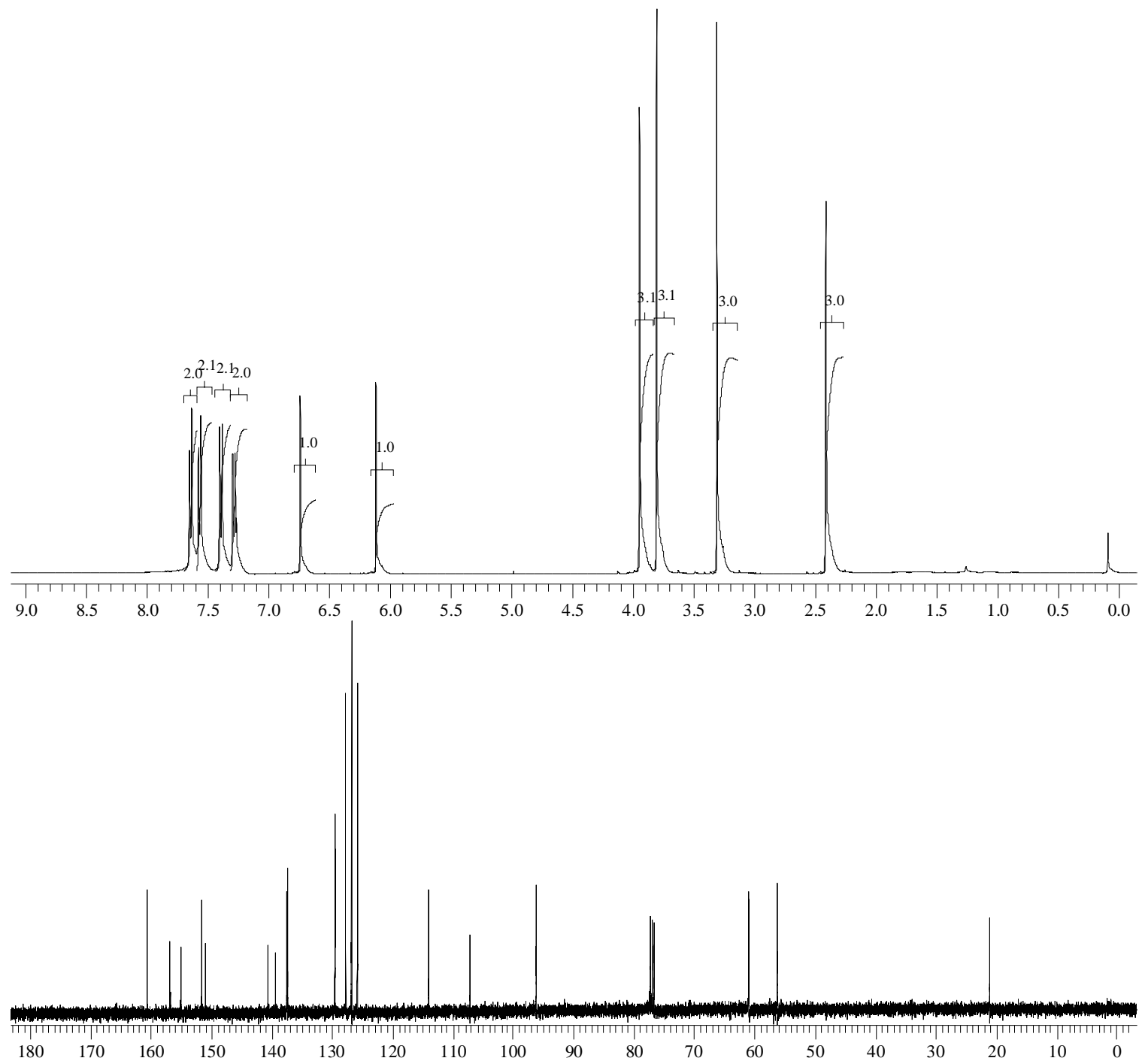




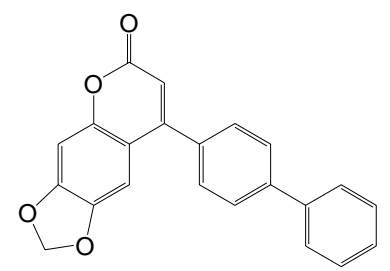

8-(4-Biphenyl)-6H-[1,3]dioxolo[4,5-g]chromen-6-one (3w). ${ }^{1} \mathrm{H}$ NMR $\left(\mathrm{CDCl}_{3}\right): \delta 7.73$ $(\mathrm{d}, 2 \mathrm{H}, P h \mathrm{PhC}, J=7.3 \mathrm{~Hz}) ; 7.65$ (d, 2H, PhPhC, $J=8.0 \mathrm{~Hz}$ ); 7.49 (t, 2H, $P h \mathrm{PhC}, J=7.3$ $\mathrm{Hz}) ; 7.48(\mathrm{~d}, 2 \mathrm{H}, \mathrm{PhPhC}, J=8.0 \mathrm{~Hz}) ; 7.41$ (t, 1H, $P h \mathrm{PhC}, J=7.3 \mathrm{~Hz}) ; 6.91$ (s, 1H, PhO); $6.90(\mathrm{~s}, 1 \mathrm{H}, \mathrm{PhO}) ; 6.28$ (s, $1 \mathrm{H}, \mathrm{CHCO}) ; 6.04-6.06\left(\mathrm{~m}, 2 \mathrm{H}, \mathrm{OCH}_{2} \mathrm{O}\right) .{ }^{13} \mathrm{C}\left\{{ }^{1} \mathrm{H}\right\}$ NMR: $\delta$ $161.1(\mathrm{CO}), \mathrm{Ph}$ ring and $\mathrm{C}=\mathrm{C}(155.5,151.3,151.1,144.8,142.5,139.9,134.4,128.9$, $128.7,127.9,127.5,127.1,112.7,112.0,104.3,98.5), 102.3\left(\mathrm{OCH}_{2} \mathrm{O}\right) . v_{\max }(\mathrm{NaCl}) / \mathrm{cm}^{-1}$ : 3055, 1717, 1634, 1576, 1504, 1485, 1445, 1406, 1389, 1334, 1209, 1138, 1040. HR-MS: $\mathrm{C}_{22} \mathrm{H}_{15} \mathrm{O}_{4}$ Calcd. $343.0970(\mathrm{M}+1)$; found: 343.0945.

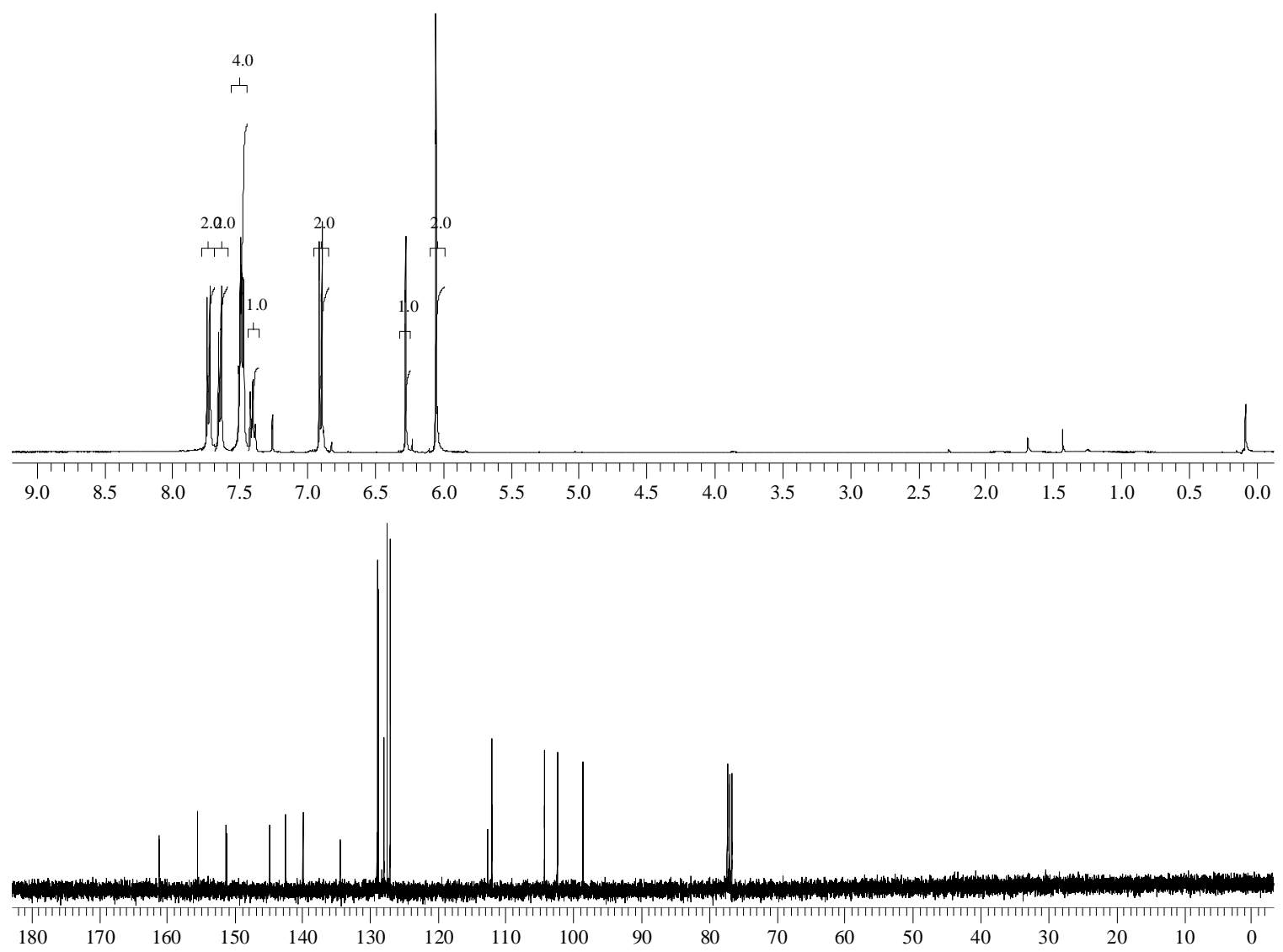




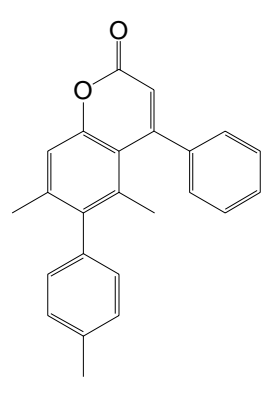

5,7-Dimethyl-4-phenyl-6-p-tolyl-2H-chromen-2-one (3x). ${ }^{1} \mathrm{H}$ NMR $\left(\mathrm{CDCl}_{3}\right): \delta$ 7.37$7.44(\mathrm{~m}, 3 \mathrm{H}, P h \mathrm{C}=\mathrm{CH}) ; 7.30(\mathrm{dd}, 2 \mathrm{H}, P h \mathrm{C}=\mathrm{CH}, J=7.6$ and $2.6 \mathrm{~Hz}) ; 7.20(\mathrm{~s}, 1 \mathrm{H}, P h \mathrm{O})$; 7.19 (d, 2H, $\left.P h \mathrm{CH}_{3}, J=7.9 \mathrm{~Hz}\right) ; 6.93\left(\mathrm{~d}, 2 \mathrm{H}, P h \mathrm{CH}_{3}, J=7.9 \mathrm{~Hz}\right) ; 6.24$ (s, $1 \mathrm{H}, \mathrm{CHCO}$ ); 2.37 (s, 3H, $\left.\mathrm{PhPhCH} H_{3}\right) ; 2.08$ (s, 3H, OPhCH$\left.H_{3}\right) ; 1.49$ (s, 3H, OPhCH$\left.H_{3}\right) .{ }^{13} \mathrm{C}\left\{{ }^{1} \mathrm{H}\right\}$ NMR: $\delta$ 160.6 (CO), $\mathrm{Ph}$ ring and $\mathrm{C}=\mathrm{C}(157.1,153.9,141.5,140.4,140.1,137.1,136.6,135.4$, 129.4, 129.0, 128.59, 128.57, 127.2, 116.7, 116.1, 116.0), $\mathrm{OPhCH}_{3}(21.8,21.7), 21.2$ $(\mathrm{OPhPhCH}) . v_{\max }(\mathrm{NaCl}) / \mathrm{cm}^{-1}: 3058,1720,1599,1541,1536,1211,1176,1069$. HRMS: $\mathrm{C}_{24} \mathrm{H}_{21} \mathrm{O}_{2}$ Calcd. $341.1542(\mathrm{M}+1)$; found: 341.1530.

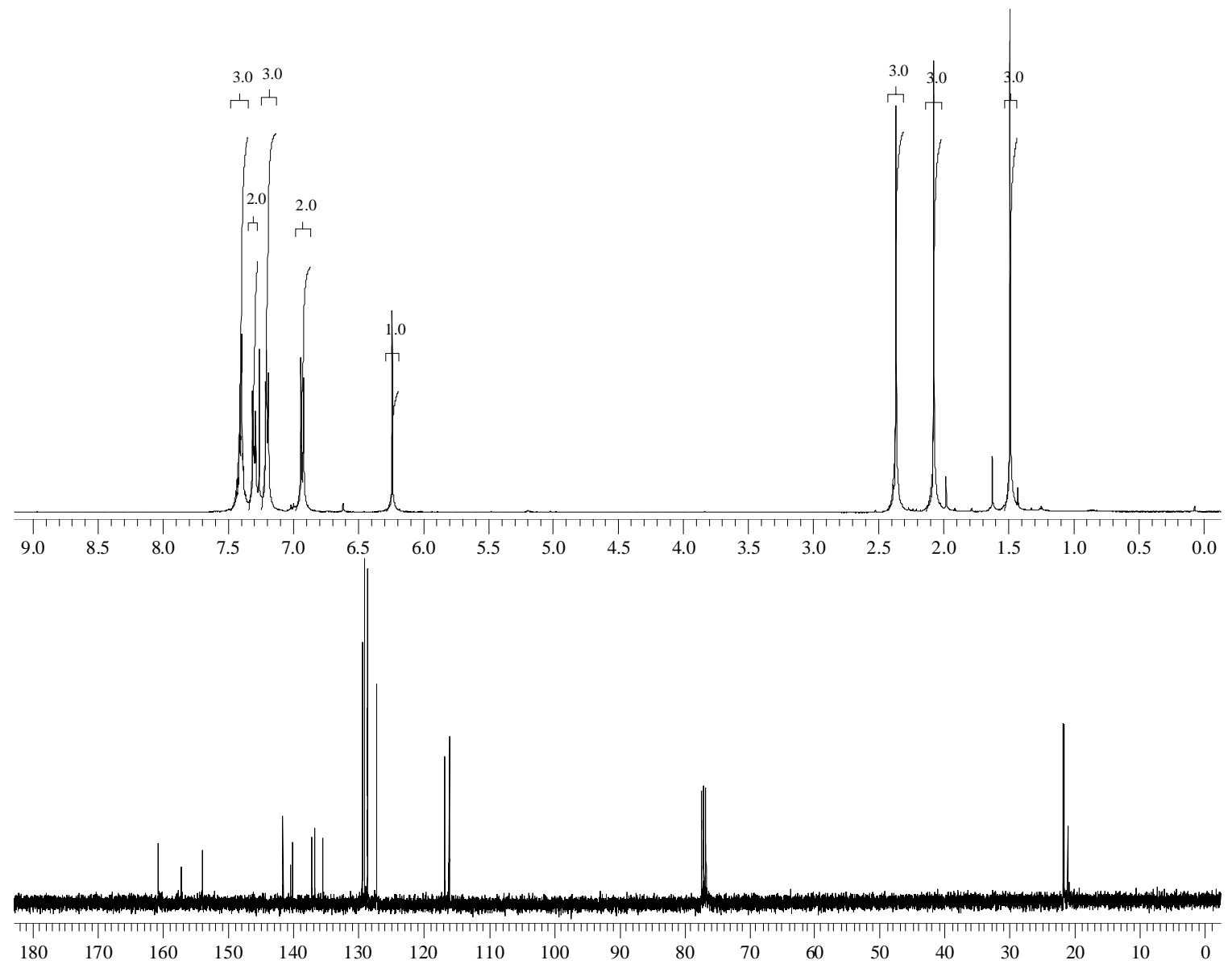




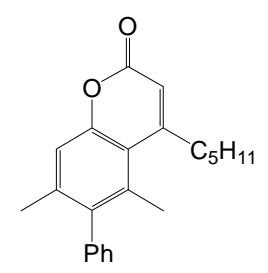

5,7-Dimethyl-4-pentyl-6-phenyl-2H-chromen-2-one (3y). ${ }^{1} \mathrm{H}$ NMR $\left(\mathrm{CDCl}_{3}\right): \delta 7.45(\mathrm{t}$, 2H, PhPhO, $J=7.6 \mathrm{~Hz}$ ); 7.37 (t, 1H, PhPhO, J= 7.6 Hz); 7.13 (s, 1H, PhPhO); 7.10 (d, $2 \mathrm{H}, P h \mathrm{PhO}, J=7.6 \mathrm{~Hz}$ ); 6.24 (s, 1H, CHCO); 2.90 (t, 2H, CH=CCH$H_{2}, J=7.9 \mathrm{~Hz}$ ); 2.33 (s, 3H, $\left.\mathrm{PhCH}_{3}\right) ; 2.02$ (s, 3H, $\left.\mathrm{PhCH}_{3}\right) ; 1.59-1.67$ (m, 2H, $\left.\mathrm{CH}=\mathrm{CCH}_{2} \mathrm{CH}_{2}\right) ; 1.30-1.42$ (m, $4 \mathrm{H}, \mathrm{CH}_{2} \mathrm{CH}_{2} \mathrm{CH}_{3}$ ); 0.90 (t, 3H, $\left.\mathrm{CH}_{2} \mathrm{CH}_{3}, J=7.3 \mathrm{~Hz}\right) .{ }^{13} \mathrm{C}\left\{{ }^{1} \mathrm{H}\right\}$ NMR: $\delta 160.9(\mathrm{CO}), \mathrm{Ph}$ ring and $\mathrm{C}=\mathrm{C}(158.8,154.0,140.7,140.6,140.3,134.1,129.2,128.8,127.1,117.0,116.6$, 115.0), $36.5\left(\mathrm{CCH}_{2}\right), 31.5\left(\mathrm{CCH}_{2} \mathrm{CH}_{2}\right), 29.6\left(\mathrm{CH}_{2} \mathrm{CH}_{2} \mathrm{CH}_{3}\right), 22.4\left(\mathrm{CH}_{2} \mathrm{CH}_{3}\right), 21.7$ $\left(\mathrm{PhCH}_{3}\right), 20.9\left(\mathrm{PhCH}_{3}\right), 13.9\left(\mathrm{CH}_{2} \mathrm{CH}_{3}\right) . v_{\max }(\mathrm{NaCl}) / \mathrm{cm}^{-1}: 3055,1718,1605,1546,1070$. HR-MS: $\mathrm{C}_{22} \mathrm{H}_{24} \mathrm{O}_{2} \mathrm{Na}$ Calcd. $343.1674(\mathrm{M}+\mathrm{Na})$; found: 343.1670 .

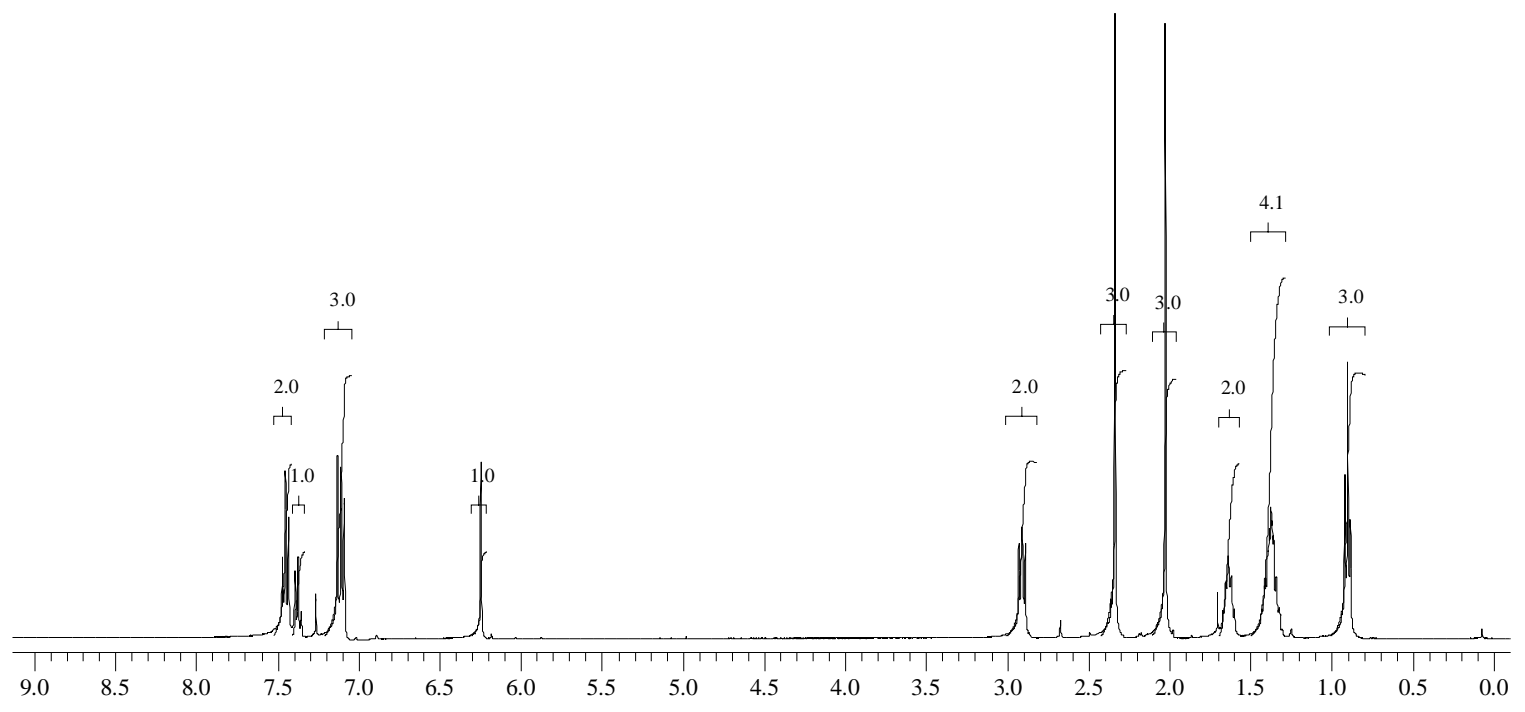



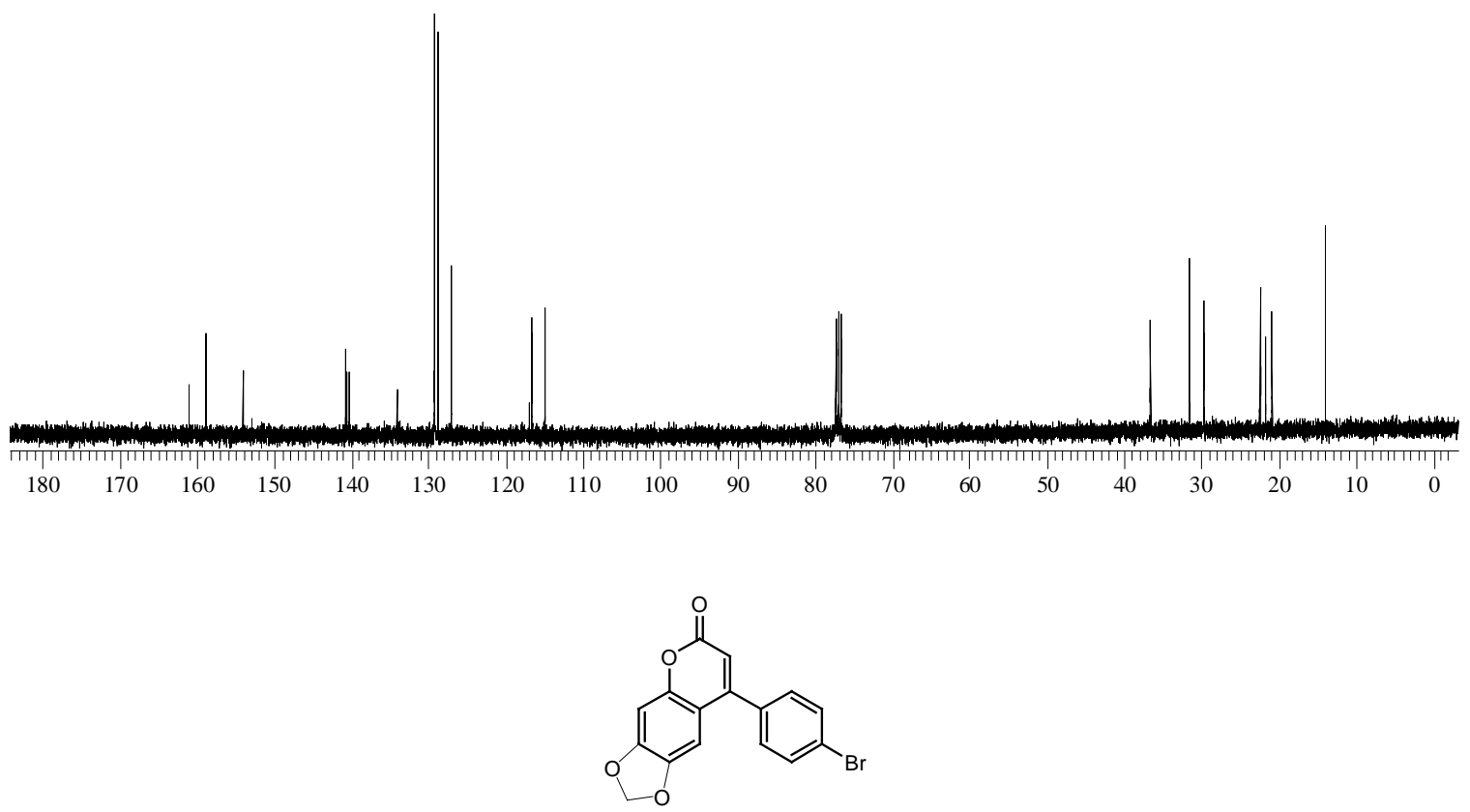

8-(4-Bromophenyl)-6H-[1,3]dioxolo[4,5-g]chromen-6-one. ${ }^{1} \mathrm{H}$ NMR $\left(\mathrm{CDCl}_{3}\right): \delta 7.59$ (d, 2H, PhBr, J= $8.8 \mathrm{~Hz}$ ); 7.23 (d, 2H, PhBr, J = $8.8 \mathrm{~Hz}$ ); 6.83 (s, 1H, PhO); 6.69 (s, 1H, $\mathrm{PhO}$ ); 6.15 (s, 1H, CHCO); 5.99 (s, 2H, $\left.\mathrm{OCH}_{2} \mathrm{O}\right) .{ }^{13} \mathrm{C}\left\{{ }^{1} \mathrm{H}\right\}$ NMR: $\delta 159.9(\mathrm{CO}), \mathrm{Ph}$ ring and $\mathrm{C}=\mathrm{C}(153.6,150.4,150.3,144.0,133.5,131.2,128.8,123.0,111.4,111.3,103.0$, 97.7), $101.4\left(\mathrm{OCH}_{2} \mathrm{O}\right) . v_{\max }(\mathrm{NaCl}) / \mathrm{cm}^{-1}: 3055,1724,1633,1575,1559,1504,1485$, 1447, 1375, 1136, 1040. HR-MS: $\mathrm{C}_{16} \mathrm{H}_{10} \mathrm{O}_{4} \mathrm{Br}$ Calcd. $344.9762(\mathrm{M}+\mathrm{H})$; found: 344.9750.

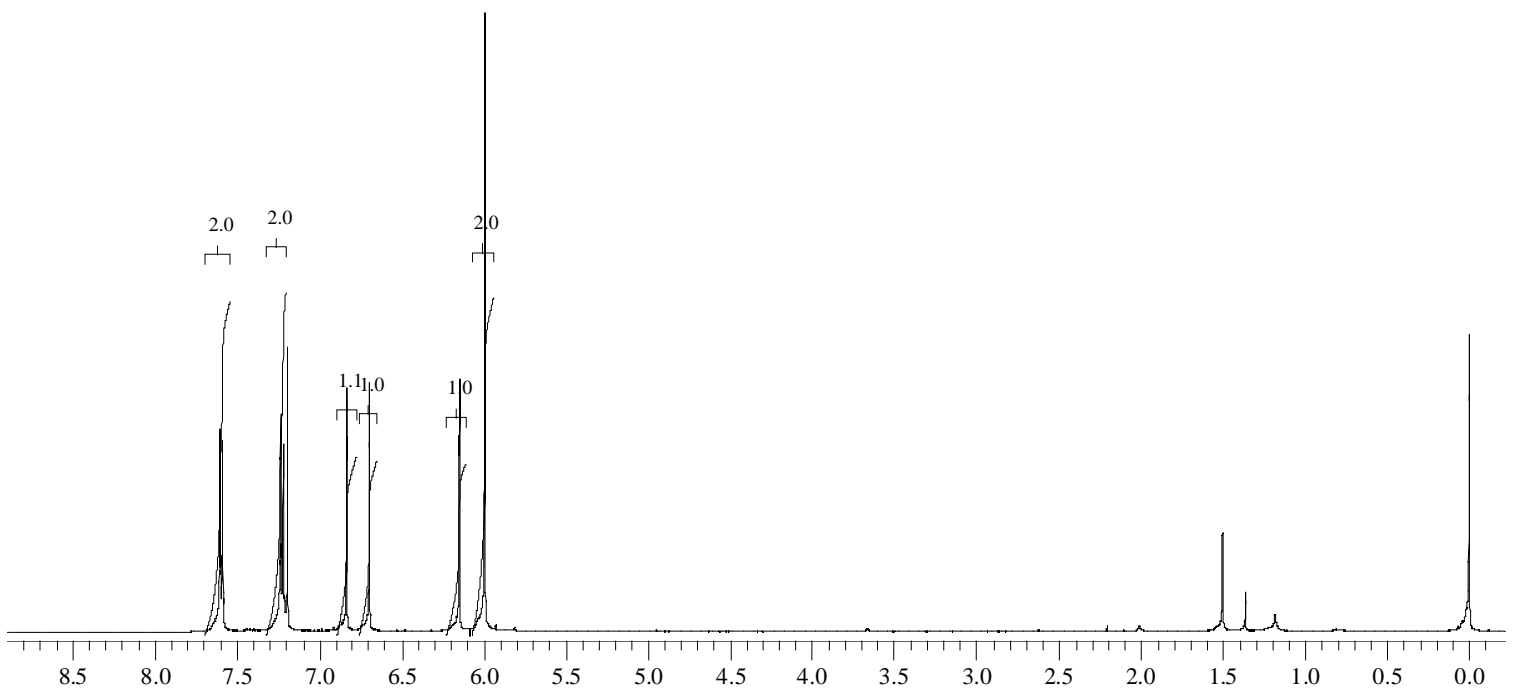




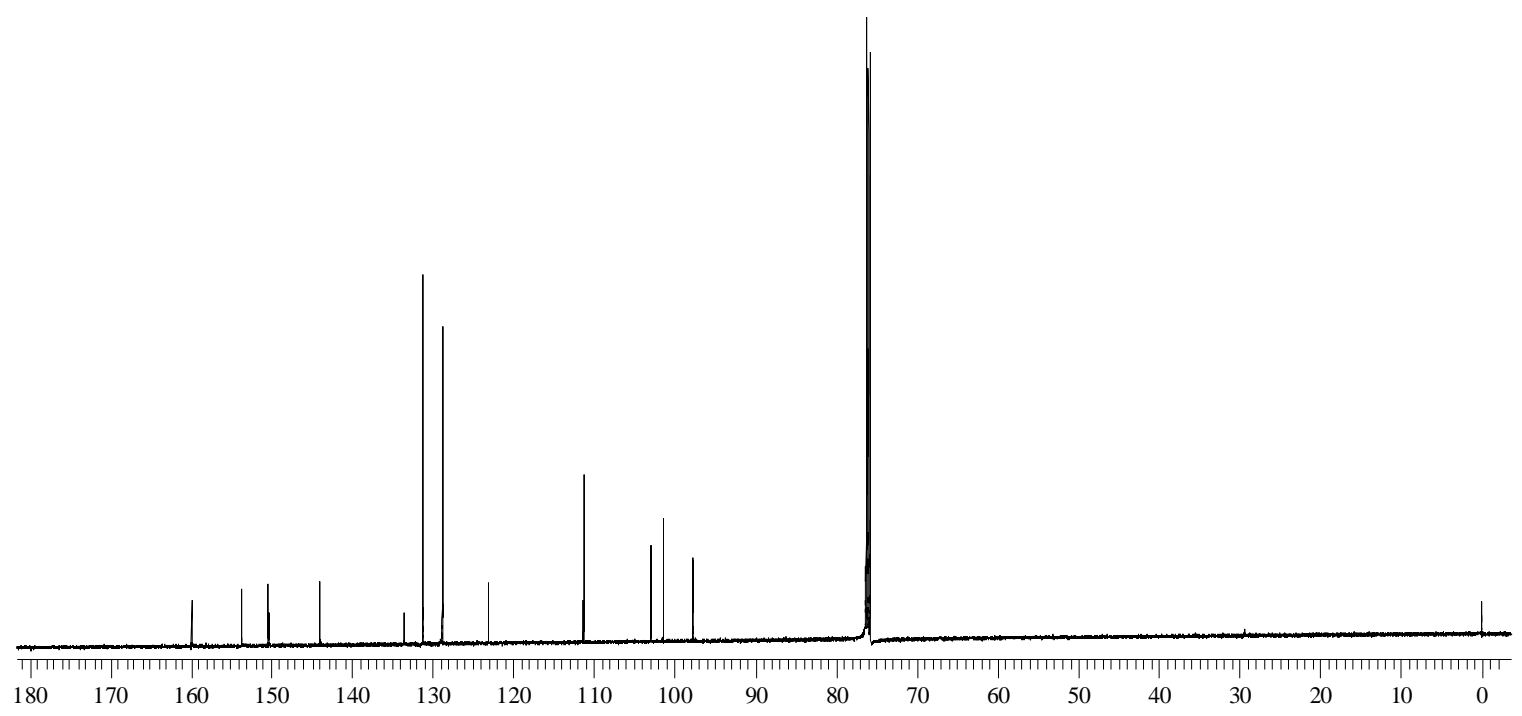

${ }^{1}$ (a) Sheeham, J. C.; Hess, G. P.; J. Am. Chem. Soc., 1955, 77, 1068. (b) Neises, B.; Steglich, W.; Angew. Chem., Int. Ed. Engl., 1978, 17, 523.

2 (a) Jia, C.; Piao, D.; Oyamada, J.; Lu, W.; Kitamura, T.; Fujiwara, Y. Science, 2000, 287, 1992-1995. (b) Jia, C.; Piao, D.; Kitamura, T.; Fujiwara, Y. J. Org. Chem. 2000, 65, 7516-7522. 